
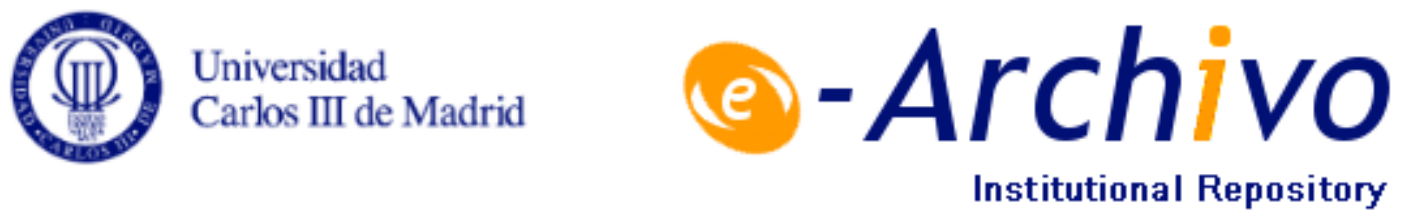

This is a postprint version of the following published document:

Vázquez, J. Montalvo et al. (2012)."Signal processing, management and monitoring in transmission networks", in Optical Transmission: the FP7 BONE Project Experience, pp.53-122. Available in http://dx.doi.org/10.1007/978-94-007-1767-1_2

(C) 2012 Springer 


\title{
Signal Processing, Management and Monitoring in Transmission Networks
}

\author{
Carmen Vázquez, Julio Montalvo, Jawaad Ahmed, David Bolt, Christophe \\ Caucheteur, Gerald Franzl, Philippe Gravey, David Larrabeiti, Jose A. Lazaro, \\ Tatiana Loukina, Veronique Moeyaert, Josep Prat, Lena Wosinska, \\ and Kivilcim Yüksel
}

C. Vázquez ，J. Montalvo ，D. Larrabeiti

Department of Electronic Engineering, Universidad Carlos III de Madrid, 28911 Madrid, Spain e-mail: cvazquez@ing.uc3.es; julio.montalvo@uc3m.es; dlarra@ing.uc3.es

\section{J. Ahmed}

The Royal Institute of Technology (KTH), Electrum 229, 16440 Kista, Stockholm, Sweden e-mail: jawwad@kth.se

D. Bolt

College of Engineering, Swansea University, Singleton Park, SA2 8PP Swansea,

West Glamorgan, UK

e-mail: 393729@Swansea.ac.uk

C. Caucheteur , V. Moeyaert , K. Yüksel

Faculté Polytechnique, Electromagnetism and Telecommunication Unit, Université de Mons, 31, Boulevard Dolez, Mons 7000, Belgium

e-mail: christophe.caucheteur@umons.ac.be; Veronique.MOEYAERT@umons.ac.be;

kivilcim.yuksel@umons.ac.be

G. Franzl

Institute of Telecommunications, Vienna University of Technology, Favoritenstraße 9/389

A-1040, Vienna, Austria

e-mail: Gerald.Franzl@tuwien.ac.at

P. Gravey $\bullet$ T. Loukina

Telecom Bretagne, Technop^ole Brest-Iroise, CS, 83818-29238 Brest Cedex 3, France

e-mail: philippe.gravey@telecom-bretagne.eu; Tatiana.loukina@telecom-bretagne.eu

\section{J.A. Lazaro}

BarcelonaTech. Department of Signal Theory and Communication, Universitat Politècnica de Catalunya, 08034 Barcelona, Spain

e-mail: jose.lazaro@tsc.upc.edu

J. Prat

Department of Signal Theory and Communication, Polytechnic University of Catalonia, 08034 Barcelona, Spain

e-mail: jprat@tsc.upc.edu

\section{Wosinska}

The Royal Institute of Technology (KTH), Electrum 229, 16440 Kista, Stockholm, Sweden e-mail:wosinska@kth.se 


\section{Introduction}

For transparent optical networks, it is important to support the demanded signal quality of physical paths from source to destination nodes, in response to a given call request. Due to the lack of regeneration in current optical networks, optical signal impairments accumulate along paths. With channel bit rates of $10 \mathrm{Gbit} / \mathrm{s}$ or higher, linear and non-linear fibre impairments become prominent factors affecting the signal quality. Thus, new techniques in both physical layer and network layer are necessary for mitigating impairments to accommodate high-speed traffic (Tomkos et al. 2002).

On the other hand, the flexibility of modern networks with dynamic and distributed management, where lightpaths can be dynamically and automatically assigned end-to-end, increases the risk of changing path conditions that affect signal quality and consequently quality of service (QoS) over the lifetime of a connection as well as for subsequent connection set-up requests (on-demand routing and wavelength assignment - RWA). For traditional connection provisioning an ideal physical layer, ignoring transmission impairments (Chlamtac et al. 1992) could be assumed because the implicit per hop regeneration (optics-to-electronics-to-optics conversion - $\mathrm{O} / \mathrm{E} / \mathrm{O}$ ) compensated signal impairments hop-by-hop in a rather static manner (section commissioning). For end-to-end all-optically switched connections using photonic cross-connect switches (PXC), the conditions change and common practice is not applicable. Anyhow, intelligent connection provisioning is an important traffic engineering problem, and minimising operational cost as well as efficiently utilising network resources is the main driver. In this context, it seems important to have flexible routing protocols that take into consideration the most relevant physical impairments, and are able to exchange messages with their values as part of the route information with others. This condition, if definitely used to calculate routing, will surely assure better success in data delivery over the network (Huang et al. 2005).

Irrespective of the control architecture chosen by an operator of an optical network, the included control plane (in charge of setting up and tearing down optical circuits; also known as lightpaths) needs to have a proper set of tools to satisfyingly deal with physical impairments. The most essential tools are: optical signal monitoring, signal processing and impairment compensation techniques (each all-optically and/or electrically).

With the help of these tools, it is possible to predict the QoT (quality of transmission) attainable for a lightpath at a given time (i.e. the latest network-wide monitoring instant), and the control plane can route requests across the network 
according to this information, keeping the circuit as much as possible in the optical layer. In this chapter, we introduce and use a specific signalling model, the IETF GMPLS framework, to illustrate how impairment information and impairment compensation tools can be dealt with under a distributed control plane paradigm. The use of GMPLS obeys to simplicity of presentation of concepts and to the fact that ITU-T and OIF have adopted GMPLS signalling and routing protocols as valid implementation alternatives. However, the techniques for impairment management described here can be easily applied to any other optical network control architecture under standardisation. Optical burst and packet switching are thus not considered, as these are currently not under standardisation. They actually demand sophisticated signalling latency independent and thus less accurate, but in response more robust, approaches.

Real-time monitoring of physical impairments related to transmission media properties such as chromatic dispersion, attenuation, and non-linear effects at different wavelengths can be achieved by different optical monitoring techniques, which have greatly evolved in the last years. Even optical compensation techniques that alleviate the effect of impairments are important to predict the signal quality along a lightpath. Having in mind that, in a wavelength-routed optical network spanning a large geographical area, optical signals may traverse several long fibre segments and a number of different compensation devices, we recognise that an integration view of their effects should be taken into account for selecting a path (Teixeira et al. 2009). Impairment-aware routing and wavelength assignment (IA-RWA) algorithms considering TE information together with the locally introduced physical impairments and the availability of compensation devices through the network can be employed to compute a feasible and least-costly (optimal) lightpaths from sources to destinations. Impairment-aware on-demand routing is conceptually superior to shortest-path routing with maximum transparent distance constraint as well as exhaustive offline constraint-based routing algorithms (Pachnicke et al. 2009).

The basic ideas of general multi-protocol label switching (GMPLS), being the protocol framework intended to control dynamic circuit set-up and teardown in the next generation of optical transport networks, are reported in Sect. 2.2, along with the description of the main routing protocols, new tendencies and their operation procedures and flexibility in selecting routes for lightpaths. At the end of the section, some aspects about convergence between transport and access networks, mainly in terms of physical impairment monitoring and compensation, are discussed. Section 2.3 presents different optical monitoring techniques (including spectral and time domain techniques) and some dispersion compensation techniques. Approaches respecting constraints for end-to-end QoT control (related to changing physical properties along lightpaths) and different impairment-aware routing alter-natives are discussed in Sect. 2.4. Finally, summarising conclusions are outlined on Sect. 2.5. 


\section{Dynamic and Distributed Control in Transmission Networks}

\subsection{IP Networks and Circuits:IP-MPLS}

Nowadays, there are two clearly differentiated overlaid networks making up the backbone of wide area data networks: the packet-switched networks and the underlying electro-optical circuit-switched long distance transport networks. Today, IP is the dominating WAN packet-switching network technology in the world. Given its importance, there is a growing interest to design optical transport networks tailored for IP-based data communication. Conversely, packet networks have tried to incorporate features primarily available in circuit networks. This latter trend has made MPLS become a commodity on IP backbone networks: it enables to extend IP routers with circuit-oriented capabilities. We will start to review the basic ideas that led to MPLS in order to understand GMPLS (generalised MPLS), the protocol framework proposed by IETF intended to control circuit set-up and teardown in the next generation of optical transport networks.

IP is a network layer protocol (layer-3 in the OSI reference model) intended to provide end-to-end packet delivery between two end systems (usually hosts) connected to an IP network. To this end, hosts are assigned IP addresses, and the packet headers carry the source and destination addresses (32 bits in the case of IPv4 and 128 bits in IPv6). The forwarding of packets is performed by packet switches (routers) interconnected by links. Since IP has not a single predefined link layer technology to work upon a logical IP link, connecting two neighbouring routers can be realised by any digital communication service between them adapted by a suitable link layer. Today, Internet core routers are typically interconnected by point-to-point 2.5, 10 and $40 \mathrm{Gbit} / \mathrm{s}$ electro-optical channels (direct or circuit-switched), and IP packets are sent in different ways, such as Ethernet link-layer frames. Towards the edge of the network, links between IP nodes consist of one or more link layer sections spanning a greater variety of physical transmission media (usually radio, copper or fibre). Each link technology has its own link and medium access control protocols that are opaque to IP.

In IP, packet forwarding is basically driven by the packet's destination IP address and the router's routing table. The next hop selected for a packet is determined by the longest-matched network prefix in the routing table and usually by additional policy rules. This is a relatively complex operation that, in core routers, is expedited either by route lookup hardware (e.g. TCAM) or by SRAM forwarding caches. Each router constructs and maintains its own routing table and takes the forwarding decision autonomously, in a fully distributed way. The routing table is created on each router's CPU either by static configuration of routes or by a routing protocol. An IP network administrator needs to decide what routing protocol to use within its own domain, i.e. the IGP, and which routing protocol the domain's border routers need to use to exchange routes with other domains (also called autonomous 
systems - AS). This latter is the EGP which, in the case of the public Internet, is agreed to be the BGP. All routing tables are thus automatically populated by these two routing protocols.

From the two major families of IGP routing protocols, distance-vector and link state-based, derived recent link-state protocols which became predominant for their globally faster recovery from link failures. The most popular standard link state routing protocols are: OSPF and ISIS. With link-state protocols, each router monitors the state of the link to each neighbour and floods this information over the network either periodically or upon an event (e.g. a link-down event). Whenever a link-state advertisement is received by a router, the router reviews its routing table and updates the next-hop entry for each route's network prefix. The next hop is the IP address of the router on the shortest path to the destination according to the established metrics. If a link or router fails, the neighbouring routers react and convey the news to the rest of the network, causing an update of the network topology as seen by the routing process at each node.

There are a number of intrinsic limitations to the way IP networks operate that can actually be summarised into one: IP does not provide enough flexibility to support TE.

Firstly, since, as stated, the IP forwarding decision is distributed and only based on the IP address, all packet flows tend to take the shortest path, which is not always the right choice: Alternative longer paths may be underutilised and could be used to transport part of the total traffic. Available routing-based traffic balancing mechanisms (such as OSPF's equal-cost multipath or the use of a RTT metric) are not flexible enough and have side effects difficult to foresee and handle.

Secondly, it is not possible to implement fast-recovery protection mechanisms for traffic trunks. Due to the distributed nature of IP routing, recovery times are by far longer than the ones of optical networks, such as SDH or SONET, assuring a convergence time of less than $50 \mathrm{~ms}$ (Valenti et al. 2009). Depending on the size and complexity of the network topology, the recovery process of IP networks may take up to seconds before all routers converge and create new loop-free paths. This effect, which could be regarded unimportant in the past, today is an obstacle to realise the multi-service network concept, where the IP backbone is expected to carry voice and TV traffic trunks in a very reliable way (substituting legacy telecommunication services), as well as regular TCP/IP traffic. Hence, it is essential to have in IP networks the same capability known from SDH/SONET networks, i.e. the ability to set up primary and back-up paths for fast switchover in the event of a failure, as nowadays readily provided by Carrier Ethernet. To be effective, this should be made in such a way that the backup path is predetermined, insensitive to transitory routing instabilities, and its spare capacity needs to be reserved in advance in order to carry instantly all the diverted traffic.

Consequently, a mechanism to set up virtual circuits in an IP network for traffic balancing and path protection was deemed very useful. This functionality is enabled by MPLS. MPLS provides a way to create unidirectional virtual circuits, named LSP. A router enhanced with MPLS capability is known as a LSR. In MPLS, IP packets (or any other protocol data unit, PDU) are encapsulated into MPLS 


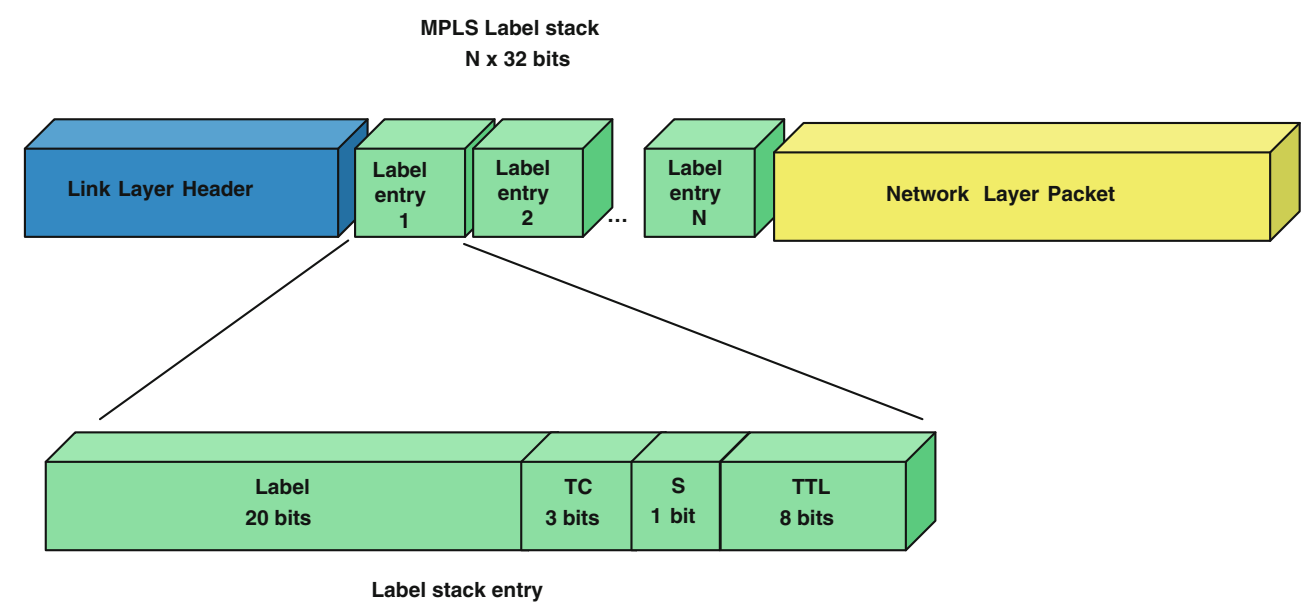

Label: Label \#

TC: Traffic Class of frame S:

Bottom of Stack. TTL: Time to

Label space can be per-interface or per-platform Usually live.

Value 1 for the innermost label stack entry Used to detect loops

Fig. 2.1 MPLS generic label encapsulation

frames at the ingress LSR and label-switched all the way through a pre-established LSP to the egress LSR, where the MPLS header is removed. MPLS headers are very simple (see Fig. 2.1) and so is the forwarding mechanism: given an input port and input label, a single lookup into the LFIB provides the output port, next hop and the operation to perform on the frame (label swap, pop, push or a combination). In fact, the original purpose of label switching was speeding up packet forwarding. This forwarding mechanism based on stackable 32-bit labels is a fixed aspect of MPLS. What is variable or open is the control plane, i.e. the protocols that determine the route and signal the set-up and teardown of LSPs.

The signalling protocol must create a binding between an FEC and a label. An FEC is an identifier for a specific set of packet flows that need to be forwarded in exactly the same way by the LSRs along the LSP created for that FEC; in other words, packets of the same FEC follow the same path(s) and receive identical QoS treatment. An LSP may be created:

- either by following the forwarding path determined by the routing protocol. In this case, the most commonly used protocol is the LDP. In this setting, LDP creates automatically LSPs from each node to every other network node along the shortest paths.

- Or over an arbitrarily specified path. This can be made either by manual configuration of all LSRs along a path or by signalling initiated by the LSP's ingress router using RSVP-TE, RFC3209. This is the signalling protocol commonly used 


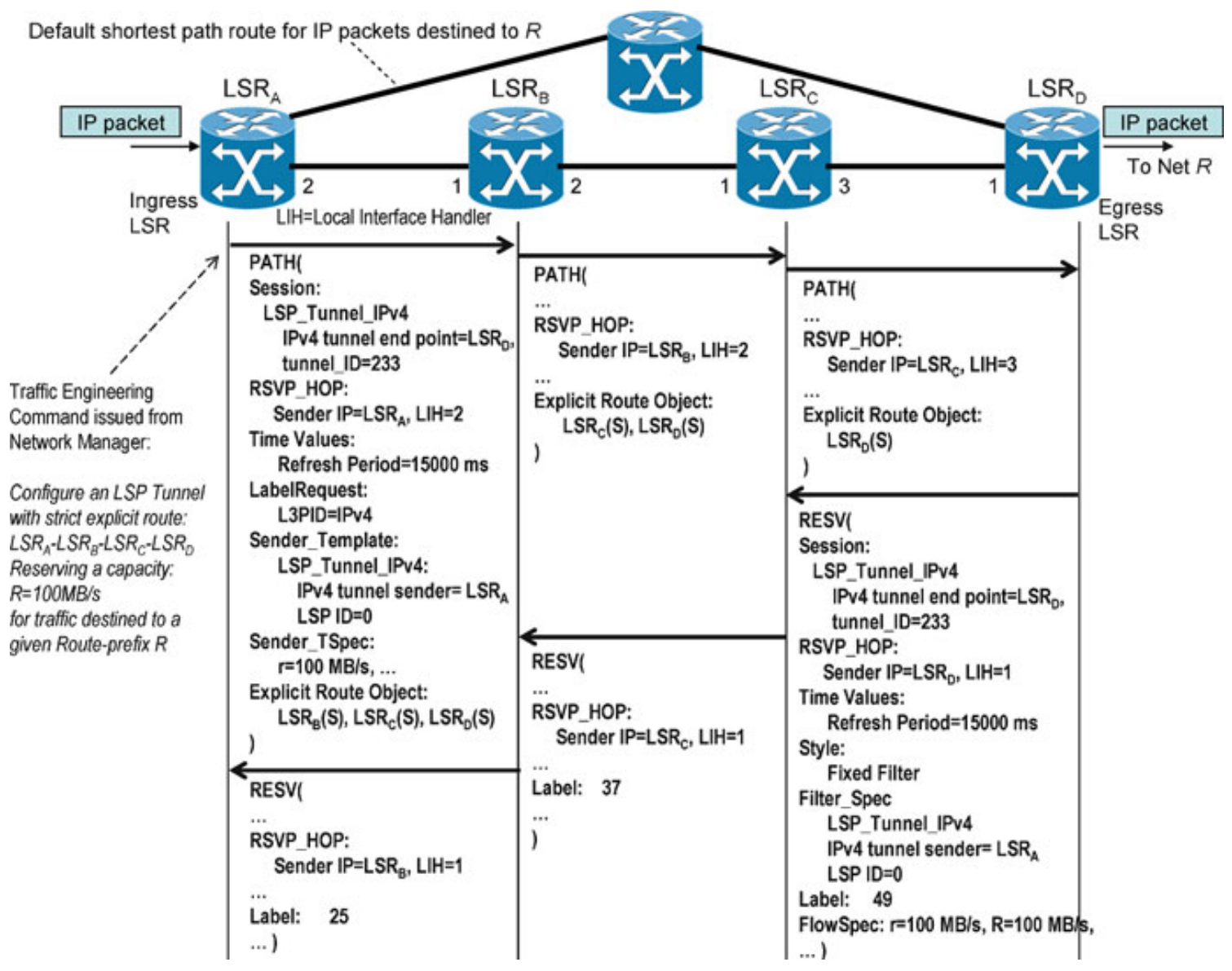

Fig. 2.2 MPLS traffic engineering: signalling an LSP tunnel with RSVP-TE

to set up traffic engineering LSPs, also known as MPLS LSP Tunnels ${ }^{1}$, and with GMPLS circuits, as described below.

We focus on this latter type of LSPs for its significance to optical networks. The idea is to be able to command an ingress or head-end LSR to set up an LSP to an egress or tail-end LSR that fulfils a set of constraints (e.g. have a residual capacity of $200 \mathrm{Mbit} / \mathrm{s}$ available). Given such command, the head-end router would compute the path and initiate the required RSVP-TE signalling (Fig. 2.2). For this to happen, the head-end router must have considerably extended link information about the network, such as maximum link bandwidth, maximum reservable bandwidth, currently unreserved bandwidth, the administrative group, etc. This is accomplished by means of TE extensions to an IGP protocol, such as OSPF-TE (RFC 3630 - traffic engineering extensions to OSPF).

\footnotetext{
${ }^{1}$ IP tunnels have also been used for simple traffic engineering operations. LSP tunnels have several advantages over IP tunnels, namely efficiency (label switching vs. route lookup forwarding), explicit route specification and forwarding (IP source routing could be used, but the processing of this IP option is less efficient than label swapping), immunity to routing instabilities and bandwidth reservation capability.
} 


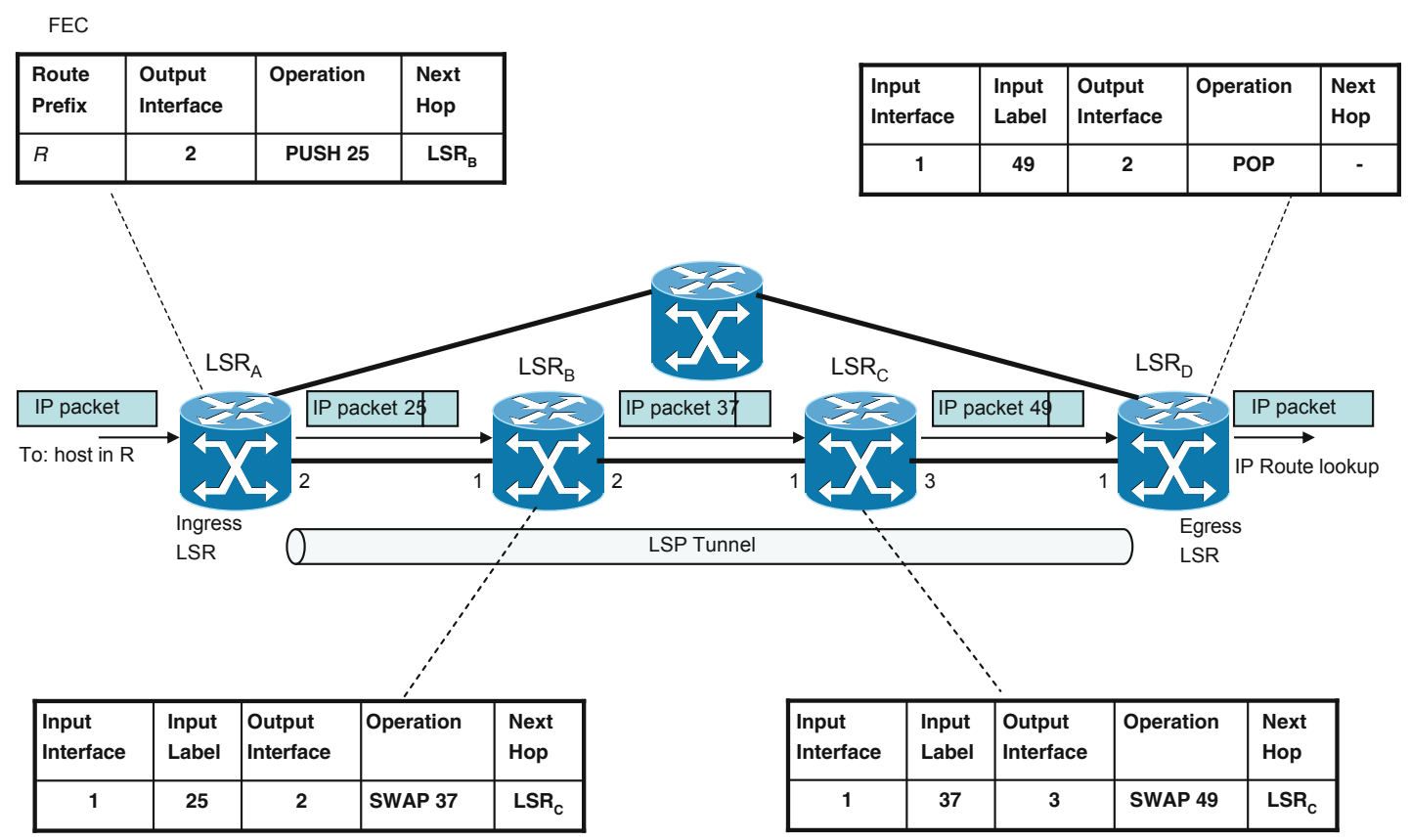

Fig. 2.3 Forwarding state after the LSP tunnel set-up

Once all the extended link information is known at the head-end and the LSP path is computed, the router starts the RSVP-TE signalling to set up the LSP Tunnel. RSVP-TE is an extension of the IETF resource reservation protocol RSVP, designed to deal with FEC-label assignments and reservations for traffic trunks (aggregation of flows of the same class to be placed in an LSP (see RFC2702)), rather than for individual IP flows. Figure 2.2 shows an example of LSP tunnel set-up and Fig. 2.3 depicts the resultant forwarding state along the new path for the diverted traffic. The additional route configuration required to reroute the traffic over the new LSP is not shown. Figure 2.3 also illustrates the forwarding of an IP packet over the LSP. Similarly to RSVP, RSVP-TE is a soft-state signalling protocol that implies periodic refreshing of signalled LSPs. RSVP-TE allows to specify the path from the source node in the ERO and, if a reservation is desired, a traffic descriptor, usually represented by a token bucket specification, denoting the average rate, peak rate and maximum burst size of the traffic trunk. In addition to improved traffic balancing across network resources, the LSPs created can also be used to divert part of the traffic from the shortest path, for example to deal with congestion, or as a predetermined backup route to fast reroute traffic traversing a failing network segment.

An interesting issue is path computation for the LSP Tunnel. This is out of the scope of RSVP-TE. In principle, within a single unstructured domain, OSPF-TE can supply the extended link-state information required for the head-end router to compute the shortest explicit route fulfilling the requirement (this is called constraint-based routing). However, this autonomous decision by the head-end LSRs can have the negative side effect on racing for network resources that induces 
potential blocking and degradation ${ }^{2}$ of LSPs requests. Therefore, the idea of having global sparsely distributed path computation elements (RFC4655) to calculate paths considering demands from all ingress routers in a correlated way is gaining interest. Especial mention deserves the ITU-T activity on MPLS. The multi-protocol properties of MPLS and its dual ability to build both IP-driven and IP-agnostic circuits are making MPLS one of the predominant technologies in today's packetswitched transport networks. This trend impelled ITU-T SG-15 to enhance its transport network model to embrace MPLS circuits and to try to provide service levels comparable to SDH with them. To prevent non-interoperable implementations of transport-specific MPLS architectures, IETF is working on a set of RFCs to support the requirements of ITU-T transport networks in terms of QoS, end-to-end OAM and protection. This version of MPLS, convergent with ITU-T TMPLS spec-ifications, is known as MPLS-TP (MPLS-transport profile) (Bocci et al. 2010). This transport profile is expected to become the basis for carrier-grade implementation of services, such as VPLS in the near future. Other alternatives under study by network operators, such as IEEE PBB-TE (provider backbone bridging-traffic engineering)(IEE8021), are only applicable to Ethernet-based backbones.

Before closing this short overview of MPLS concepts, we shall recall once more that the switching technology implied by MPLS is packet-based store-and-forward switching, not circuit-based cut-through switching. An attempt to integrate both worlds under the same framework came with GMPLS.

\subsection{Distributed Control of Optical Networks:GMPLS}

GMPLS (Mannie et al. 2004) is an extension of the MPLS Traffic Engineering scheme to support circuit switching networks, such as TDM and WDM Networks. In our context, GMPLS signalling is intended to drive the dynamic set-up of circuits in transparent or translucent optical networks by using the same signalling protocols as MPLS-TE and extensions.

The technological shift to dynamically switched circuits has still a long way to go before multivendor interoperability of optical equipment is in place. The reason for this is the inherent complexity of analogue optical interfaces in WDM, hindering consensus between vendors. Therefore, nowadays, circuit set-up and management in optical networks is single-vendor and mainly performed manually by operators from a central network operation centre. Provisioning is facilitated by proprietary applications that connect to every node to configure cross-connections and carry out other OAM tasks. It is common to find vendors offering web-based interfaces for circuit provisioning and management of their equipment. GMPLS tries to change

\footnotetext{
${ }^{2}$ This is especially the case of GMPLS in DWDM, as explained later in the chapter, where distributed and concurrent requests add extra burden to the already complex process of optical circuit provisioning.
} 
this centralised approach to a more distributed one (Banerjee et al. 2001). In GMPLS, all nodes exchange control information over a signalling channel, and connection set-up can be initiated by the head-end of a connection (following a TE request from the NMS) and is realised by the exchange of messages between adjacent nodes using RSVP-TE. This requires a control network based on IP, i.e. optical switch's control electronics need to have a TCP/IP stack, an IP address, run RSVP-TE and OSPF-TE or ISIS-TE, alongside with a LMP. Thus, a single control protocol could eventually provide end-to-end circuit provisioning across routers, SDH multiplexers and optical switches, as well as a unified framework for protection, restoration, monitoring, management, etc., including all layers and switching devices involved in a connection.

In the beginning, GMPLS was regarded as a competing alternative to ITUT ASON signaling (G.8080/Y.1304), but the open nature of these signalling specifications (G.7713 (G7713)) made it possible to include IETF GMPLS signalling protocols with specific extensions (RFC4974 (RFC4974)) as an ITU-Trecommended alternative to ITU-T PNNI/Q.2931 (G.7713.1/Y.1704.1 (G77131) and G.7713.2/Y.1704.2 (G77132), respectively). Furthermore, the OIF has tried to adopt the best of ASON and GMPLS concepts to define and impulse the take-up of specific signalling protocols. These recommendations include both signalling protocols too and include guidelines for interworking of PNNI and RSVP-TE/ OSPF-TE subnetworks.

Next, we briefly outline the main changes introduced by GMPLS with respect to MPLS. A recommended, more detailed overview can be found in Halabi (2003).

- Circuit switching network support. Compared to MPLS virtual circuits over packet-switched networks, GMPLS makes true circuits over a circuit-switched network; these circuits are still called LSPs. In GMPLS, the data units do not carry an explicit label; the label is only managed by the control plane to identify the timeslot, wavelength or port through which the connection data must be switched. To deal with each specific technology, GMPLS defines five types of LSR interfaces: PSC, L2SC (e.g. ATM), TDM (e.g. over SONET/SDH channels; in this case, forwarding is based on the incoming timeslot of data, i.e. the implicit label is the timeslot), LSC (e.g. optical cross connect with wavelength switching granularity; the implicit label is the lambda) and FSC (Fibre-switch capable, e.g. photonic cross-connect with light switching granularity; the label is the port number). The interface switching capability descriptors are advertised by means of the routing protocol.

- Link management. Unlike MPLS LSRs, GMPLS nodes may have many links with the neighbouring nodes. In order to drive bundles of WDM channels in a suitable and efficient way, instead of overloading the IGP with new functions, a specific LMP (RFC4204) has been proposed to manage TE links between every two neighbouring nodes over a common control channel. Link provisioning, bundling, protection, fault isolation, signalling control channel monitoring, connectivity verification, configuration and verification are some of the functions carried out by this protocol. 
- Routing: topology and resource discovery. In GMPLS, network nodes run IP routing protocols with Traffic Engineering extensions, usually OSPF-TE, to convey not just topology information but also available link capacity, termination capabilities and protection properties. To deal with multiple links between adjacent nodes in an efficient way, the routing allows to bundle these links into a TE link and to announce them as such, summarising the information of its component links. Component links can be uniquely identified by tuples $<$ node ID, link bundle, link identifier $>$. This is called "unnumbered link" support because no explicit subnet is built per individual link or link bundle. Other information conveyed by routing protocols for GMPLS is the SRLG and the link protection type. A set of links may constitute a "shared risk link group" (RFC4202) (RFC4202) if they share a resource (e.g. a fibre conduit) whose failure may affect all links in the set. The SRLG information is a list made up with all the SRLGs that the link belongs to. An SRLG is identified by a 32-bit number that is unique within an IGP domain. The SRLG of a LSP is the union of the SRLGs of the links in the LSP. Disjoint SRLG LSP path computation is important to build protection circuits. The link protection type information is used by TE algorithms to search for paths with a target protection level. The protection types defined are extra traffic, unprotected, shared, dedicated 1:1, dedicated $1+1$ and enhanced.

- Signalling. In order to support the new interface classes, a number of changes on signalling are required. The most relevant follow:

- Generalised labels are introduced to identify time slots, wavelengths range in a waveband being switched, fibres in a bundle and the MPLS label. Generalised Label requests determine the technology-specific LSP type being requested. Unlike MPLS, GMPLS permits an upstream node to suggest a label to the downstream node before the label mapping is received from downstream, and it is possible to constrain the label range to be used to set up LSPs between peers. This is especially useful to deal with wavelength switching and conversion limitations of optical cross-connects. Furthermore, it is possible to determine a common end-to-end label for all-optical LSPs without wavelength conversion.

- Bidirectional LSPs. Unlike MPLS-TE, GMPLS enables the specification of both unidirectional and bidirectional LSPs to improve set-up latency and path consistency, especially for networks with bidirectional circuits (e.g. $\mathrm{SDH} / \mathrm{SONET}$ ).

- Control/data plane separation. In MPLS-TE, signalling normally uses the same interfaces used for data, and hence data and control links share their fate. In GMPLS, control and data interfaces are usually different. This implies the need for mechanisms for proper identification of the control and data channels being controlled, and a differentiated fault handling of data, control channel and nodes. Consequently, the application of the soft-state model used in MPLS RSVP-TE is diluted in GMPLS since control plane and data plane failures may not be correlated, and hence LSPs are not released whenever a 


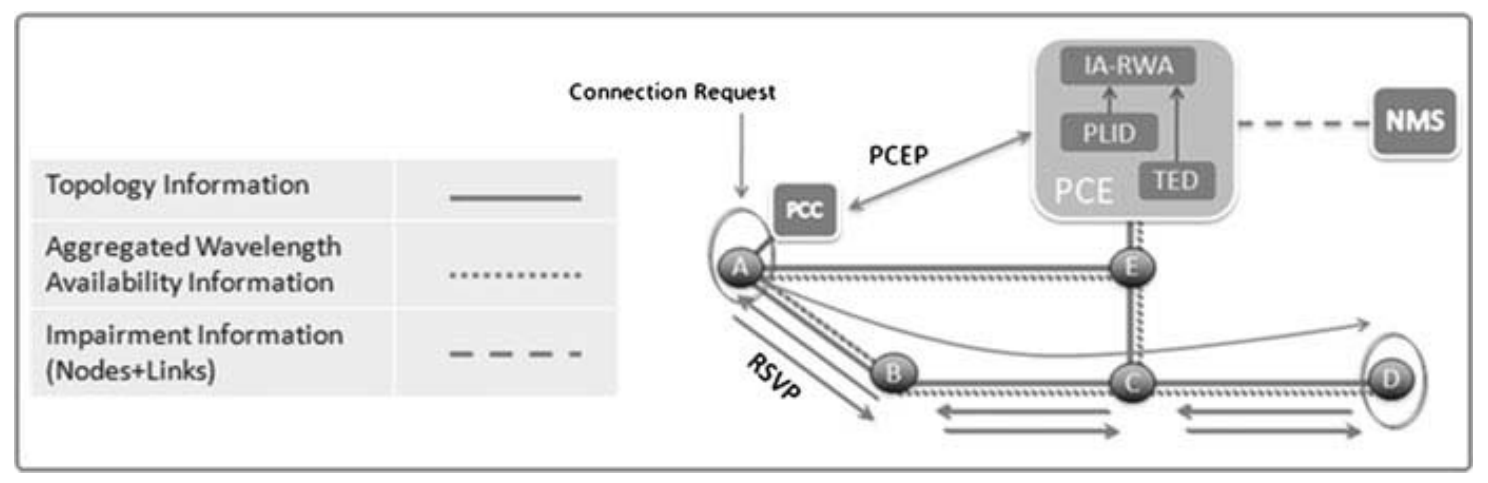

Fig. 2.4 Centralised approach - topology and aggregated wavelength availability information are collected through OSPF-TE, and impairment information through the NMS

sequence of path/resv messages are missing. Control plane restart processes (RFC3473, RFC5063) allow adjacent nodes to resynchronise their control plane state to reinstate information about LSPs that have persisted in the data plane after a node or link failure. GMPLS also proposes a new RSVP-TE Notify message to notify errors to non-adjacent nodes along routes that are not tied to the connection path.

- Hierarchical LSPs. Signalling must support LSP nesting even with unnumbered links in order to improve the scalability of GMPLS (Kompella 2005). An LSP may be advertised as a TE link for use within the same instance of the control plane as was used to set up the LSP.

In transparent optical networks, it is important to assess the signal quality of a path that needs to be established from a source to a destination node. Due to the lack of electrical regeneration, optical signal impairments accumulate along the path. Consequently, it is not sufficient anymore to just find free resources in the network to establish a feasible path. However, there is not yet a standard to support physical impairments in the GMPLS framework. In order to cater for this problem, there are a number of solutions that have emerged over the last couple of years. There are two fundamental ways to incorporate physical impairments in GMPLSbased optical networks: a centralised approach and a distributed approach. They are outlined and discussed next.

1. Centralised approach

In the centralised approach (Fig. 2.4), an entity referred to as the PCE (RFC4655) is employed in the network and is responsible for computing the routes for the connection requests within its domain. The PCE maintains a centralised TED (e.g. populated using an OSPF-TE routing protocol) for the network topology and aggregated wavelength availability information for all the links in the network. In addition, the PCE maintains a PLID, e.g. populated by the NMS. PLID can either contain information related only to static impairments or include also dynamic impairments information, which is updated at regular intervals. Alternatively, the PCE may fetch this information directly from the ingress nodes. 
There are different ways to employ a PCE in this centralised approach. One possibility is to have all connection requests sent directly to the PCE from the ingress nodes, also known as path computation clients in the PCE terminology, for the path computation. The PCE, in turn, responds to the path computation clients with the computed paths. A signalling protocol (e.g. RSVP-TE) is then used to establish the path (Castoldi et al. 2007). Another possibility is to have the NMS responsible for coordinating with the PCE for the path computation and for establishing the computed paths, manually or through a signalling protocol (Tsuritani 2006).

On the positive side, this approach does not require any modification to either the signalling or the routing protocol, while impairments can be catered for, with minimal effort, during the path computation process in the GMPLS framework. Furthermore, since both the TED and PLID are maintained at the PCE, an IA RWA algorithm (see Chap. 2.4) considering the TE information together with the physical impairments can be employed at the PCE to compute an optimal path from source to destination.

However, there are a number of issues associated with this approach. First, this solution does not scale well, particularly when there is only one PCE available per domain. This is mainly because of the intensive computation associated with constraint-based routing, in general, and with IA-RWA, in particular. Moreover, a single PCE approach is vulnerable to TED and PLID errors and failures, aside from the inevitably delayed response to dynamic changes (e.g. link failures) in the data plane, which likely demand recalculation of many paths at once. Finally, there is a certain control overhead associated with the PCEP for every exchange of PCEP related messages. However, this overhead can be mitigated by enabling the bundling of connection requests (Ahmed et al. 2010), which provides also an opportunity for concurrent path optimisation, an efficient way of reducing the connection blocking probability.

2. Distributed approaches

Distributed solutions can be further categorised in two groups: routing-based approaches and signalling-based approaches, which are outlined and discussed next.

- Routing-based approach

In the routing-based approach (Strand et al. 2001), OSPF-TE is extended to convey impairment parameters between nodes in the network. For this purpose, some new top-level type, length and value (TLV) objects are incorporated. In this approach, the impairment parameters for the network links are disseminated via OSPF-TE in the same way as network topology, QoS and TE information. At each routing node in the network, a global PLID database is maintained in addition to the TED (Martinez et al. 2006), with the up-to-date physical-impairment-related information for all links in the network. For each incoming connection request, the ingress node runs an impairment-aware routing algorithm (Chap. 2.4.2 presents some candidates) to determine a feasible path from source to destination (see Fig. 2.5). Next, 


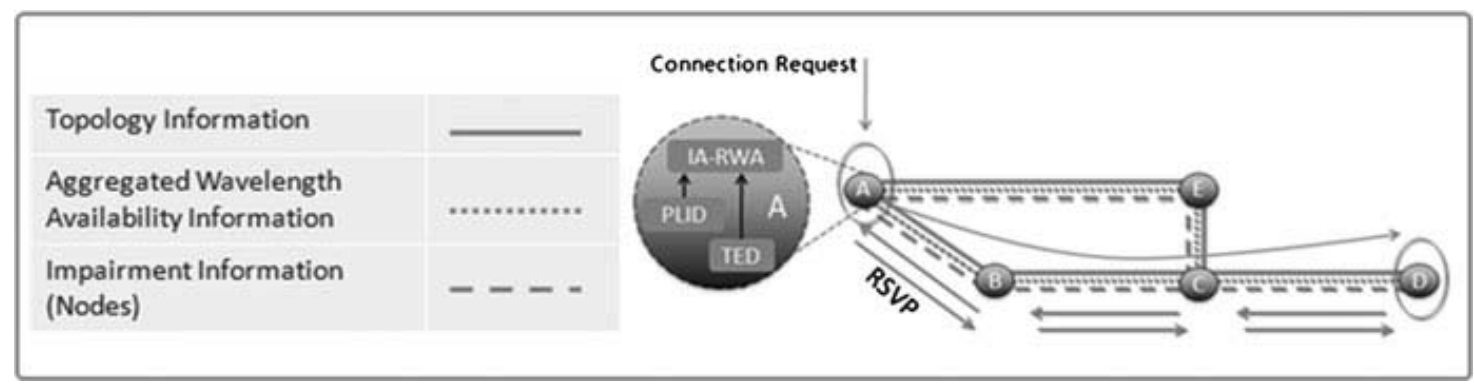

Fig. 2.5 Distributed routing approach - topology aggregated wavelength availability information and impairments information are all collected through OSPF-TE

similarly to the centralised approach, RSVP-TE-based signalling is used to establish the path. Normally, a set of K-shortest paths is computed using the IA-RWA algorithm in order to provide alternative routes in case the path establishment on the first computed path fails during the signalling phase.

This approach has the advantage that a global TED and PLID are maintained locally at all the nodes with path computation capabilities. In addition, path set-up time is shorter, assuming that there are no significant convergence time issues for OSPF-TE. However, there are also several problems associated with this approach. First of all, it does not scale well. In particular, the control overhead can grow very fast if the network domain is large and if there are frequent changes in the TE- and PLID-related information. An approach to mitigate the control overhead has been presented in (Halabi 2003), where the authors propose a TI-LSA. According to the TI-LSA idea, the OSPF-TE LSA flooding principle is modified to take advantage of the node's TI. TI-LSA limits the number of flooded entries by constraining the scope of advertisements to only the nodes that may require that information, i.e. within the boundaries of the node's TI. As a result, the TI-LSA protocol may cope with increasingly large size networks and represents a scalable solution to the problem of topology discovery and update when the TI size is small relative to the whole network. A second problem with this centralised approach is related to inconsistencies in the TED and PLID, mainly due to information propagation latencies. These inconsistencies may lead to suboptimal routing and may ultimately increase the average LSP request blocking probability and/or the resource consumption. The applicability of this approach also depends upon the processing power of each NE. If there are NEs with low computation power, the processing of the LSA may take longer, hence an increased risk of database inconsistency among different nodes. This, in turn, has a direct effect on the LSP request blocking probability, as demonstrated by the work in (Salvadori et al. 2007a). It can be concluded that this approach is not appropriate for large and highly dynamic network scenarios. Another drawback is the significant modifications that need to be incorporated in OSPF-TE. 


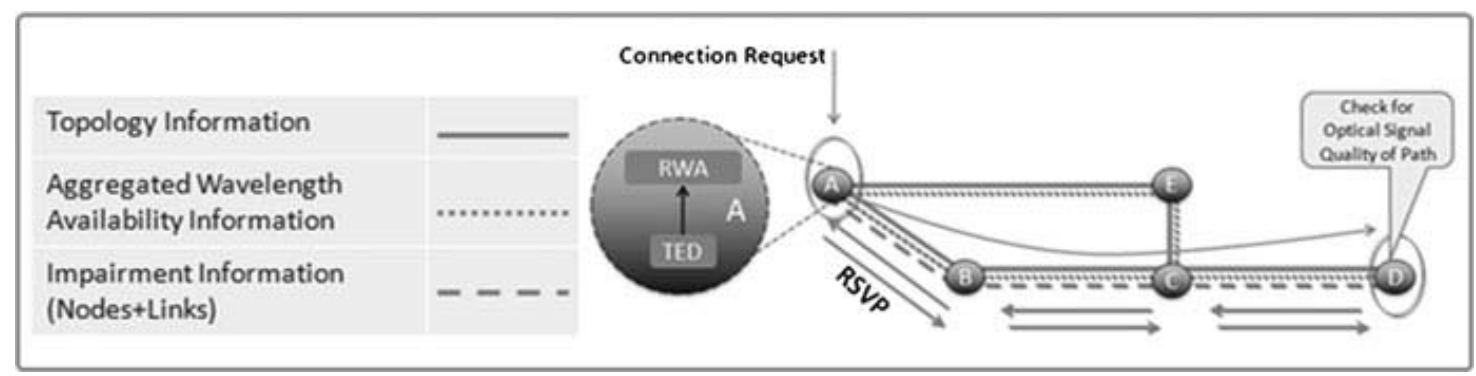

Fig. 2.6 Distributed signalling approach - topology and aggregated wavelength availability information collected through OSPF-TE, and physical impairments information collected during signalling process

In (Salvadori et al. 2007a), a different solution for the distributed routing approach is specified. Here, detailed impairment-related models are uploaded to all the nodes in the network, while only consolidated wavelength availabil-ity information, provided by OSPF-TE, is utilised by the nodes to estimate the effect of static link impairments. However, it is assumed that all NEs are of the same kind. In fact, when different optical devices (e.g. from different vendors) are incorporated in a network domain, the estimation of end-to-end LSP impairments becomes much more difficult, given that different models need to be considered hop-by-hop.

- Signalling-based approach

A lot of recent work addresses a distributed solution referred to as signallingbased approach (Cugini et al. 2004). As opposed to routing-based solutions where OSPF-TE was extended to distribute physical layer information, in a signalling-based approach, RSVP-TE is extended with more objects to incorporate and communicate impairment-related information. Similarly to the routing-based approach, a local PLID is maintained at each node, but this time, only impairment information for the current node and its associated links is stored. In a signalling-based solution, the RSVP-TE Path message collects the impairment-related information along all the traversed links and nodes from the source to destination. At the destination node, an evaluation is then made, based on the collected impairment information, to get an estimate of the optical signal quality and to decide whether a path should be accepted (i.e. an RSVP-TE Resv message is sent on the reverse path from destination to source) or it should be rejected because of unsatisfactory signal quality (i.e. a RSVP-TE PathErr message is sent back to the ingress node). This is illustrated in Fig. 2.6 and compares to the IA-RWA approach outlined in Sect. 2.4.2.6. Note that in this case the ingress node computes a set of K-shortest paths (possibly link-disjoint) using an RWA algorithm based only on resource availability information and tries to establish a path using the modified RSVPTE protocol. If the first path cannot satisfy the signal quality requirement, then the next one is tried and so on. The decision on whether to use a computed path or not is made at the destination (egress) node. Furthermore, in (Sambo et al. 
2006), a modification for the PathErr message is proposed. If the egress node fails to establish the path, it may also send back some additional information about the reason of the failure (e.g. insufficient signal quality on a link along the route). In this way, ingress node can get an estimate of the signal quality of a number of links and nodes in the network. It can store this information in the local PLID, populate it and use it for the new connection requests. This kind of the signalling approach is referred to as lightpath provisioning using signalling feedback.

A possible drawback of the signalling-based approach is that it uses RWA based only on the resource availability information, and path feasibility in terms of optical signal quality is only checked at the egress node. Therefore, the computed path may not be optimal in terms of signal quality. Due to this reason, it may increase the average path set-up delay because it may take several attempts before a path is finally established, e.g. a route with an acceptable signal quality is found. A possible solution to this problem is presented in Salvadori et al. (2007b) where, rather than trying to establish the path using a set of computed K-routes in a sequential manner, a Path message is sent along all the computed K-routes in parallel to try to establish a path with an acceptable signal quality, although in this case, there will be a higher signalling control overhead for each connection request. Another variant of this approach is also proposed in (Salvadori et al. 2007b) where the route is computed on a hop-by-hop basis so that each node only considers information related to its directly connected links.

On the positive side, this approach requires minimal changes to RSVP-TE and no changes at all to OSPF-TE (Cugini et al. 2005). Furthermore, the computational load exerted on the ingress nodes is much less intensive (i.e. absence of IA-RWA). Also, the performance of this approach is less prone to the dynamism in the network since local PLID is not populated through the OSPF-TE and hence not dependent on the significant LSA processing delays in larger and more dynamic network scenarios. For the above-mentioned reasons, this approach is much more scalable. Finally, note that this approach can also cater for the intra-node impairment parameters in addition to the link-based impairments as in the routing-based approach.

\subsection{Convergence Between Metro and Access}

The development of transmission techniques is increasing the maximum distance and the number of users served by the access networks. On the one hand, the provided data rate is significantly increasing, from $155 \mathrm{Mbit} / \mathrm{s}$ to currently under deployment $1 \mathrm{Gbit} / \mathrm{s}$ and $2.5 \mathrm{Gbit} / \mathrm{s}$ of IEEE EPON and ITU-T GPON, while also the next recommendations are already focusing in $10 \mathrm{Gbit} / \mathrm{s}$ as the $10 \mathrm{Gbit} / \mathrm{s}$ Ethernet Passive Optical Network standard (10 G-EPON) recently approved in September 

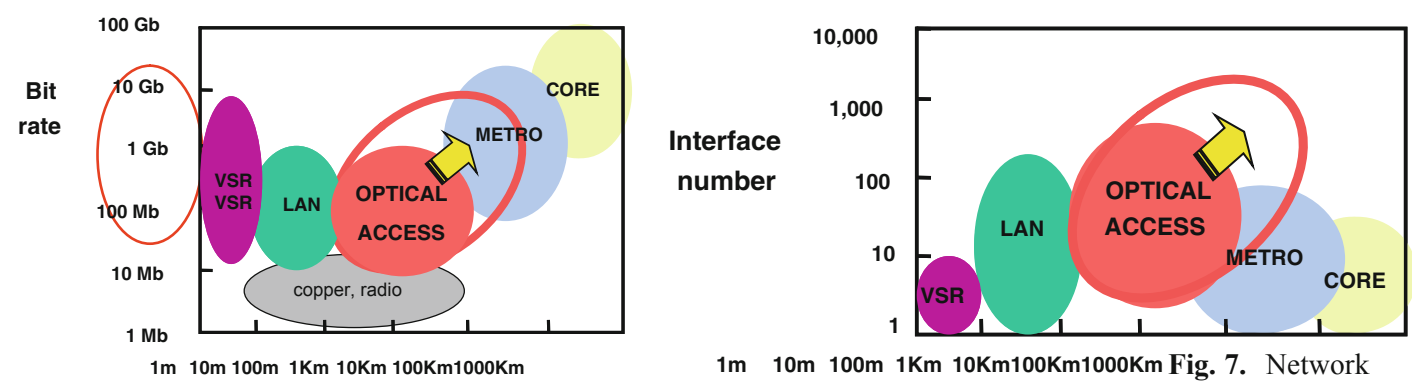

Fig. 2.7 Network segment definition in terms of bit rate, interface number and distance served

2009 as IEEE 802.3av (IEEE8023). On the other hand, ITU-T and FSAN are also working on the standardisation of the XG-PON (gigabit passive optical network). In parallel to the progress in standardisation of higher bit rates, also the maximum reach distance of access networks and the maximum number of users have been significantly enhanced, and new recommendation proposals are including signal regeneration techniques as the "extender boxes" (EB) over PONs (IEEE8023) which can offer significant extra optical budget (up to $30 \mathrm{~dB}$ ). The EB is inserted between the OLT and ONUs to provide extra budget to the system while being transparent to protocols and ranging process. In February 2008, the ITU GPON standard (Huang et al. 2005) for optical reach extension, also known as G984.re, was finalised. This standard defines $60 \mathrm{~km}$ reach with an optical budget higher than $27.5 \mathrm{~dB}$ achieved in both spans adjacent to the EB. Moreover, compatibility with existing equipment is maintained. Both optoelectronic regeneration and optical amplification are considered as solutions for the EB (ITUG984).

The evolution of the demand in date rata and power budget in access networks, leading to extended reach and higher number of users, is forcing the convergence between access and metro transport networks, as shown in Fig. 2.7.

Several research projects are focused on the convergence process as ISTMUSE, Photonic Integrated Extended Metro and Access Network ISTPIEMAN, FibreOptic Networks for Distributed Extendible Heterogeneous Radio Architectures and Service Provisioning ISTFUTON and Scalable Advanced Ring-based Passive Dense Access Network Architecture ISTSARDANA. While the projects, MUSE, PIEMAN and FUTON, are considering the use of elements requiring electrical supply in the outside fibre plant of PON, the SARDANA project focuses on maintaining a fully passive outside plant. Obviously, some kind of optical regeneration is required for providing the extra budget of converged transport and access networks. This is achieved in SARDANA by means of remotely pumped optical amplification as Raman and/or hybrid Raman and EDF. We describe the main characteristic of SARDANA approach and the physical monitoring and compensation challenges for CD, Rayleigh Back-scattering RBS, OSNR and physical link protection.

The SARDANA network implements an alternative architecture of the conventional tree WDM/TDM-PON. It consists in the organisation of the optical distribution network as a WDM bidirectional ring and TDM access trees, interconnected by means of scalable optical passive add \& drop RN, as shown in Fig. 2.8. 


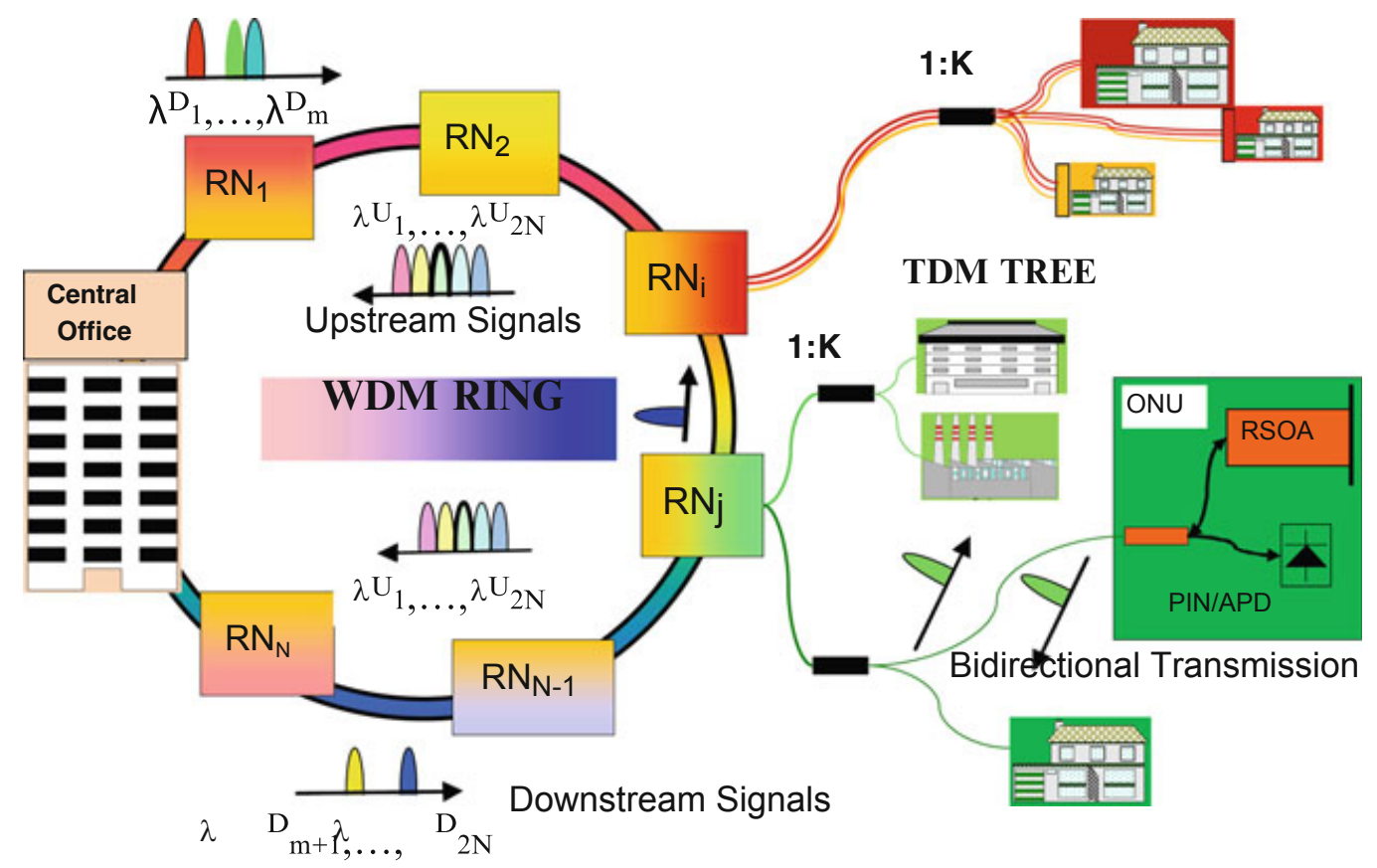

Fig. 2.8 Network architecture and implemented test bed comprising a remote node and reflective SOA as colourless ONU

The proposed network aims serving more than 1,000 users spread along distances up to $100 \mathrm{~km}$, at $10 \mathrm{Gbit} / \mathrm{s}$, with $100 \mathrm{Mbit} / \mathrm{s}$ to $10 \mathrm{Gbit} / \mathrm{s}$ per user in a flexible way (Bock et al. 2007; Lazaro et al. 2007a; Lazaro et al. 2008).

The ring and tree topology can be considered as a natural evolution from the conventional situation where Metro and Access networks are connected by heterogeneous $\mathrm{O} / \mathrm{E} / \mathrm{O}$ equipment at the interfaces between the FTTH OLTs and the Metro network nodes towards an integrated Metro-Access network. In this case, the network is covering similar geographical area, users and services, but the equipment, previously scattered, will now sit at a unique $\mathrm{CO}$, and implementing an all-optical passive alternative, operating as a resilient TDM over WDM overlay. Depending on the scenario, the ring and tree mixed topology optimises the usage of the fibre infrastructure in the ODN and also offers enhanced scalability and flexible distribution, as new RNs can be installed.

This network transparently merges TDM single-fibre tree sections with a main WDM double-fibre-ring by means of the passive RNs. The $100 \mathrm{~km}$ WDM ring transports, e.g. 32 wavelengths for $>1,000$ users if the TDM trees implement a splitting factor of $1: \mathrm{K}=32$, and only 1 wavelength per TDM tree is required. Protection and traffic balancing properties of the network are provided by the ring configuration and the design of the RNs (Prat et al. 2009), providing always a connection between each $\mathrm{RN}$ and the $\mathrm{CO}$ even in the case of fibre cut.

Physical monitoring is proposed by using several techniques. Implementation of OTDR techniques can be used by adapting these techniques to a WDM network, such as the SARDANA network (Teixeira et al. 2009). 


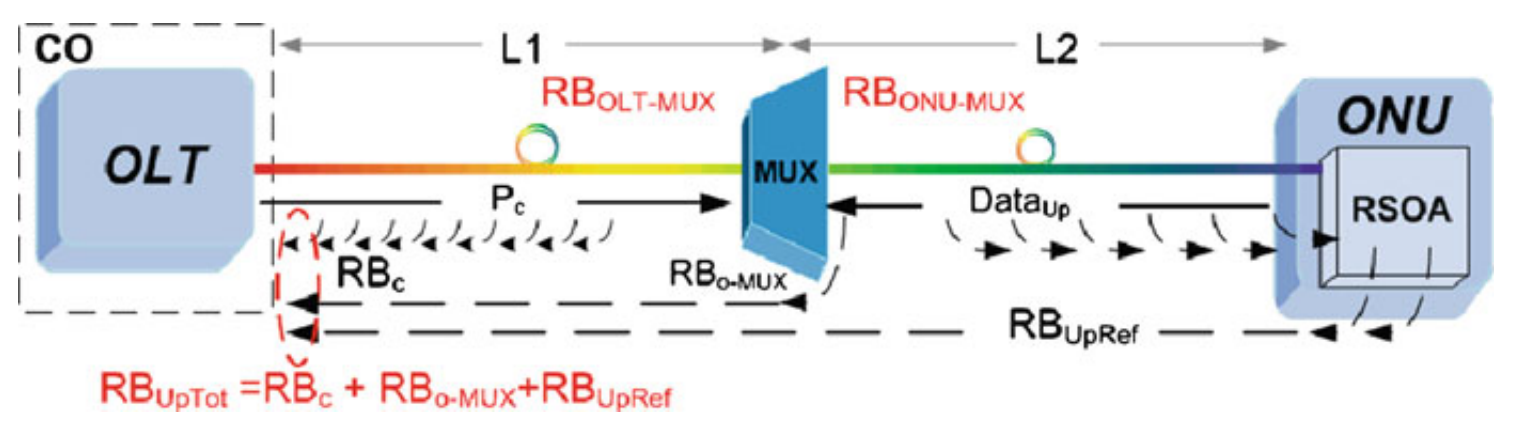

Fig. 2.9 Schematic of a plain WDM-PON with MUX at the fibre plant

In addition to the problem of physical layer monitoring, several transmission challenges are faced as the network evolves towards a more transparent, passive and extended network. Some of the transmission impairments are not specific from SARDANA network but are common for extended WDM PONs.

For example, Rayleigh Backscattering is a common challenge for single-fibre networks with centralised optical signal distribution. As shown in Fig. 2.9, the $\mathrm{C} W$ carrier signal provided by the $\mathrm{CO}$ is modulated with the user data at the ONU and back reflected in the upstream direction on the same wavelength. In this full-duplex single-fibre bidirectional transmission context, RBS is a dominant impairment. For compensating this impairment, several approaches can be followed: frequency dithering of the optical source at the CO (Lazaro et al. 2007b); wavelength shifting or the centralised distributed signal by the ONU (Omella et al. 2009a); the analysis the best network design and ONU gain for reducing its impact (Lopez et al. 2010). Following this approach, it is possible to analyse the most adequate location of the MUX in the PONs and to determine the optimum ONU gain on each case.

Figure 2.10 shows the simulated and experimental results of the BER values obtained as a function of the MUX position and the different ONU gain values.

Regarding the extension of the access networks, the incorporation of the transparent interfaces between the transport and the access networks makes the accumulation of OSNR as one of the more challenging impairments. As in the case of RBS, this is not a challenge which is unique to SARDANA network but also for other transport plus access converged networks.

Also, the WDM PONs can be considered a transport plus access network, as a trunk fibre is used for transporting downstream and upstream signals through longer distances, as shown in Fig. 2.11.

In this case, a ROPA is analysed as box extender for increasing the transport (trunk section) power budget while maintaining the access budget. Figure 2.12a shows as a reference, the typical access budget (G.984.2, 2.4/1.2 Gbit/s) for range from 13 to $31 \mathrm{~dB}$ at $0 \mathrm{~dB}$ of trunk budget, providing $3 \mathrm{~dB}$ margin to the $28 \mathrm{~dB}$ of maximum access losses specified by $\mathrm{B}+$. The small trunk budget margin (shown by the vertical line) from 0 to $4 \mathrm{~dB}$ is compatible with the $15 \mathrm{~dB}$ ODN loss variations and maximum loss of $\mathrm{B}+$ recommendation (marked by the box). Using the same RX and TX and including a ROPA, Fig. $2.12 \mathrm{~b}$ shows that the ROPA can provide 


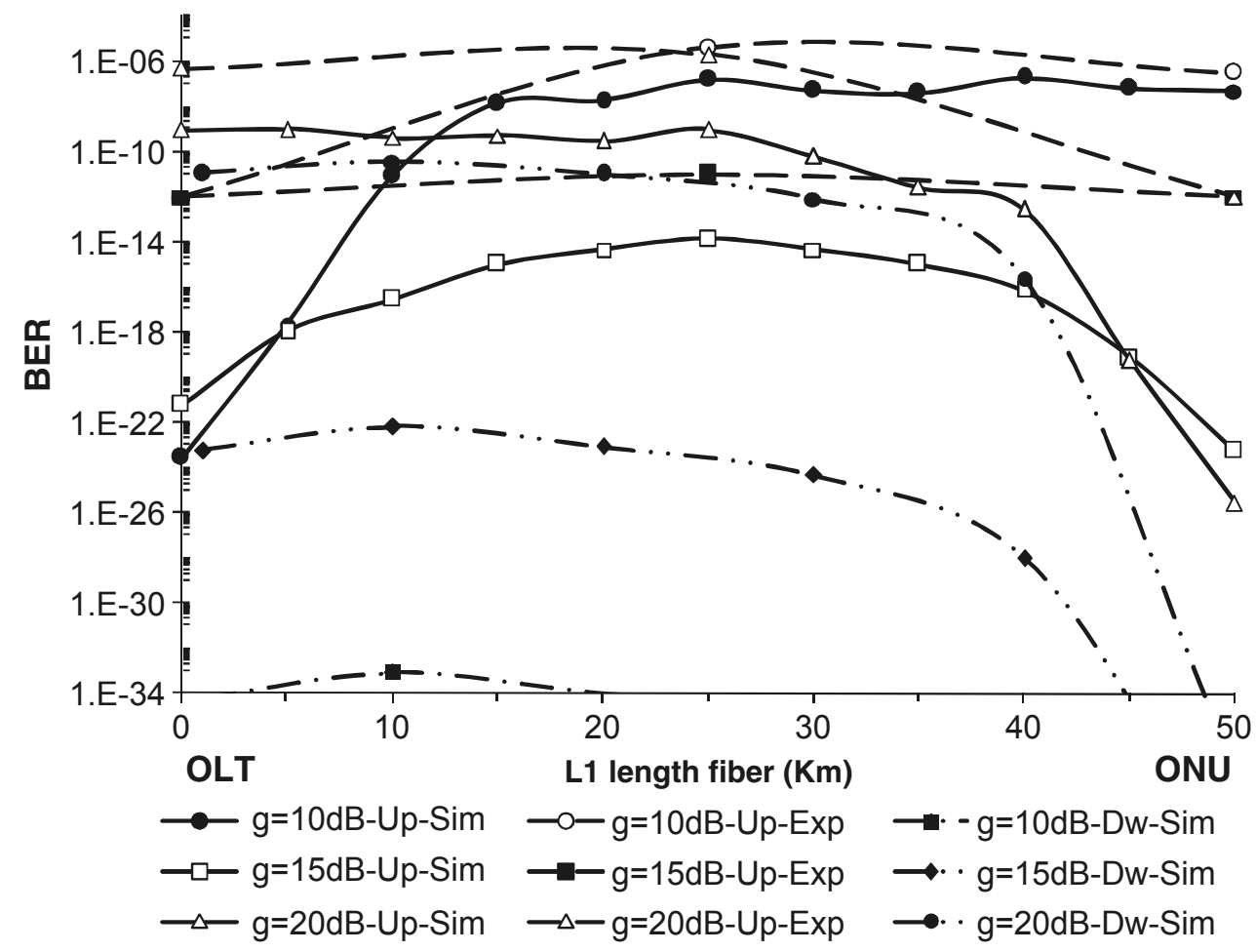

Fig. 2.10 BER as a function of the MUX position to different ONU gain values (simulations and experimental results)

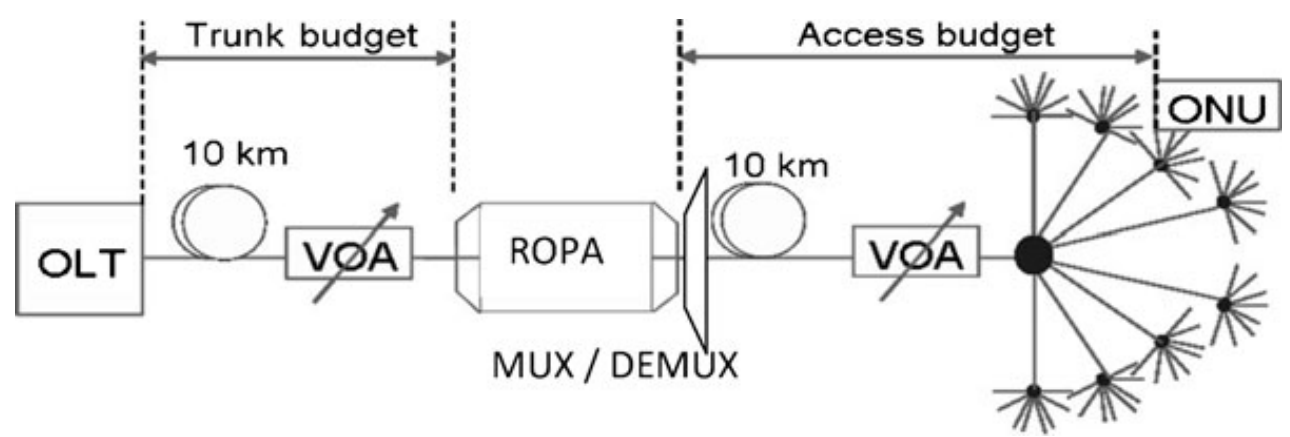

Fig. 2.11 Architecture analysed for the ROPA as extender box, including a Mux/Demux for the overlaying of several G-PON architectures in a WDM/TDM network

enough gain to provide an extra trunk budget from 0 to $14 \mathrm{~dB}$ compatible with $\mathrm{B}+$ access budget from 13 to $28 \mathrm{~dB}$ by implementing a ROPA with $15 \mathrm{~m}$ of EDF and providing $20 \mathrm{dBm}$ of pump, at $1,480 \mathrm{~nm}$, at the ROPA. Thus, a total budget of $42 \mathrm{~dB}$ is achieved.

In SARDANA architecture, the power budget extension is performed by hybrid Raman and EDF amplification. Remote pump power is transmitted through both upstream and, if required, downstream fibres.

A similar analysis to the WDM-PON can be done, while in this case, due to the higher complexity of the network, the BER values for the furthest ONU in resilience mode (a fibre failure has been produced and all the traffic has to be directed through one side of the WDM ring architecture) are shown in Fig. 2.13. It is shown that the 

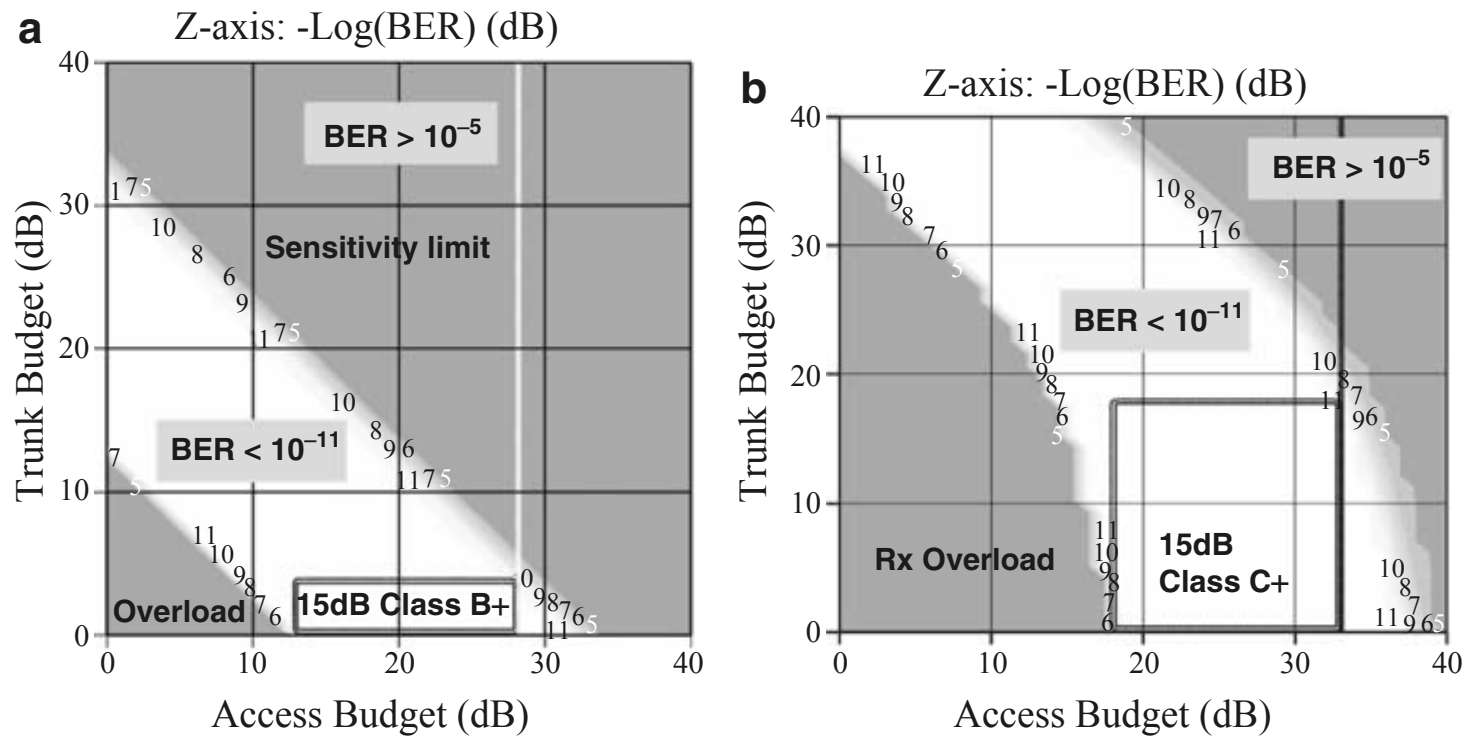

Fig. 2.12 (a) BER values for a G-PON (2.4/1.2 Gbit/s) using PIN detectors with minimum sensitivity and overload values $-28 \mathrm{dBm}$ and $-8 \mathrm{dBm}$, respectively, and TXs providing the maximum mean launched power $(+1.5 \mathrm{dBm})$; (b) BER values with a ROPA for 8 WDM wavelength channels for a ROPA with $15 \mathrm{~m}$ of HE980 EDF and $20 \mathrm{dBm}$ of pump power, the vertical line indicating the maximum access budget for $\mathrm{B}+(28 \mathrm{~dB})$; both for downstream transmission at the central channel at 1,550 nm (Max BER: 10-11 and $\min$ BER: 10-5)
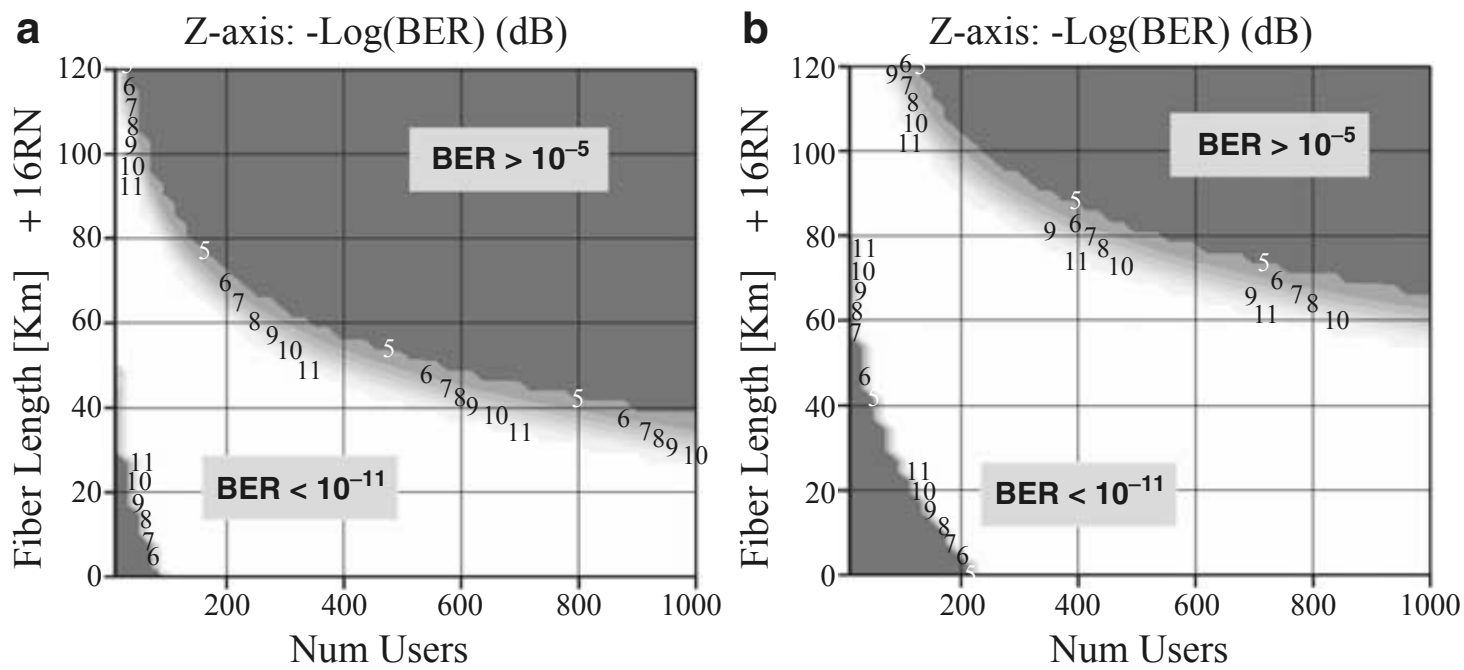

Fig. 2.13 (a) DS BER values for the furthest ONU in resilience mode of a Raman + In-line remotely pumped SARDANA network of 32 channels sending $3 \mathrm{dBm}$ per channel from the OLT, $50 \%$ splitting signal at the ONU, with $1.2 \mathrm{~W}$ of pump at $1,480 \mathrm{~nm}$ for both US/DS fibres and an ONU RX showing a sensitivity of $-24 \mathrm{dBm}$ at $10 \mathrm{Gbit} / \mathrm{s}$.; (b) DS BER values $2.4 \mathrm{~W}$ of pump at $1,480 \mathrm{~nm}$ both US/DS fibres

combination of Raman and in-line EDF amplification can provide adequate signal quality for up to $100 \mathrm{~km}$ reach and 128 users (under the assumption of first 20-25 km of the ring free of RNs) or $20 \mathrm{~km}$ reach and 1,024 users by remotely pumping with 
$1.2 \mathrm{~W}$ of pump at 1,480 $\mathrm{nm}$ for both US/DS fibres and a higher number of users and distances, by rising the available pump power per fibre to $2.4 \mathrm{~W}$, as shown in Fig. 2.13b.

The SARDANA project focuses on providing services up to $10 \mathrm{Gbit} / \mathrm{s}$. The transmission of signals at this data rates through distances in the range of $100 \mathrm{~km}$ results in the $\mathrm{CD}$ impairment. In this project, two approaches are analysed: compensation of fibre $\mathrm{CD}$ by dispersion-compensating fibres located at the $\mathrm{CO}$ and by electronic equalisation techniques (Omella et al. 2009b).

\section{Monitoring and Signal Processing in Optical Networks}

\subsection{Monitoring}

The engineering of high-bit-rate WDM optical transmission systems requires a careful control of each channel characteristic in order to limit the detrimental effects of the different types of physical impairments taking place in single-mode optical fibres. The initial values of the parameters, set at the system installation, may need further adjustment due to many reasons:

- Evolution of the characteristics of optoelectronic devices

- Fluctuation of the fibre characteristics

- Deployment of additional wavelength channels

- Upgrade of the line rate of the channel

The required flexibility tends to be increasingly important because optical transport networks become more dynamic and transparent. For instance, as ROADMs are now implemented in long haul transmission systems and metropolitan rings, the different wavelength channels may experiment a new transmission path according to the actual ROADM configuration. This issue will become even more complex in the case of meshed networks using transparent (i.e. without optoelectronic regeneration) or partly transparent networks based on optical cross-connects.

Considering all these possible changes in the network, it seems quite impossible to base the control of the signal characteristics only on initial tests performed on a new deployed channel. It is clear that some amount of real-time monitoring of the characteristics is mandatory to provide information to the system and/or network controllers. This fact is mandatory in networks using IA-RWA algorithms.

On the other hand, it is an important requirement for an optical network, comprised of multiple point-to-point links, that the signals propagating throughout being of sufficient quality to detect. Historically, this has been achieved by the use of electrical repeaters. These convert the incoming optical signal into an electrical signal from which the base data is recovered before being used to transmit a new optical signal. This OEO conversion is undesirable when striving for high-bit-rate systems in which the conversion becomes a limiting factor. 


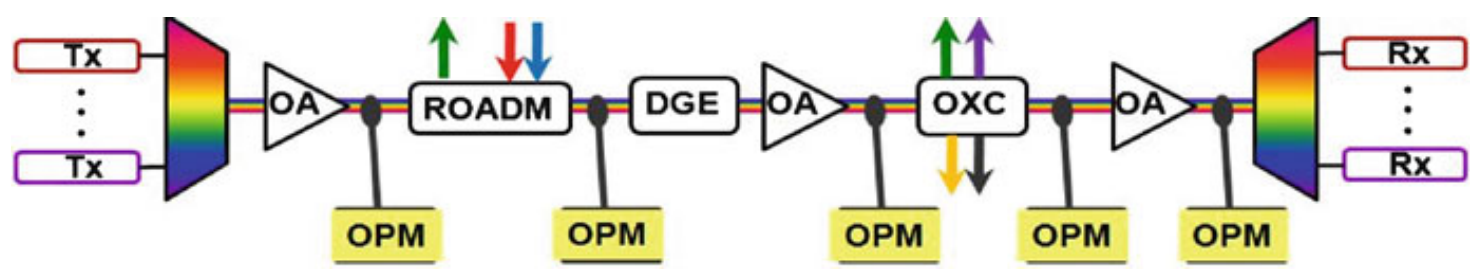

Fig. 2.14 Place of optical performance monitors $(O P M)$ in reconfigurable and dynamic alloptical network with optical amplifiers $(O A)$, reconfigurable add/drop multiplexer (ROADM), dynamic gain equaliser $(D G E)$ and optical cross-connect switches $(O X C)$

Optical amplifiers have removed the OEO conversion but added ASE noise to the optical signal. On the other hand, in future optical transport technologies of 100 Gbit/s transmission over around 1,000 km, CD has a strong effect in limiting transmission bandwidth. Signal regeneration and CD compensation in the optical domain are two approaches to solve the problem. In the case of optical regenerator, additional processing functions such as amplitude equalisation (reshaping) and temporal repositioning (retiming) of the optical pulses are developed.

Some, not exhaustive, description of monitoring, compensation and signal processing techniques are presented in the next sections, not pretending to be an exhaustive description of state of the art; but some examples to show their potential, with some specific contributions from the authors in them.

\subsubsection{Optical Performance Monitoring}

The term OPM (Chung 2008) generally refers to monitoring techniques operating at a lower level than the data protocol monitoring, which measures protocol performance information. OPM techniques include spectral (optical or electrical) and time (optical or electrical) domain techniques. Some examples of the spectral domain techniques are presented in Sect. 2.3.1.1. Section 2.3.1.2 focuses on asynchronous time-domain sampling of the photo-detected signal and Sect. 2.3.1.3 presents optical time domain reflectometry applications to OPM.

Figure 2.14 shows different strategic places for transport signal quality monitorng (Kilper et al. 2009; Bendelli et al. 2000).

Key requirements regarding OPM are: (1) small size; (2) fast and flexible measurements; (3) operation at low input power; (4) multichannel operation: monitoring of several channels in parallel or consecutively; (5) bit rate and modulation format transparency (mixed traffic can be present on the line or signal formats may change during the lifetime of the OPM) (Bendelli et al. 2000). Moreover, the OPM should be: passive, remotely configurable and low cost compared to conventional test equipment.

Depending on the type of physical parameters which are used to perform OPM, one can distinguish basic OCM and advanced signal quality monitoring (Kilper et al. 2004a). Nowadays, OCM becomes very common in WDM systems. Key parameters 


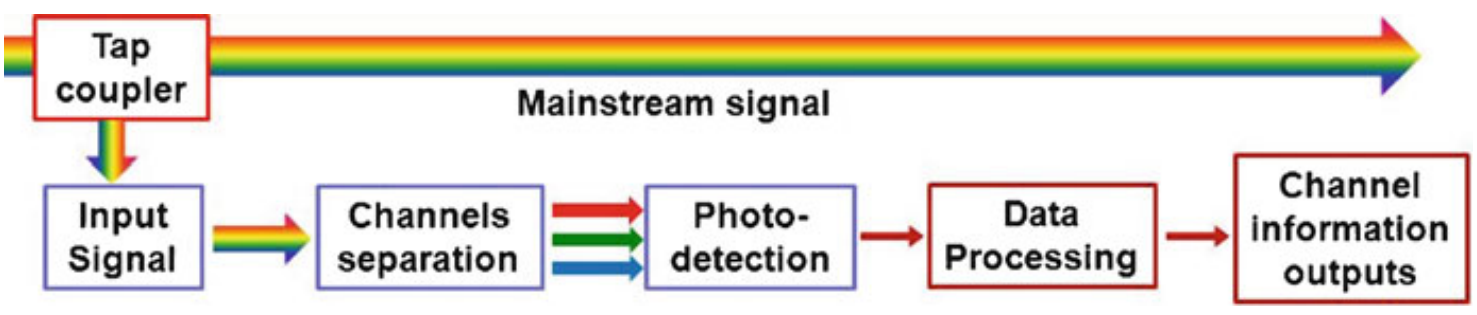

Fig. 2.15 Optical channel monitoring functional blocks

to be monitored are: channel wavelength, channel power, OSNR and their respective drifts. According to (Kirstaedter et al. 2005), the values have to be obtained every $10 \mathrm{~ms}$ for power and wavelength and $100 \mathrm{~ms}$ for optical OSNR. On the other hand, such signal distortions as in-band OSNR, accumulated CD and PMD are considered as advanced parameters which need more complex monitoring techniques.

\section{Optical Channel Monitoring}

Optical power at a given wavelength is the basic parameter for any WDM network. For monitoring purposes, a fraction (typically 1\%) of the light power is tapped from the mainstream optical signal. Then, this tapped weak signal is optically demultiplexed or filtered, in order to separate the channels, and then directed to the photodetector. Optical signal is converted to electrical signal for processing and finally channel information is transmitted to the network manager (Fig. 2.15).

A simple way to accomplish this can be using a convenient diffraction grating, such as a free space VPHG, a FBG or an AWG with a photodiode array (Pinart et al. 2005) (ENABLENCE). However, this approach is still quite expensive as it requires a large number of photodetectors to cover a wide spectral span at high resolution. Another way to monitor the WDM channels consists of using a single detector combined with one of various types of tunable filters, such as a thin-film filter, an MEMs tunable filter, a PZT-tuned Fabry-Perot filter, an acousto-optic tunable filter and a temperature-tuned etalon filter (Cahill et al. 2006). But these techniques require complex tuning mechanisms and sometimes have insufficient resolution. Nowadays, both approaches have been commercialised, and current standard OPM technology with OSA approach ensures standardised measurements according to ITU-T G.697 (ITU-T G.697). Table 2.1 presents typical specifications for this category of monitors.

The main difference between these devices is the response time, determined as the sum of scan, data processing and report times. Depending on the measurement resolution and parameters to be monitored, full scanning can take from about $10 \mathrm{~ms}$ to few hundreds of ms to complete a measurement across the entire C-band. Nevertheless, some of equipment manufactures add the OCM module to their products, such as DGE, ROADM, optical switch, etc. (LIGHTWAVE) (JSDUNPH). 
Table 2.1 Typical specifications of commercial optical channel monitors

\begin{tabular}{lll}
\hline Parameters & Value & Units \\
\hline Channel spacing & 50100 & $\mathrm{GHz}$ \\
Wavelength range & C-, L- or C + L-band & $\mathrm{nm}$ \\
Channel number (for C-band) & $>80>40$ & \\
Absolute wavelength accuracy & \pm 50 & $\mathrm{pm}$ \\
Relative wavelength accuracy & \pm 30 & $\mathrm{pm}$ \\
Dynamic range & $>30$ (typically 50) & $\mathrm{dB}$ \\
Maximum input channel power & From -10 to +5 & $\mathrm{dBm}$ \\
Absolute channel power resolution & \pm 0.5 & $\mathrm{~dB}$ \\
Relative channel power resolution & \pm 0.3 & $\mathrm{~dB}$ \\
PDL & $<0.3$ & $\mathrm{~dB}$ \\
OSNR out-of-band & $>25>28$ & $\mathrm{~dB}$ \\
OSNR accuracy & \pm 0.75 (typically \pm 1.5$)$ & $\mathrm{dB}$ \\
Scan and report time & From 10 to 1,000 & $\mathrm{~ms}$ \\
\hline
\end{tabular}
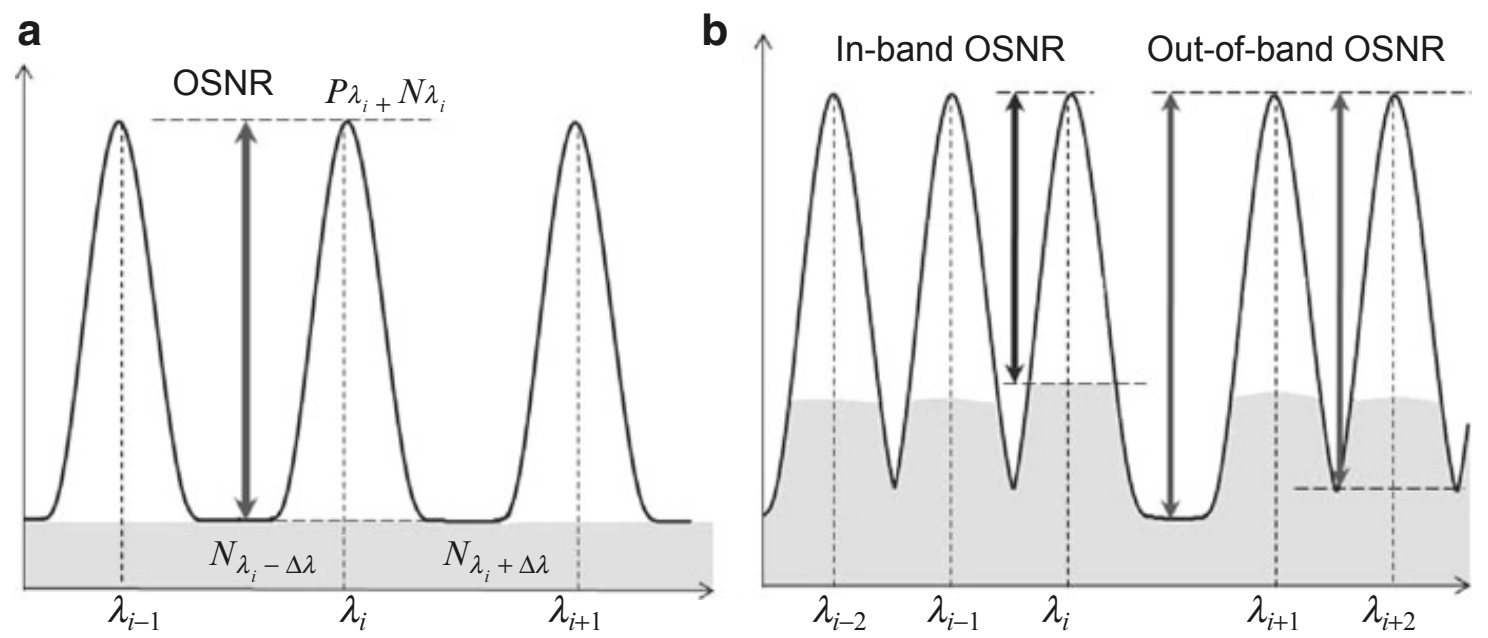

Fig. 2.16 (a) Linear interpolation method for OSNR measurements; (b) Comparison between out-of-band OSNR method and in-band OSNR method

But the real limitation of this OSA-based OPM is the optical noise measurement. For calculating OSNR, the most appropriate noise power value is that at the channel wavelength. However, with a direct spectral measurement, the noise power at the channel wavelength is included in signal power and is difficult to extract. An estimation of the channel noise power can be made by interpolating between the noise power values on both sides of the channel (Fig. 2.16a).

This assumption becomes invalid for current DWDM networks due to signal overlap from neighbouring channels, in-line filtering, spectrum broadening from non-linear effects, four wave mixing introduced noise, etc. With higher modulation rates and narrower channel spacing, the modulation sidebands from adjacent channels interfere and limit the ability to measure the noise level between channels (Fig. 2.16b). Increasing the resolution of the optical spectrum analyser does not remove this limitation. 


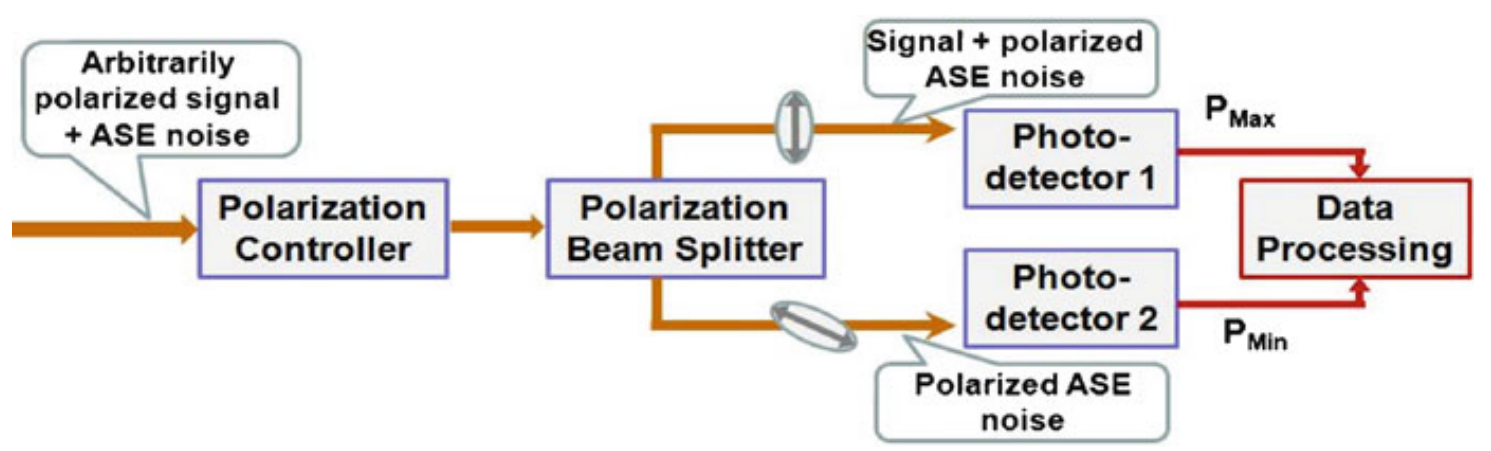

Fig. 2.17 Schematic diagram of the polarisation nulling method

Modulation tone techniques have also been used as a low-cost alternative to spectral measurements. But the principal limitation is the same: optical noise power is extrapolated from the power level adjacent to the channel (Pan et al. 2010).

As the OSNR is the key performance parameter in optical networks that predicts the bit error rate of the system, the in-band OSNR becomes essential in reconfigurable networks.

\section{In-Band OSNR Monitoring}

The challenge in this case is to discriminate the noise and the signal in the same spectral band. The polarisation nulling method overcomes some of the limitations of conventional OSA for OSNR measurement. This approach is based upon the hypothesis that an optical signal has a well-defined polarisation, while the ASE noise component is unpolarised, which allows using the polarisation extinction ratio as a measure of the OSNR (Pan et al. 2010; Kirstaedter et al. 2005; Lee et al. 2006).

As shown in Fig. 2.17, a high extinction ratio polarisation beam splitter is used to split the input signal into two arms, both being polarised in orthogonal linear states, and then detected simultaneously ( $P_{1}$ and $P_{2}$, respectively). An adjustable PC is used to find the maximum extinction of the signal when one component consists of signal and polarised noise, while the other contains only polarised noise. A measurement of the in-band OSNR will need multiple scans with different settings of the PC. The sum of $P_{1 \min }$ and $P_{2 \min }$ indicates the non-polarised in-band noise $\left(P_{\text {Noise }}\right)$, whereas for a given polarisation state of the signal, the sum of $P_{1}$ and $P_{2}$ corresponds to $\left(P_{\text {Signal }}+P_{\text {Noise }}\right)$. At the end of the measurement, the in-band OSNR values for each channel are calculated with the following equation:

$$
\mathrm{OSNR}=\frac{P_{1}+P_{2}-\left(P_{1, \min }+P_{2, \min }\right)}{\left(P_{1, \min }+P_{2, \min }\right)}
$$

Unfortunately, the performance of this technique could be affected by various polarisation effects in the transmission link. For example, it could be seriously 
deteriorated if the signal is depolarised by PMD and non-linear birefringence or the ASE noise is partially polarised due to polarisation-dependent loss.

This method has been successfully implemented in dual port optical spectrum analysers, which became recently commercially available (EXFO; JDSU).

Another method is the optical subcarrier monitoring in which each WDM channel is associated with a subcarrier (small amplitude-modulated RF frequency pilot tone) (Rossi et al. 2000). Because the tone is at a single, low frequency, it can be easily generated and processed using conventional electronics. The average power in these tones will be proportional to the average optical power in the channel, and the aggregate WDM optical signal on the line can be detected; the tones of all the channels will appear in the RF power spectrum in much the same way they would appear in the optical spectrum. Thus, optical parameters can be monitored without using the expensive optical devices, such as tunable optical filter or diffraction grating. The electrical CNR of the subcarrier will be determined and the OSNR is obtained through a mathematical relationship with CNR. This method has an advantage in that it involves monitoring on the actual data signal as it has propagated along the impairment path of the signal itself and can be implemented with narrowband electronics. Moreover, the monitoring of RF tones can be used for measuring the accumulation of CD and PMD on a digital signal (Rossi et al. 2000).

The major drawbacks of this technique are that the AM tone and data could interfere with each other and cause deleterious effects. Thus, the amplitude of the pilot tone should be large enough to discern the tone signals from the noise-like random data, but small enough not to induce a significant degradation in the receiver sensitivity for data.

MZI method is based on the difference of behaviour between a coherent signal, which is able to interfere at the output of the interferometer, and non-coherent ASE noise. By adjusting the path difference between the two arms, the maximum (constructive interference) and minimum (destructive interference) output powers are obtained, and OSNR can be derived while it is proportional to the ratio $P_{\text {const }} / P_{\text {dest }}$. With increasing ASE power (i.e. decreasing ONSR), $P_{\text {dest }}$ increases faster than $P_{\text {const }}$ because of the random phase of the noise (Liu et al. 2006).

The most promising results was obtain with a 1/4-bit delay method. Since the phase relationship between successive bits is not important, the method is applicable to multiple modulation formats (Lizé et al. 2007).

Uncorrelated beat noise can also be used for OSNR monitoring (Chen et al. 2005). This method is compatible with different modulation formats, independent of the pattern length and insensitive to PMD.

\section{Chromatic Dispersion and Polarisation-Mode Dispersion Monitoring}

We give here a short description of existent technologies for real-time CD and PMD monitoring which are summarised in Pan et al. 2010. 
Firstly, monitoring techniques based on RF tone (conversion of a phase modulated signal into an amplitude-modulated one by inserting a subcarrier at the transmitter) are relatively simple and quite fast but may require transmitter modification.

The RF clock techniques are based upon the same concepts as the RF pilot tones techniques, with a monitored frequency corresponding to the bit rate. The clock power detection technique has been used as CD and PMD monitors, whereas the technique based on phase detection is used for $\mathrm{CD}$ monitoring only. The main advantage of the clock techniques is the absence of modification of the transmitter; however, they are potentially expensive (single channel operation).

\section{Impact of New Modulation Formats}

The techniques presented in this section have been first introduced to monitor OOK (mostly NRZ) $10 \mathrm{Gbit} / \mathrm{s}$ signals. Most of them can be applied to more advanced modulation formats that are envisioned for 40 or $100 \mathrm{Gbit} / \mathrm{s}$ transmission. This trend towards more complex modulation schemes could, however, have an impact on the deployment of OPM functions. There will still remain a need for the monitoring of the basic parameters (power, OSNR) of multiplexed channels. On the other hand, the high spectral efficiency and related robustness against DC and PMD of these modulations could reduce the need of in-line monitoring of DC and PMD. For instance, it has been shown that CO-OFDM signal is robust against PMD and tolerates a chromatic dispersion equivalent to $3,000 \mathrm{~km}$ standard singlemode fibre. Moreover, these modulation formats involve advanced signal processing algorithms in the receivers which can provide information about the impairments experienced by the incoming signals. In (Shieh et al. 2007), OCE through receiver signal processing is proposed as one approach to optical performance monitoring. Most importantly, performance monitoring by OCE is basically free because it is embedded as a part of the intrinsic receiver signal processing. Such a monitoring device could also be placed anywhere in the network without concern about the large residual chromatic dispersion of the monitored signal. Cost and standardisation issues will be determinant to select among the different per-channel monitoring techniques: optical and/or RF spectrum analysis, digital signal processing (which implies clock recovery) and asynchronous sampling which will be discussed in the next section.

\subsubsection{Asynchronous Performance Monitoring}

In the previous section, we introduced several techniques for the monitoring of a WDM channel. These techniques are based on the analysis of the optical or electrical spectrum of a group of channels or of a single channel, where some extra monitoring signals (e.g. pilot tones) have been possibly added. The present section is dedicated to time-domain monitoring techniques, which involve the sampling of the channel 
Fig. 2.18 Representation of the sampling of a signal $x(t)$ with a sampling period TS and a sampling window defined by the function

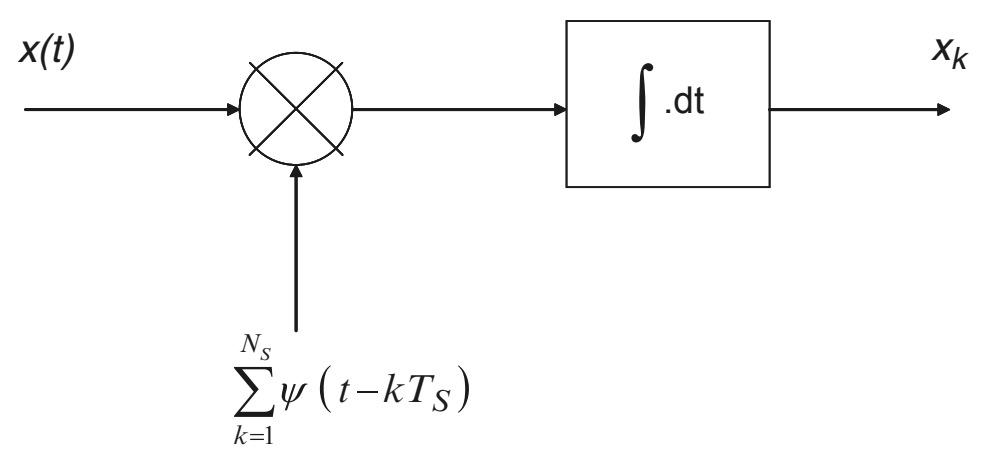

to be monitored and a statistical analysis of the acquired samples. For all these techniques, it is assumed that the channel to be monitored has been isolated from the rest of the optical comb.

We will first consider an amplitude-modulated binary digital signal $x(t)$, with a bit duration TB and bit frequency $f_{\mathrm{B}}=1 / T_{\mathrm{B}}$. Figure 2.18 provides a diagram of a simplified sampling system where $x(t)$ is multiplied by a train of periodic sampling pulses centred in the sampling instants $k \cdot T_{\mathrm{S}}, \mathrm{w}$ h e $\mathrm{r}$ e $T_{\mathrm{S}}$ is the sampling period (and $f_{\mathrm{S}}$ the sampling frequency). Each sampling pulse $\psi(t)$ has duration $T_{\text {res }}$ and is generally assumed to be a gate function. The multiplication can be performed either in the optical domain (for instance, by using sum-frequency generation in a non-linear crystal (Shake et al. 1998)) or, more commonly, in the electrical domain by gating the photo-detected signal (e.g. in (Mueller 1998)). A set of $N_{\mathrm{S}}$ samples is acquired. $N_{\mathrm{S}}$ should be high enough to contain the entire statistics of the signal.

Let us assume, that $f_{\mathrm{S}}=\frac{n}{m} f_{\mathrm{B}}+f_{\text {off, }}$, where $n$ and $m$ are two natural numbers which minimise $\left|f_{\mathrm{S}}-\frac{n}{m} f_{\mathrm{B}}\right|$ and $f_{\text {off }}$, is the offset frequency. In the conventional synchronous sampling technique, $f_{\mathrm{S}}$ is synchronised with $f_{\mathrm{S}}$ in order to satisfy (Shake et al. 2004):

$$
T_{\text {step }}=\frac{1}{f_{\mathrm{S}}}-\frac{1}{\left(\frac{n}{m}\right) f_{\mathrm{B}}}=\frac{1}{p f_{\mathrm{B}}}
$$

where $T_{\text {step }}$ is the interval between the $p$ sampling time positions inside the bit duration. This implies a clock recovery of $f_{\mathrm{B}}$. The above relationship determines the offset frequency for synchronous sampling.

In the case of asynchronous sampling, the offset frequency does not satisfy (2); thus, if $N_{\mathrm{S}}$ is high enough, the sampling instants will be uniformly spread across the entire bit period. Figure 2.19 shows an example of both synchronous and asynchronous histograms and the corresponding eye diagram.

An example of typical asynchronous sampling parameters for a $10 \mathrm{Gbit} / \mathrm{s} \mathrm{NRZ} \mathrm{or} \mathrm{RZ}$ signal, taken from (Shake et al. 1998), is $f_{\mathrm{S}}=\left(f_{\mathrm{B}} / 1,024\right)-10 \mathrm{kHz} \approx 9.7 \mathrm{MHz}, T_{\text {res }}=$ 1 . $\mathrm{s}$ and $\mathrm{NS} \approx 1.5104$. The $T_{\text {res }}$ value is generally fixed, much shorter than the bit period, in order to avoid loss of information due to averaging effects. However, by noting that the averaging effect mostly concerns the noise, it is possible to relax this constraint and use sampling durations close to the half bit period. This value 

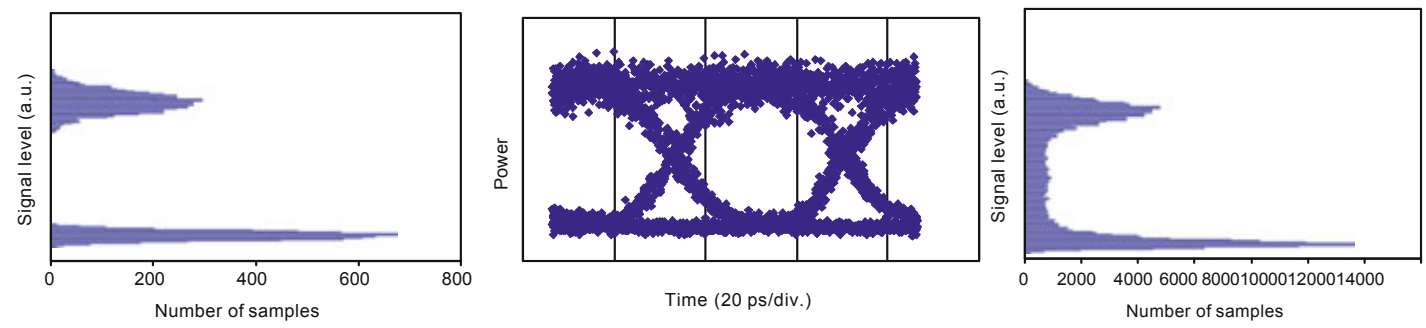

Fig. 2.19 Eye diagram example (centre) of a $10 \mathrm{Gbit} / \mathrm{s} \mathrm{NRZ} \mathrm{signal} \mathrm{with} \mathrm{associated}$ synchronous (left) and asynchronous (right) histograms

may even nearly reach $T_{\mathrm{B}}$ if the sample processing takes into account inter-symbol interference (Luis et al. 2004) to the expense of an increased processing complexity. The main motivation for asynchronous sampling is the absence of clock recovery which makes it less expensive than synchronous sampling and enables it to work at a wide variety of bit rates. This is a clear advantage in the context of transparent optical networks, but several issues need to be solved in order to apply it as a monitoring technique. In particular, it should allow identifying the strength and the origin of signal perturbation.

Since the early proposals of asynchronous performance monitoring (Shake et al. 1998; Mueller 1998), different studies have been carried to address this issue, especially by deducing the Q-factor from the asynchronous histogram. A simple analysis can be provided for NRZ coding and negligible inter-symbol interference (Luis et al. 2004). At a fixed timing phase $t_{0}, Q\left(t_{0}\right)$ is defined by:

$$
Q\left(t_{0}\right)=\left|\mu_{1}\left(t_{0}\right)-\mu_{0}\left(t_{0}\right)\right| /\left|\sigma_{1}\left(t_{0}\right)+\sigma_{0}\left(t_{0}\right)\right|
$$

where $\mu_{i}\left(t_{0}\right)$ and $\sigma_{i}\left(t_{0}\right)$ are the mean and standard deviation of the mark(1) and space $(0)$ levels at $t_{0}$, respectively. If the choice of $t_{0}$ corresponds to the optimum decision time, $Q\left(t_{0}\right)$ reduces to the usual Q-factor, which (assuming Gaussian distributions of mark and space amplitudes) is linked to the BER by:

$$
\mathrm{BER}=\frac{1}{2} \operatorname{erfc}\left(\frac{Q}{\sqrt{2}}\right)
$$

When performing asynchronous sampling, it is only possible to measure the average Q-factor $\left(Q_{\text {ave }}\right)$, defined by:

$$
Q_{\text {ave }}=\left|\mu_{1, \text { ave }}-\mu_{0, \text { ave }}\right| /\left|\sigma_{1, \text { ave }}+\sigma_{0, \text { ave }}\right|
$$

where $\mu_{i \text { ave }}$ and $\sigma_{I \text {,ave }}$ are the mean and standard deviation of the mark(1) and space $(0)$ of all sampled data, respectively.

To get useful information from asynchronous sampling, one needs to derive a relationship between $Q_{\text {ave }}$ and $Q$. It is quite intuitive that $Q_{\text {ave }}$ will be smaller than $Q$ because the data obtained by asynchronous sampling include unwanted cross-point 

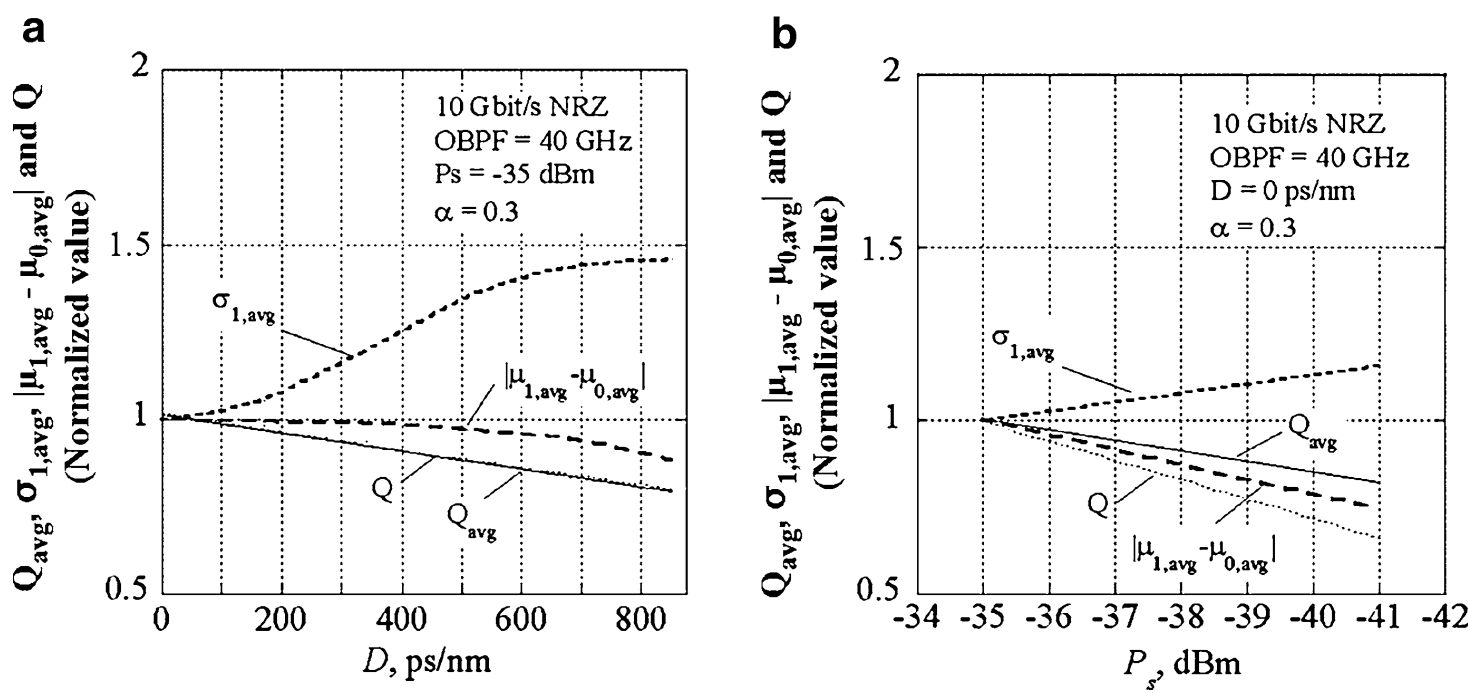

Fig. 2.20 Chromatic dispersion dependence (a) and OSNR (b) dependence of $\sigma_{1 \text { ave }}$, $\left|\mu_{1 \text {.ave }}-\mu_{0 \text { ave }}\right|$, and $Q_{\text {ave }}$ for a $10 \mathrm{Gbit} / \mathrm{s} \mathrm{NRZ}$ optical signal with a $25 \mathrm{ps}$ rise time and $T_{\text {res }}=1 / 64$ $T_{\mathrm{B}}, N_{\mathrm{S}}=16,384, B_{\mathrm{opt}}=40 \mathrm{GHz}, B_{\mathrm{el}}=7.5 \mathrm{GHz}$ and $\alpha=0.3$ (๑) 2003 IEEE, after Ref (Shake 2003))

data in the eye diagram. The effect of these cross-points can be limited by using a threshold $\alpha(0<\alpha<0.5)$ in order to define $\mu_{\text {th } 0}=\mu_{0 \text {,ave }}+\alpha\left|\mu_{1, \text { ave }}-\mu_{0, \text { ave }}\right|$ and $\mu_{\text {th1 }}=\mu_{1, \text { ave }}-\alpha\left|\mu_{1, \text { ave }}-\mu_{0, \text { ave }}\right|$ and discarding the values comprised between $\mu \mathrm{th}_{0}$ and $\mu \mathrm{th}_{1}$. In practice, values of $\alpha$ between 0.1 and 0.5 have been used and result in linear relationship between $Q_{\text {ave }}$ and $Q$, when $13 \mathrm{~dB}<Q<20 \mathrm{~dB}$ (for the sake of simplicity, the same notation is used for the linear and decibel expressions of $Q$ ). The actual slope of this curve depends mainly on the $B_{\text {opt }} / f_{\mathrm{B}}$ and $B_{\mathrm{el}} / f_{\mathrm{B}}$ ratios where $B_{\mathrm{opt}}$ and $B_{\mathrm{el}}$ are the optical and electrical bandwidths of the sampling system, respectively (Shake and Takara 2002). Reference (Luis et al. 2004) provides a model based on an equivalent filter $h_{\mathrm{eq}}(t)=\psi(-t)$ (where $\psi$ has been introduced in Fig. 2.18), which confirms this linear dependence for IM/DD signals without inter-symbol interference, when the spontaneous-spontaneous beat noise can be neglected.

Beyond the estimation of the Q-factor, the analysis of asynchronous histograms enables to detect signal degradations due to noise, crosstalk or pulse distortion. Indeed, these effects have different impacts on the various $\mu$ and $\sigma$ values. ASE noise will reduce $\left|\mu_{1, \text { ave }}-\mu_{0, \text { ave }}\right|$ and increase both $\sigma_{0, \text { ave }}$ and $\sigma_{1, \text { ave }}$, while crosstalk will mainly increase $\sigma 1$,ave; finally, chromatic dispersion will have a more pronounced impact on $\sigma 1$,ave than on $\left|\mu_{1 \text {,ave }}-\mu_{0, \text { ave }}\right|$. All these perturbations yield noticeable modifications of the asynchronous histograms for B.E.R. $>10^{-12}$. A detailed analysis of the evolution of the histograms in the presence of both noise and dispersion can be found in Shake (2003). Figure 2.20, reproduced from (Shake 2003), shows an example of the evolution of $\sigma_{1, \text { ave }}$ and $\left|\mu_{1, \text { ave }}-\mu_{0, \text { ave }}\right|$ as a function of received power and line chromatic dispersion. 


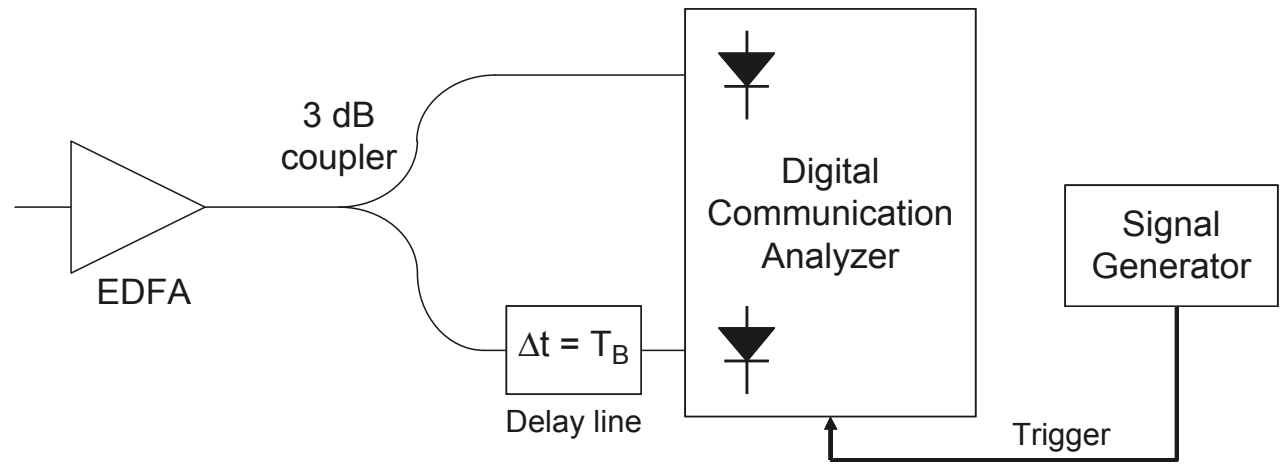

Fig. 2.21 Two-tap asynchronous sampling set-up with a separation between sample pairs $=T_{\mathrm{B}}$

Nevertheless, the interpretation of conventional asynchronous histograms re-mains difficult in the presence of simultaneous transmission impairments. In order to solve this issue, a two-tap sampling technique has been proposed (Anderson et al. 2006; Dods 2006).

In this technique, the asynchronous samples are collected by pairs $\left(x_{k}, y_{k}\right)$, with $x_{k}$ $+1=x_{k}+T_{\mathrm{S}}$ and $y_{k}=x_{k}+\left(T_{\mathrm{B}} / p\right)$ where usually $p=1,2$ or 4 (Fig. 2.21). The two-tap histogram is obtained by plotting the $N_{\mathrm{S}}$ points with coordinates $\left(x_{k}, y_{k}\right)$.

For an NRZ modulation with $p=1$, the ideal figure (without impairments) represents the edges of a square with its secondary diagonal: the edges correspond to $001,011,110$ and 100 bit sequences, while the diagonal corresponds to the 010 and 101 sequences. In contrast to conventional asynchronous or synchronous sampling techniques, this method captures information about the distribution of closely spaced samples, which makes it particularly suited to the monitoring of pulse distortion.

The ability of two-tap sampling to provide information about simultaneous impairments is illustrated in Fig. 2.22 which shows the impact on the histograms of up to four combined impairments, for delay values of $T_{\mathrm{B}}, T_{\mathrm{B}} / 2$ and $T_{\mathrm{B}} / 4$. The different figures can generally be well distinguished, except for the limited effect of PMD on the histogram with $T_{\mathrm{B}}$ delay.

The analysis of these two-tap histograms can be performed automatically with estimation techniques used in pattern recognition (Anderson et al. 2006), including three-layer artificial neural networks (Jargon et al. 2009). Until recently, most of the work on asynchronous performance monitoring was carried on OOK data. New generations of high capacity optical transmission systems will likely involve more complex modulation schemes and coherent detection. This could increase the complexity of the sampled patterns and make more difficult a quantitative analysis of the different impairments. However, recent results suggest that the two-tap asynchronous sampling technique can be applied to various PSK modulation schemes. An example is given on Fig. 2.23, which shows the results of the processing by a three-layer artificial neural network of $40 \mathrm{Gbit} / \mathrm{s}$ RZ-BPSK data after two-tap asynchronous sampling, with $T_{\mathrm{B}} / 2$ delay. Both direct and balanced 


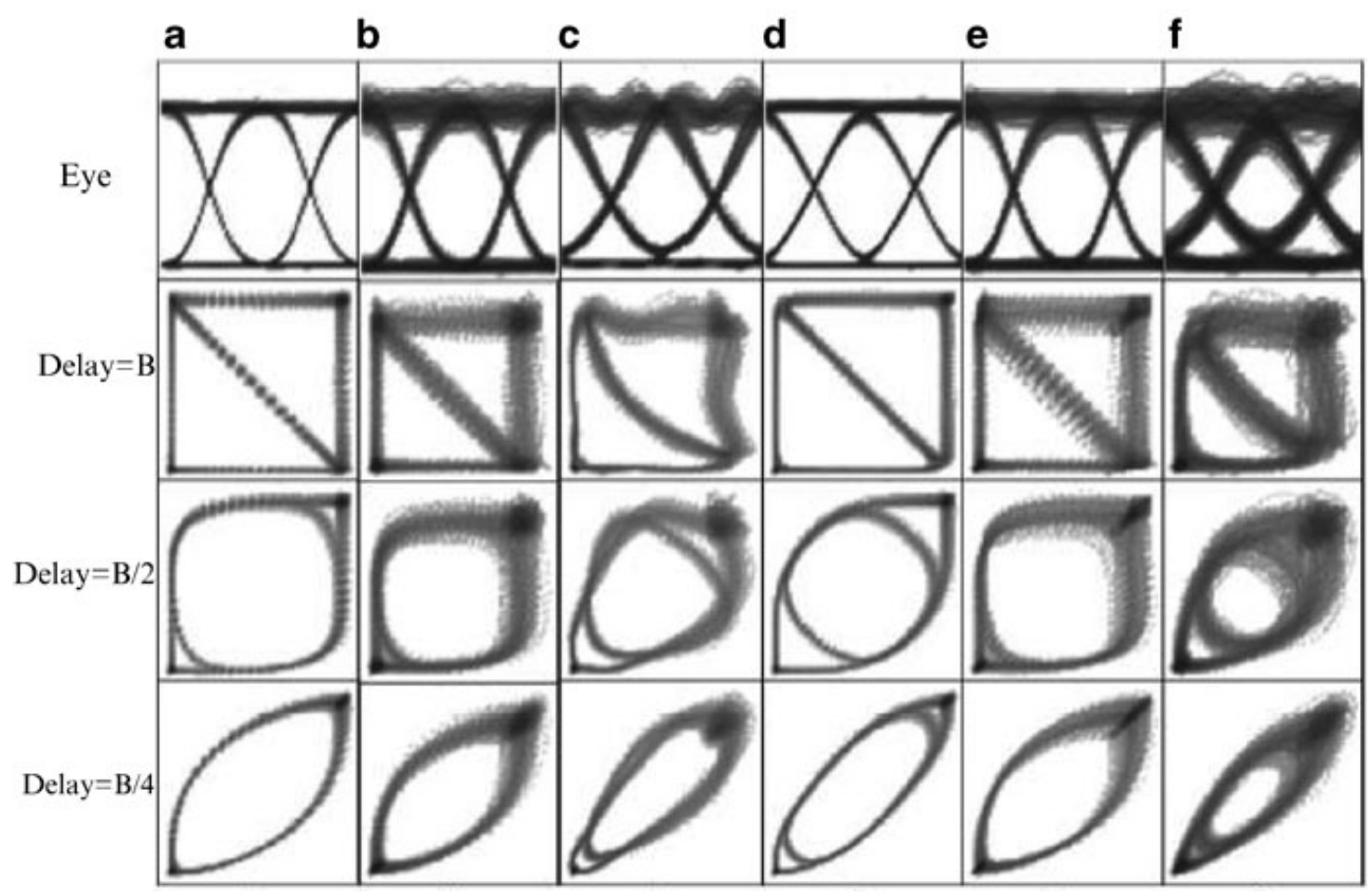

Fig. 2.22 Eye diagram and two-tap plots for delay $=T_{\mathrm{B}}, T_{\mathrm{B}} / 2$ and $T_{\mathrm{B}} / 4$ for (a) $\operatorname{OSNR}=35 \mathrm{~dB}$ and no impairment, (b) OSNR $=25 \mathrm{~dB}$ and no impairment, (c) OSNR $=35 \mathrm{~dB}$ and $D=800 \mathrm{ps} / \mathrm{nm},(\mathbf{d})$ OSNR $=35 \mathrm{~dB}$ and $\mathrm{PMD}=40 \mathrm{ps}$, (e) OSNR $=35 \mathrm{~dB}$ and interferometric crosstalk $=-25 \mathrm{~dB}$, (f) O S N R $=25 \mathrm{~dB}, D=800 \mathrm{ps} / \mathrm{nm}, \mathrm{PMD}=40 \mathrm{ps}$ and crosstalk $=-25$ dB (@ 2006 IEEE, after Ref (Anderson et al. 2006))

a
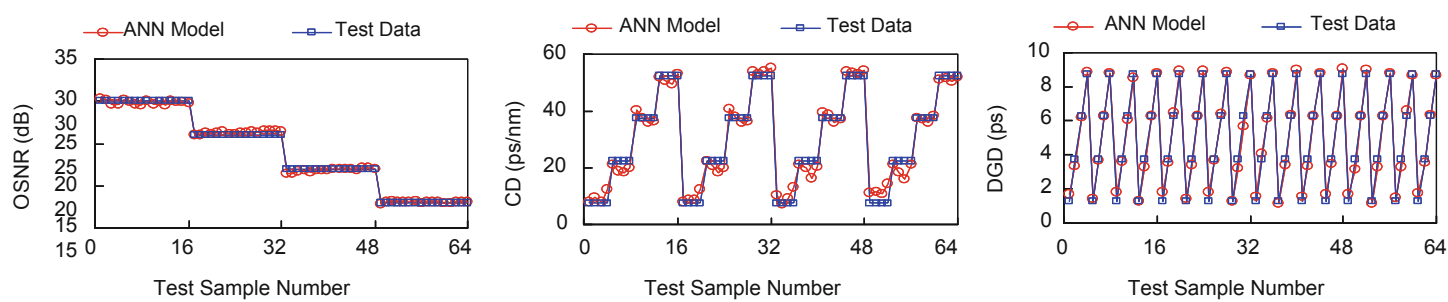

40-Gbit/s RZ-BPSK ANN testing results from balanced detection.

b
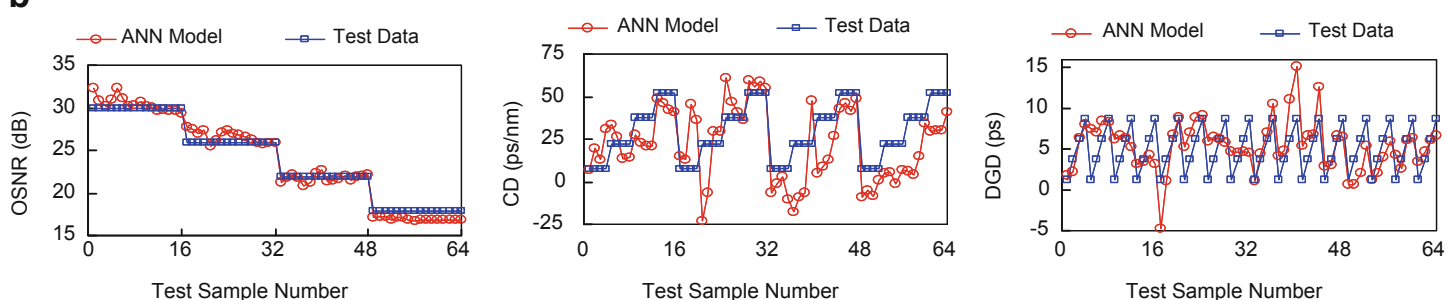

40-Gbit/s RZ-BPSK ANN testing results from direct detection.

Fig. 2.23 Comparison of testing and artificial neural networks - model data for a $40 \mathrm{Gbit} / \mathrm{s}$ RZ-BPSK channel (C 2009 VDE Verlag after Ref. (Wu et al. 2009)) 
detection schemes are compared. The later provide a much more accurate prediction of the impairments, especially for CD and PMD.

This approach can be extended to more complex modulation schemes: asynchronously generated constellation patterns have been used to estimate the amount of CD and DGD of DQPSK signals (Arbab et al. 2009).

In conclusion, asynchronous sampling monitoring can provide as much useful information on the impairments experienced by an optical signal as the conventional eye-diagram monitoring, but without necessity of a clock recovery. This method is also quite sensitive as it can detect impairment levels responsible of a rough (before FEC) $10^{-12}$ BER in a few milliseconds, much faster than direct BER measurement. The simple one-tap technique allows measuring an averaged Q-factor and is well suited to NRZ or RZ modulations, when one type of impairment is likely to predominate. It is, to a large extent, bit-rate independent, even though the accuracy of its prediction can be improved when the sampling parameters are adapted to the bit rate. The two-tap technique is, by nature, bit-rate dependent, but has proven to be much more powerful to provide reliable results by using, e.g. artificial neural network-based algorithm, to process the samples, in the presence of simultaneous impairments, and it offers a good potential for operation with advanced modulation schemes.

\subsubsection{Infrastructure Monitoring (OTDR)}

Installation of PONs as a main choice of the operators has been accelerating mainly due to their future proof nature and lower OPEX.

Even though PON outside plant (OSP) should be sustainable over the expected lifetime of the system, there is still room for the operators to save significant amount of OPEX using effective and easy preventive maintenance of the physical infrastructure. In today's PON systems, the physical infrastructure is usually not entirely visible to the NMS. As a direct consequence, a physical failure cannot be detected before creating service outage in upper layers which in turn may lead to tremendous loss in business for the operators. We can mention not only direct financial losses due to service interruption but also indirect financial losses due to bad reputation. These arguments have been gaining importance as the warranty on the quality of the infrastructure becomes a deciding factor in the strongly competitive marketplace.

The convergence between transport and access networks with more complex hybrid topologies and high bit rates $(10 \mathrm{Gbit} / \mathrm{s})$ over longer reach/higher split options gives rise to capacity increase and make a network failure more disastrous. The aim of preventive maintenance is to detect any kind of deterioration in the network that can cause suspended services and to localise these faults in order to avoid specially trained people deployed with dedicated and often expensive equipments for troubleshooting. PON infrastructure does not only suffer from accidental damages and environmental effects (e.g. water penetration in splice closures) but are also subject to a lot of changes after the network is installed and activated. As an 
example, the optical access network may not be initially fully loaded; subscribers would be turned up, possibly over an extended period of time (Frigo et al. 2004). Hence, network operators should continuously be aware if a change noticed by its monitoring system is service-oriented or indeed a fault. In addition to that, it is crucial to discriminate the faults (accidental interruptions) from attacks (intentional interruptions) results in a strengthening of relations between optical maintenance functions and the security management. All arguments mentioned above mean that the existing maintenance methods need to be updated making the monitoring in PONs an active research area.

The number of scientific publications has significantly increased in the last few years. Authors propose different approaches which are addressing some of the challenges of PON monitoring. Still, there are no standardised and mature monitoring methods. Ideal optical monitoring framework in PONs has the following general requirements (Yüksel et al. 2008):

- It should provide continuous, remote, automatic, and cost-effective supervision of the physical layer.

- It should provide rapid and accurate detection of performance degradation as well as service disruption.

- It should unambiguously provide failure source location.

- The testing should not affect normal data transmission (non-intrusive testing).

- It should distinguish between a failure in the end users' own equipment and a failure in the operator's network. Monitoring results should be conveyed to the NMS and evaluated here in detail enabling preventive countermeasures (like protection and restoration, isolation of attacking port...).

- It should be interoperable with many network variants (bit rate, protocol ...).

The most common maintenance tool employed for troubleshooting in long-haul, point-to-point fibre-optic links is an optical time domain reflectometer (OTDR). However, implementation of OTDRs into PONs brings some testing challenges which are: the lack of dynamic range to monitor the infrastructure after the splitter, a long measurement time due to averaging necessary to obtain an OTDR trace and repetition of the measurement on large number of ONTs, and the reflection deadzone that makes it impossible to distinguish the monitoring reflection peaks from two nearly located ONTs. Beside these general considerations, one of the main technical issues on maintenance in PON system is known as point-to-multipoint problem. In the PONs, the OTDR pulses launched into the fibre are passively split and propagate simultaneously in every branch after the splitting point. As a result, the backreflected and backscattered light signals from each branch add up together to form a "global" or composite trace that makes the interpretation of the OTDR trace from the $\mathrm{CO}$ a difficult task. Looking at the global trace without any additional information, one cannot identify the faulty branch. The fault quantisation is another problem; in order to determine the "real loss" or "real return loss" of a fault, some further calculations are needed based on the network's parameters, such as power levels measured during installation, splitting ratio and splitting insertion losses (Wuilmart et al. 1996). 


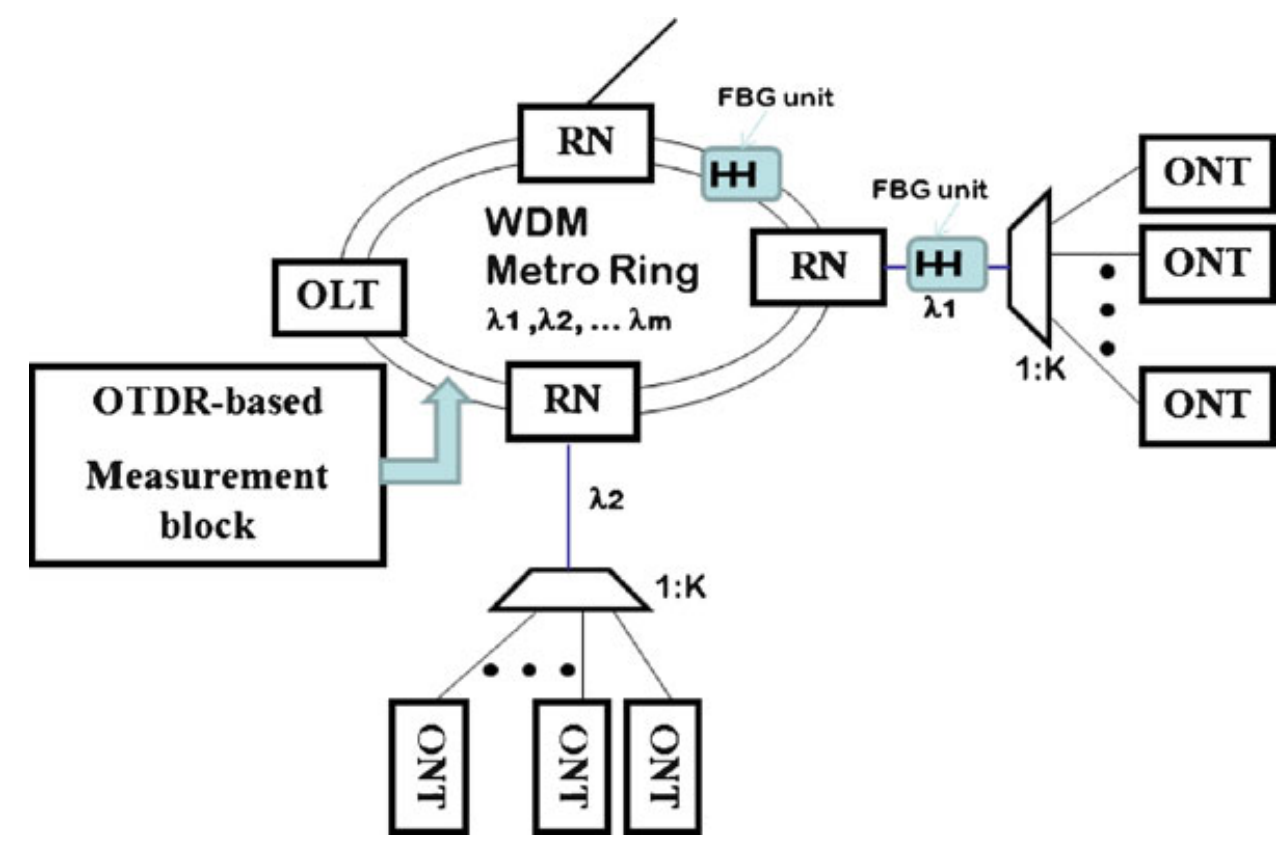

Fig. 2.24 Ring-tree PON scheme with the proposed monitoring method

When converged metro/access network topologies are considered, guarantying the reliability in these networks might be even more crucial for the operators as the architecture becomes more complex, and the first expected aim is to transport high capacity services to business customers.

In particular, the combination of WDM bidirectional rings and TDM access trees as proposed in the framework of ISTSARDANA requires an elaborated monitoring approach. It is obvious that diagnostic requirements for such a converged network are different from that of classical TDM-PONs. A conventional OTDR which operates at a single wavelength is not suitable to probe branches beyond the wavelength selective component at the $\mathrm{RN}$ which realises the add/drop functionality to assign a fixed wavelength to each TDM access tree. Hence, OTDR must have wavelength tuning capability.

A new method for monitoring such a complex hybrid topology was studied in the framework of SARDANA project (Militello et al. 2009). This method is based on a tunable OTDR and reference reflections created by FBGs.

Figure 2.24 shows the schematic of the combined ring/PON topology implementing the proposed monitoring system. A tunable OTDR is used at the OLT side and interrogates the FBGs at different central wavelengths located in the ring or the access parts of the network. For each OTDR measurement, wavelength is adjusted so that one or a group of FBGs creates reference reflection peaks on the OTDR trace which are used to check the integrity of the network.

As represented in Fig. 2.25, tunable OTDR can be implemented by using a commercially available OTDR and a wavelength conversion system (WCS). WCS includes two optical circulators ( $\mathrm{C} 1$ and $\mathrm{C} 2$ in Fig. 2.25), a TLS and an optical/electrical $(\mathrm{O} / \mathrm{E})$ converter. The optical pulses emitted by the OTDR are 


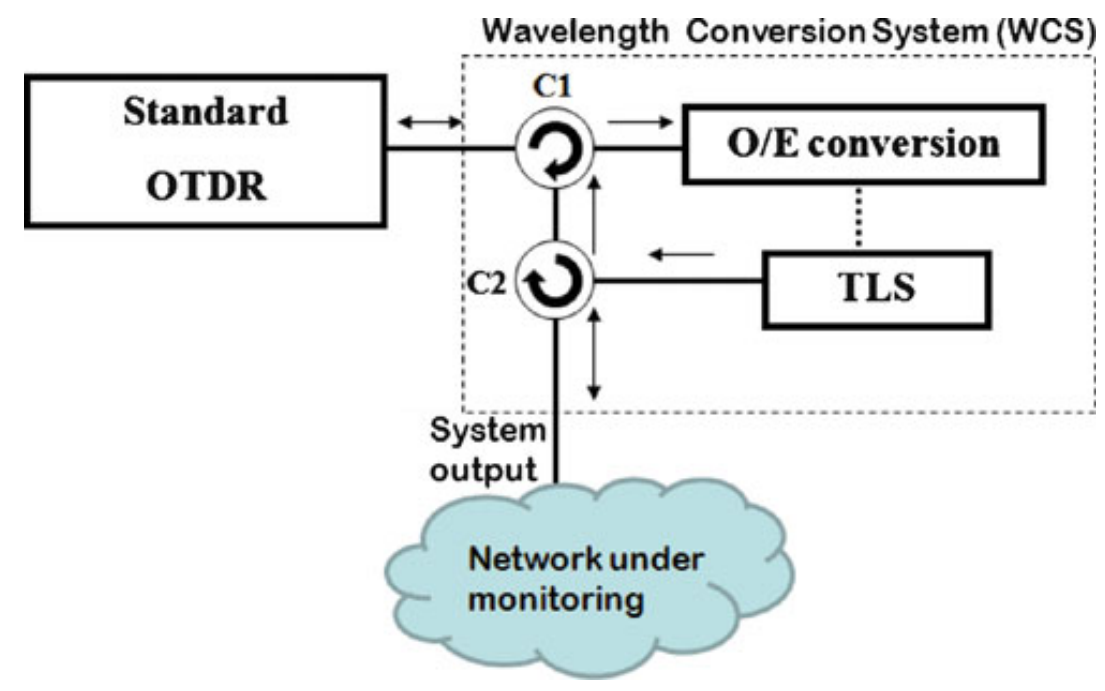

Fig. 2.25 Implementation of tunable OTDR

directed onto $\mathrm{O} / \mathrm{E}$ converter via a first circulator $(\mathrm{C} 1)$. The electrical pulses obtained at the output of $\mathrm{O} / \mathrm{E}$ converter are amplified and modulate the optical power emitted by the TLS. As a consequence, optical pulses at a desired wavelength are produced at the OTDR repetition rate (with a certain pulse delay) and directed into the network. The standard OTDR receives the reflected and backscattered signals via the two circulators $\mathrm{C} 2$ and $\mathrm{C} 1$ and stores the associated trace.

Monitoring results for an example scenario are shown in Fig. 2.26. I $n$ t h i s example case, two FBGs with central wavelengths of $1557.36 \mathrm{~nm}$ and $1560.61 \mathrm{~nm}$ are placed respectively into the ring and one PON section of the network. The OTDR trace when TLS generates pulses at 1,550 nm (Fig. 2.26a) shows reflection peaks initially present in the network (e.g. connectors). FBGs are not involved in this trace as $1,550 \mathrm{~nm}$ is out of the reflection bands of the FBGs. In Fig. 2.26b, the wavelength of the TLS is set to $1557.3 \mathrm{~nm}$ where the FBG in the ring (placed about $34 \mathrm{~km}$ from the OTDR) creates a high intensity peak. A non-assigned wavelength in the WDM-ring should be chosen to test this part of the network. Then, TLS wavelength is set to $1560.6 \mathrm{~nm}$ to detect the reflection peak due to the second FBG placed in the feeder line of a tree PON connected to the ring through the RN. The optical pulse at the TLS output is shown in Fig. 2.26d.

Apart from the solution based on OTDR, other monitoring solutions based on OFDR recently appeared in the literature as an alternative approach (Zou et al. 2007; Effenberger 2008). A new monitoring method for PONs using an OFDR at the OLT and interferometer units (IF-unit) at the ONTs/ONUs is under study. Each IF-unit includes a uniform FBG and creates a beat term (a peak) on the OFDR trace which is used to check the integrity of the corresponding branch. Analysing the beat terms of all branches allows an easy distinction of the faulty branch after the splitter. In addition to the easy determination of the faulty branch, the system directly measuresthe temperature variation in the network terminals, such as ONU/ ONT, fibre distribution hub or network access terminals.Temperature measurement is realised by using the temperature sensitivity of the FBG's spectrum 
a

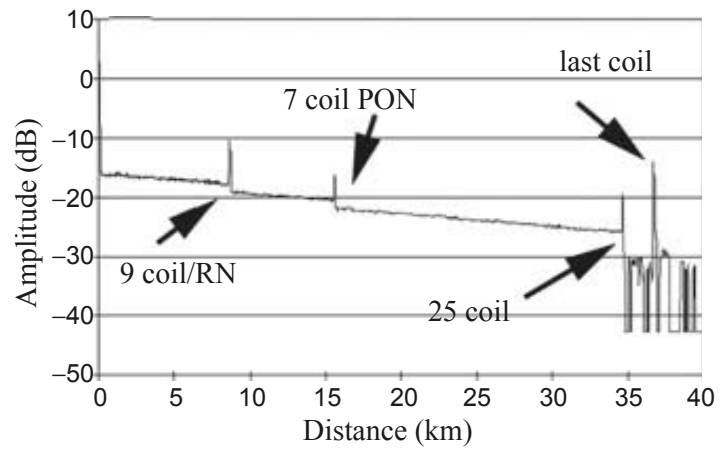

C

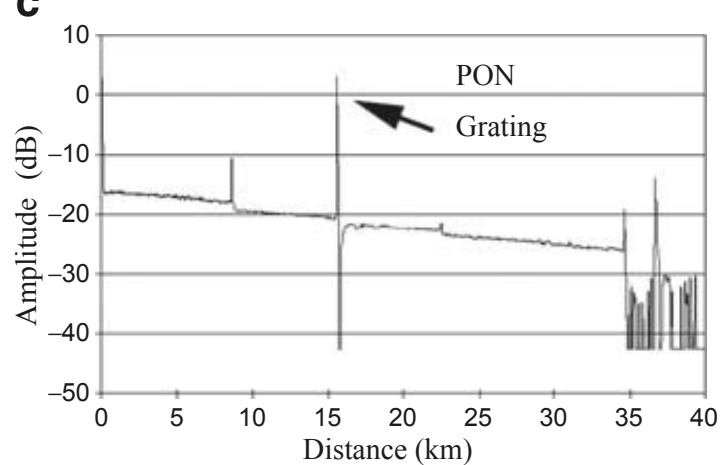

b

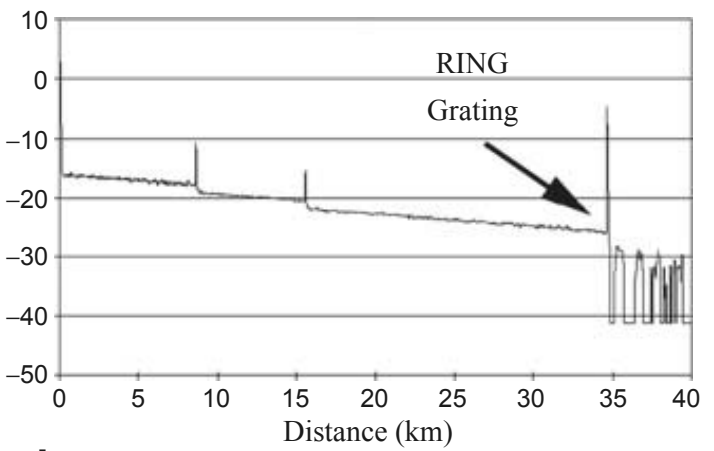

d

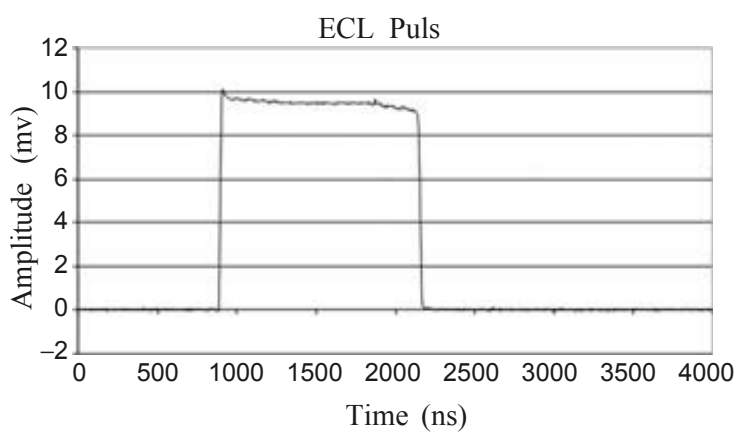

Fig. 2.26 (a) Monitoring trace at 1,550 nm, (b) monitoring trace at 1,557.3 nm, (c) monitoring trace at $1,560.6 \mathrm{~nm},(\mathbf{d})$ optical pulse at the TLS output

inside the IF-units. In this method, simple signal processing steps are applied on the OFDR trace to deduce the Bragg wavelength shift of each FBG located in each IF-unit. This information in turn gives the temperature evolution of interferometer device's position (Yuksel et al. 2010).

\subsection{Signal Processing and Compensation}

\subsubsection{Optical CD Compensation}

$\mathrm{CD}$ is an intra-channel degradation effect that takes place as a short light pulse propagates along an optical fibre, and it can be one of the main restrictions for optical digital transmission systems. CD is due to the dispersion property of the fibre, that is, the fact that its index of refraction, and thus the light velocity, varies as a function of wavelength. Short pulses are not monochromatic but rather have a certain spectral bandwidth; the shorter the pulse, the wider its spectrum and thus the stronger the temporal broadening due to $\mathrm{CD}$. This temporal broadening is also proportional to the length of the fibre span, so the $\mathrm{CD}$ becomes larger as the length of the fibre increases. In the near future, the performance of optical transport technologies such as optical Ethernet, SONET/SDH, CWDM, DWDM and OTN/ASON is expected to reach the range of $100 \mathrm{Gbit} / \mathrm{s}$ transmission over 


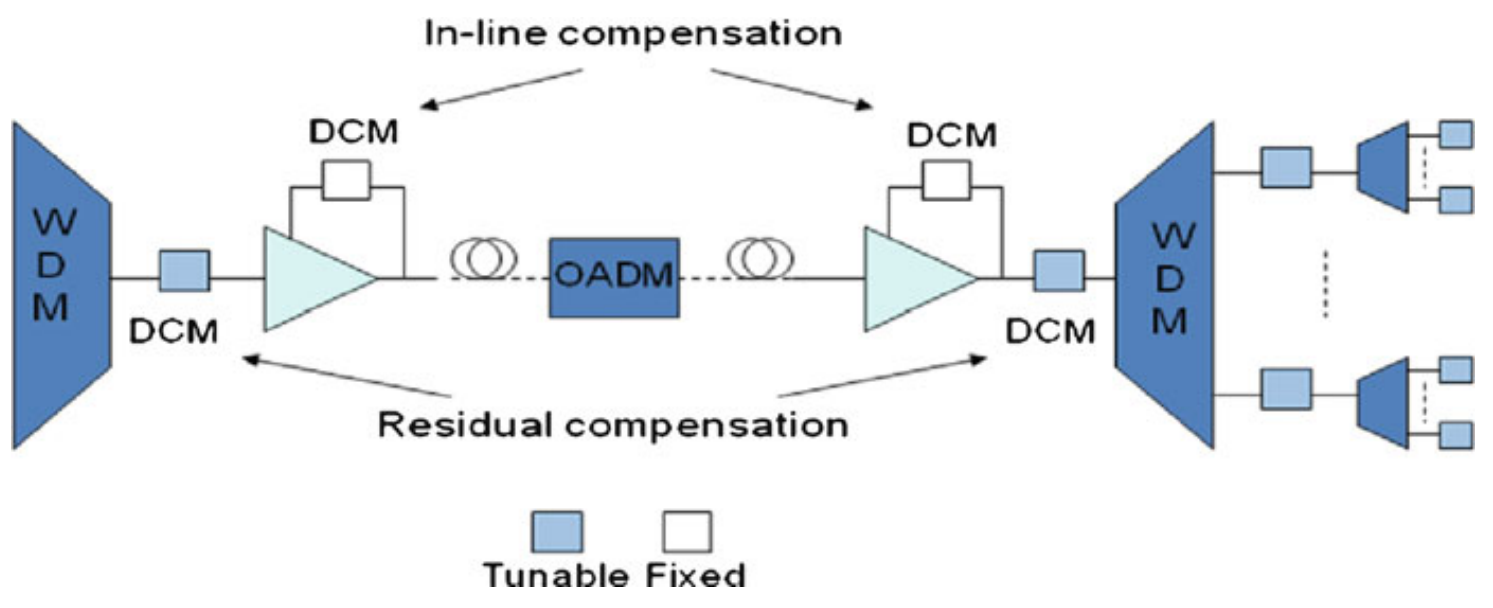

Fig. 2.27 General scenario of a WDM transmission link with different dispersion compensation modules (DCM). OADM, optical add-drop multiplexer

around 1,000 km without need for optical regeneration of the digital signals. In this scenario, it is absolutely mandatory to keep the effects of fibre dispersion under control to guarantee the required quality of transmission.

A general scenario of a DWDM amplified fibre link with chromatic dispersion compensation is shown in Fig. 2.27.

In order to exploit the existing SSMF links under the emerging transmission standards, the CD effect is one of the most important issues to take into account. Signal regeneration and $\mathrm{CD}$ compensation in the optical domain are two approaches to solve the problem. Monitoring techniques to estimate the accumulated $\mathrm{CD}$ in reconfigurable and scalable optical networks are also very desirable.

Cost-effective $\mathrm{CD}$ monitoring techniques have been developed in order to manage reconfigurable and scalable optical networks, in which the accumulated CD may change. Online CD monitoring with no need for tunable filters has been demonstrated by adding small sinusoidal components (pilot tones) to the WDM optical signal, either employing amplitude modulation (AM) (Petersen et al. 2002) or phase modulation (PM) (Park et al. 2003).

Figure 2.28 illustrates the RF-fading effect of an AM pilot tone, showing the baseband magnitude response of an SMF link with a length of $10 \sim \mathrm{km}$. The figure has been obtained using the software VPItransmissionMaker ${ }^{\mathrm{TM}}$ Cable ..... v.7.0.1.

The magnitude of the received AM pilot tone at a fixed frequency near those resonance values also change with the accumulated $C D$ of the fibre link, so the measurement of this magnitude can be employed as the basis of a $\mathrm{CD}$ monitoring technique (Petersen et al. 2002).

A different approach for avoiding the effects of signal degradation after transmission is optical regeneration, which consists of three steps (3R) of signal processing,: firstly, an optical amplification and amplitude equalisation of the signal $(1 \mathrm{R})$; secondly, a reshaping of the previously amplified signal (2R); and finally, a transmission of the reshaped signal following a retiming obtained with a clock 


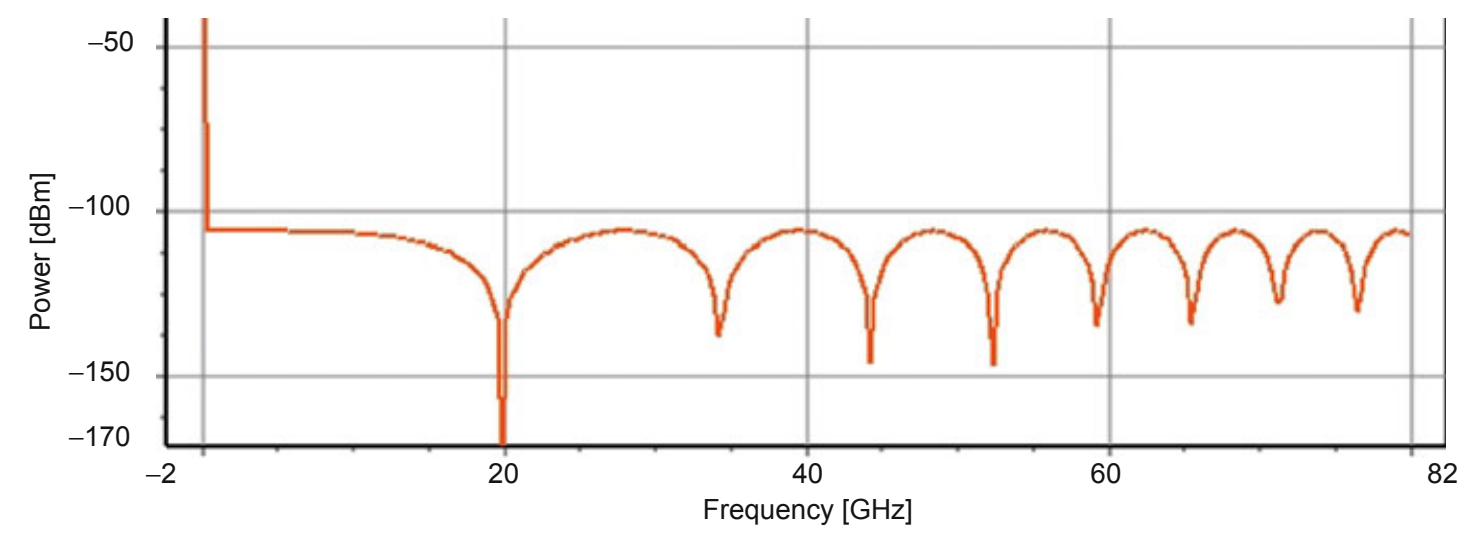

Fig. 2.28 Magnitude response of an AM fibre-optic link with a length of $10 \mathrm{~km}$ and ideal components except for fibre dispersion $(D=16 \mathrm{ps} / \mathrm{nm} \cdot \mathrm{km})$ and attenuation $(A=0.2 \mathrm{~dB} / \mathrm{km})$ at 193.1 THz

recovery stage (3R). Simple amplification and reshaping of the signal (2R) are usually sufficient to avoid problems derived from the amplitude noise and extinction ratio degradation. Nevertheless, random time deviations of optical pulses can, in some cases, arise from the interaction between ASE and signal, caused by fibre dispersion. In this case, a synchronous pulse stream clock recovery is required to keep timing jitter below acceptable levels.

Other approach to manage signal degradation in high capacity long-haul transmission systems is the dispersion compensation approach. Equalised optical amplification and filtering to reduce noise can manage, in some cases, the power attenuation in optical fibre, but chromatic dispersion must also be taken into account. The effect of $\mathrm{CD}$ can be addressed either by DCF with negative dispersion coefficient, using advanced fibre Bragg gratings or employing optical filters.

In the following, we will focus on the optical filter approaches for dispersion compensation.

All-pass optical filters are excellent candidates to perform dispersion manage-ment in long-haul WDM networks because they can be designed following a desired periodical phase response without inducing any amplitude distortion and with low insertion losses. With the current integrated-optics fabrication technologies, compact and lightweight devices can be obtained for all-optical CD managing.

Since more than two decades, fibre-optic interferometers such as the FP and the RR have been taken into account as possible laser chirp and chromatic dispersioncompensating filters in high-bit-rate digital transmission systems (Gnauck et al. 1990) (Dilwali 1992) PLC in silica waveguides were demonstrated shortly afterwards as optical dispersion equalisers (Takiguchi et al. 1994) by synthesising a FIR lattice filter with five MZI.

Novel techniques based on RR lattice architectures were proposed as general optical filter design and synthesis algorithms (Orta et al. 1995; Madsen 1996), and chips designed with these methods were fabricated using Ge-doped silica planar waveguides on silicon substrate (Madsen 1996). Following the general design and 
Fig. 2.29 (a) Single-stage optical all-pass filter based on the RR and (b) multistage all-pass filter architecture using RRs in cascade (Madsen 1998; Vargas et al. 2010) a

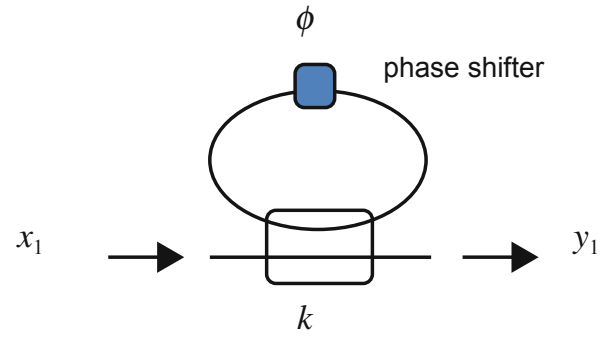

b

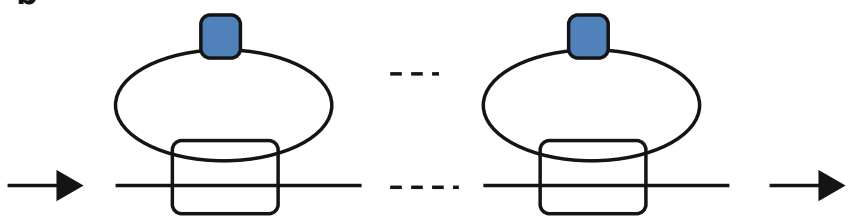

synthesis framework of all-pass filters proposed for dispersion control applications in (Madsen 1998; Lenz 1999), a two RR in series filter (Madsen et al. 1999) and a four stages Fabry-Perot tunable micro-electro-mechanical (MEM) actuated all-pass filter (Madsen et al. 2000) were fabricated (Fig. 2.29).

The ring resonator with lossless coupler and lossless feedback path is a singlestage all-pass filter (Madsen 1998). In Fig. 2.30, the group delay and the quadratic dispersion of a 6th order RR-based all-pass filter with spectral periodicity of 25 $\mathrm{GHz}$ is simulated versus optical frequency using VPIphotonics ${ }^{\mathrm{TM}}$ software, using ideal couplers and phase-shifters inside the RR with $0.1 \mathrm{~dB}$ of loop attenuation. It can be seen how the left sideband shows a negative quadratic dispersion around $-2,400 \mathrm{ps} / \mathrm{nm}$, which is enough, in principle, for compensating the chromatic dispersion of a fibre span of standard SMF with a length of $150 \mathrm{~km}$.

The analysis of a Sagnac (SG) loop in RR has been reported as a tunable optical filter (Vargas et al. 2001; Vargas 2007) with ultra-narrow bandwidth for use in Dense WDM systems; see Fig. 2.31.

This configuration is a second-order all-pass filter in a single stage and can be cascaded, as in the case of the single ring resonator in order to form a multistage photonic architecture for digital IIR filter synthesis. The configuration offers the advantages of avoiding the use of phase-shifters and a simple mechanism through the coupling factor $\kappa_{2}$ for fixing the frequency of the zeros and poles, which appear as two complex conjugated pairs. On the other hand, it also offers a considerable immunity to variations in the ring length due to the fact that the clockwise and counterclockwise recirculations propagate along the same optical length. The magnitude distortion induced by a single stage is only caused by the excess loss of the optical coupler, as in the case of the RR all-pass filter, so it is not a critical restriction.

A modified RR-SG configuration with different transmission functions in the clockwise and counterclockwise recirculations has been proposed as novel all-pass filter photonic structure and studied following the Z-transform formalism in (Montalvo et al. 2008). Under certain conditions, it is demonstrated that a modified 


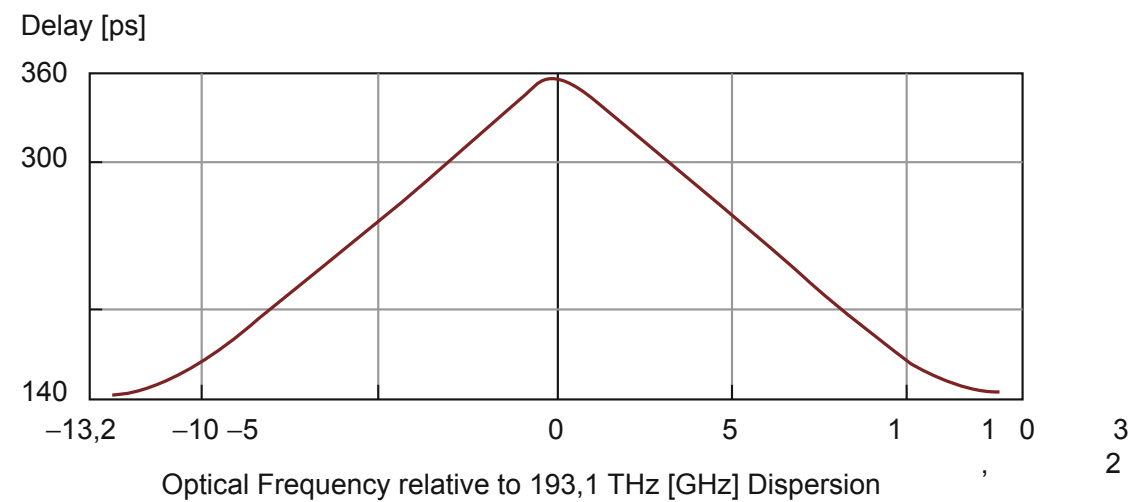

$[1 \mathrm{e} 3 \mathrm{ps} / \mathrm{nm}]$

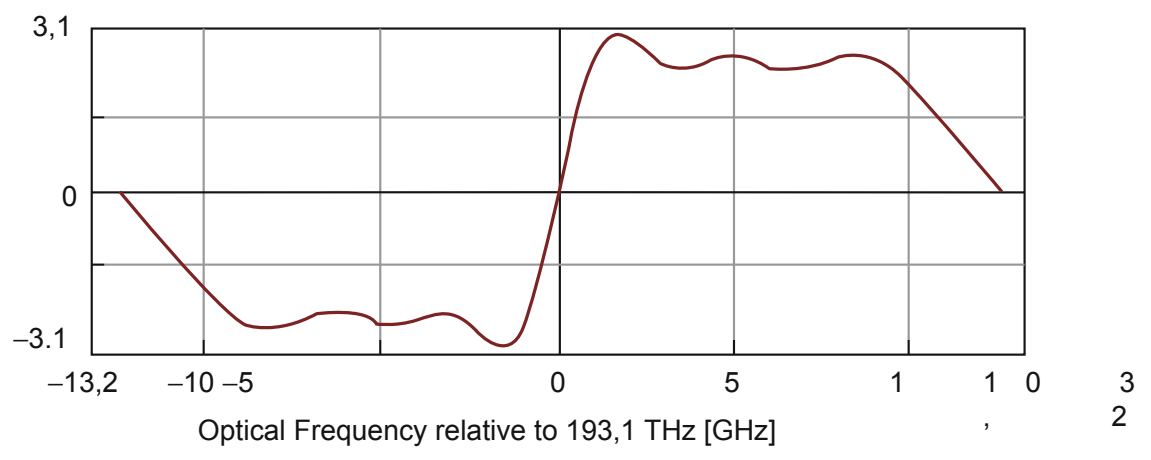

Fig. 2.30 Group delay and quadratic dispersion of a 6th-order RR in series design for chromatic dispersion compensation

Fig. 2.31 General filter architecture consisting of a ring resonator with internal transmission transfer functions $(T x, T y)$ and a Sagnac loop as transmission-reflection function (TRF). LT is the total length of the feedback path

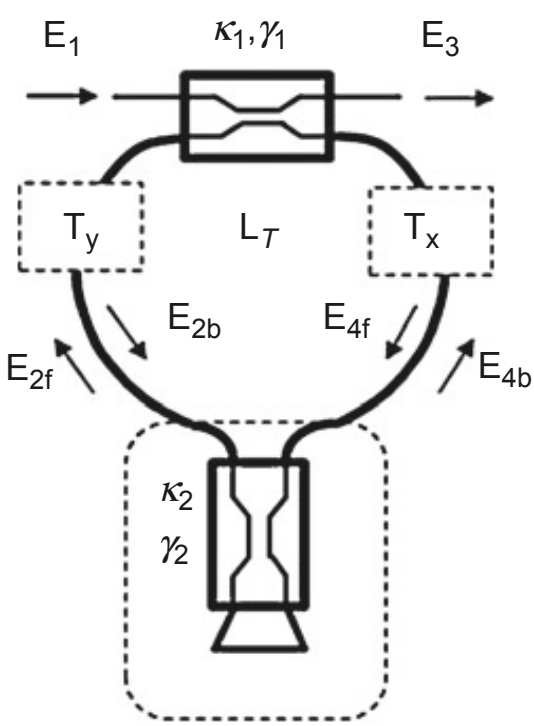

Sagnac loop

RR-SG configuration using integrated optical circulators and amplifiers can perform as an all-pass filter response with no magnitude distortion and arbitrarily high quadratic dispersion peaks. 
Simulations of optical components and networks using PDA software are the next step after the design and photonic synthesis procedures of an optical filter, being very important before the fabrication of an integrated chip, which can be extremely expensive.

Simulations are very important to study the effect of employing an optical filter in an optical transmission link for in-line chromatic dispersion compensation because the impact of the filter in the link cannot be completely derived by the shapes of its quadratic dispersion and its magnitude response but rather depends on several other aspects, such as the bit rate and the optical signal to noise ratio at the receiver's end.

On the other hand, the value of the quadratic dispersion can be related, in principle, with a specific length of a fibre link. Quick and flexible simulations are a cost-effective and useful tool to consider all these aspects, thus providing information related to the performance of an optical filter which is not evident from the observation of its transfer function or its quadratic dispersion.

\subsubsection{FBG Telecommunication Applications in Compensation and Signal Processing}

During the past couple of years, FBGs-based dispersion compensators have become a real alternative to the incumbent DCFs. This results from the fact that the FBG solution brings several advantages. Its low insertion loss provides significant cost saving and OSNR improvement through the decrease in amplification requirements. For instance, when used in-line, FBG-based dispersion compensators associated to single-stage EDFAs can replace DCF-based compensators in dual-stage EDFAs, providing a more cost-effective solution. Moreover, FBGs are compact devices that are easy to manufacture and that provide negligible distortion.

In parallel to this important development, FBGs have also been used for optical pulse shaping and manipulation purposes dedicated to ultrafast optics. They have indeed the potential to replace the bulk or micro-optics configurations that are bulky, costly and that cannot be easily integrated in fibre optics.

This section focuses on the major achievements obtained by the use of linearly CFBGs in the fields of chromatic dispersion compensation, optical pulse shaping and tunable delay line.

An FBG consists in a permanent and periodic (or quasi-periodic) modulation of the core refractive index over a certain fibre length. This modulation is often created by exposure to an ultraviolet interference light pattern incident transversely along the fibre. The periodic nature of the index modulation yields a resonant spectral response. Indeed, the FBG reflects light preferentially at the Bragg wavelength defined by $\lambda B=2 \cdot n_{\mathrm{eff}} \Lambda$ where $n_{\mathrm{eff}}$ is the average refractive index of the fibre core and $\Lambda$ is the grating period (Othonos 1999).

Contrary to uniform FBGs, the period of chirped FBGs varies along the fibre axis at a rate (also called chirp coefficient) that can reach several $\mathrm{nm} / \mathrm{cm}$. A location $\mathrm{z}$ along a CFBG reflects light at the wavelength $\lambda(z)=2 \cdot n_{\mathrm{eff}} \Lambda(z)$ where $\Lambda(z)$ stands for the local index modulation period at the position $z$ (Othonos 1999). 

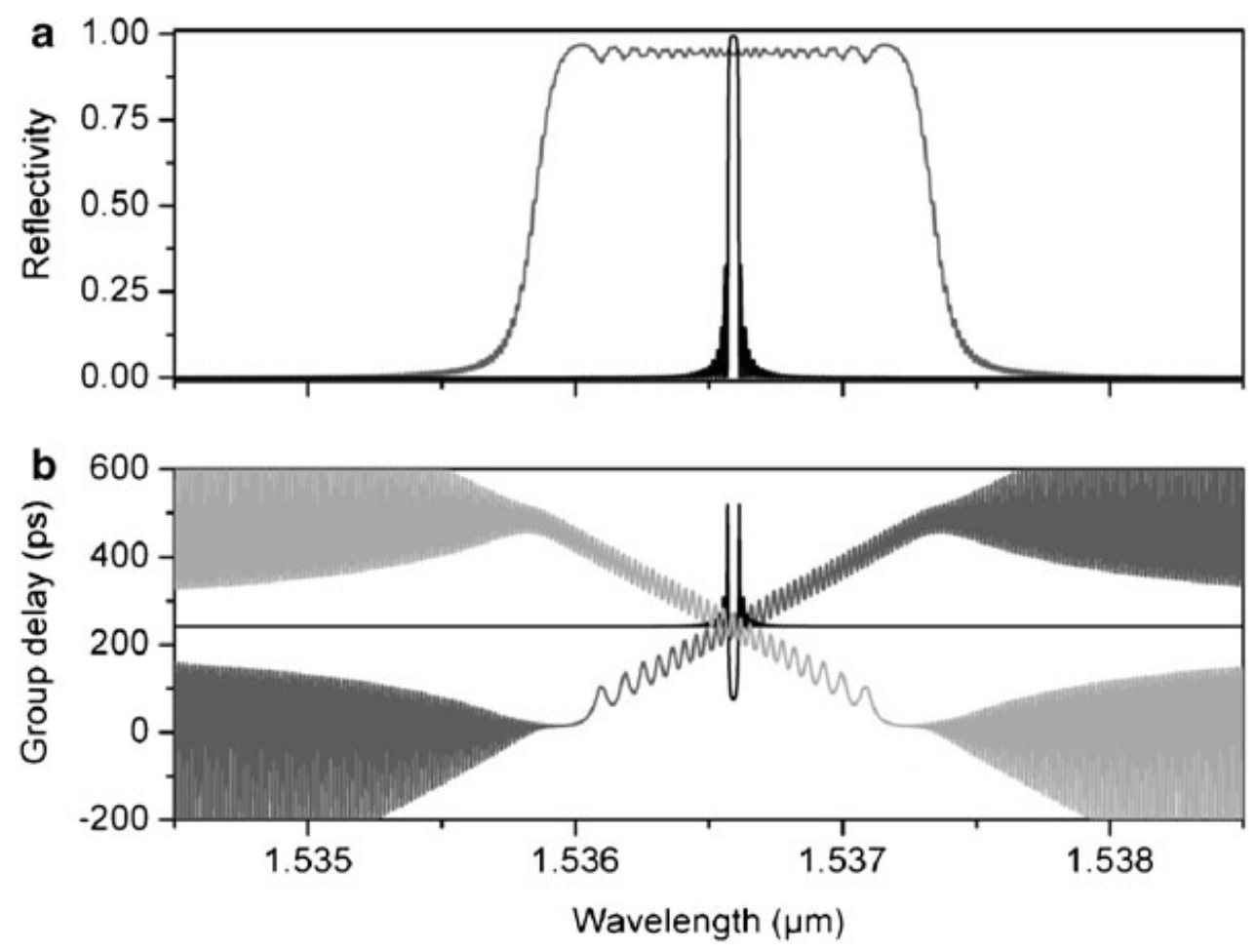

Fig. 2.32 (a) Typical reflected spectra and (b) associated group delay evolutions for uniform (black curve) and chirped (grey curves - light launched through the short-wavelengths port (dark grey), light launched through the long-wavelengths port (light grey)) FBGs (parameters used for the simulation: grating length $L=5 \mathrm{~cm}$, nominal grating periodt $\Lambda_{4} 0=530.0 \mathrm{~nm}$, chirp coefficient $C=0.1 \mathrm{~nm} / \mathrm{cm}$ and refractive index modulation $\delta n=2.510){ }^{4}$

Figure 2.32 a presents the typical reflected amplitude spectra of both a uniform and linearly chirped FBGs obtained by means of the coupled mode equations. A total chirp of $0.5 \mathrm{~nm}$ is defined along the grating length, yielding a CFBG reflection bandwidth (grey line) of about $1.50 \mathrm{~nm}$, nearly one order of magnitude higher than the one of the uniform FBG $(0.20 \mathrm{~nm}$, dark line). Figure $2.32 \mathrm{~b}$ depicts the group delay evolutions in reflection of both gratings.

The group delay of uniform FBGs strongly evolves in narrow wavelength regions (a few picometers) matching the edges of the reflection band. The evolution with wavelength of the group delay of chirped FBGs is rather different: it monotonously evolves on the whole reflection band, starting from zero (a maximum value) at the beginning and reaching a maximum value (zero) at the end of the grating when the light is injected through the short (long) wavelengths port. The maximum value of the delay (in ps) is equal to twice the grating length divided by the light velocity in silica. The slope (dispersion in $\mathrm{ps} / \mathrm{nm}$ ) of the group delay evolution is positive (negative) when the light is injected via the short (long) wavelengths side.

The CFBG dispersion can readily reach several hundreds of $\mathrm{ps} / \mathrm{nm}$. Deviations of the group delay spectrum from a straight line are known as group delay ripple. Depending on the grating reflectivity, this ripple can reach several percents of the mean group delay value. As it can impair the system performances, the ripple should be practically as small as possible. It is the reason why apodisation profiles of the 

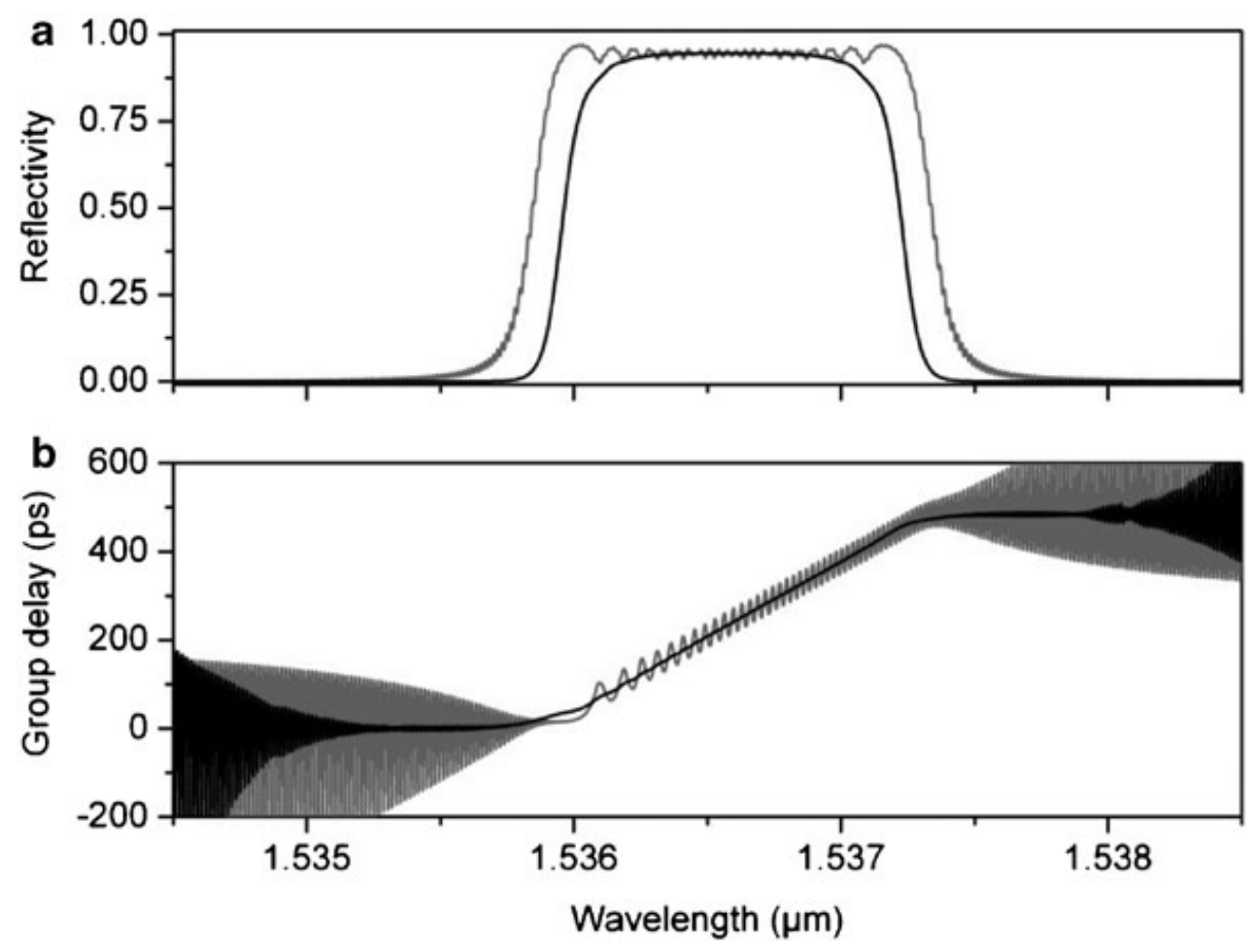

Fig. 2.33 (a) Reflected spectra and (b) associated group delay evolutions for a CFBG without apodisation profile (grey curve) and with a hyperbolic tangent apodisation profile (black curve). The parameters used for the simulations are similar to those used in Fig. 2.32

refractive index modulation are privileged for CFBGs used in telecom applications. They indeed reduce the internal interference effects, which in turn decreases the phase ripple. To illustrate this point, Fig. 2.33 shows a comparison between a CFBG without apodisation and a CFBG characterised by a hyperbolic tangent refractive index modulation profile. For the apodised grating, the reflected bandwidth is slightly reduced (a few tens of picometers), while the ripple is strongly decreased. Hence, a careful design of the CFBGs physical parameters is fundamental for a correct operation in practical applications.

CFBGs have been fabricated by using several different methods combined with the use of ultraviolet lasers emitting around $240 \mathrm{~nm}$ (continuous-wave frequencydoubled argon-ion laser or pulsed excimer laser). In practice, the chirp is often induced by varying the physical grating period along $\mathrm{z}$. In the commonly used dual-beam holographic technique, the fringe spacing of the interference pattern is made non-uniform by using dissimilar curvatures for the interfering wavefronts, resulting in variations of the period (Othonos 1999). Many other inscription techniques have been reported. For instance, CFBGs have been fabricated by tilting or stretching the fibre, by using strain or temperature gradients and by stitching together multiple uniform sections. The most straightforward inscription technique remains the use of specific phase masks that contain the desired chirp profile.

Fibre chromatic dispersion, i.e. the dependence of the refractive index value as a function of the wavelength, leads to temporal distortion of optical pulses as they 


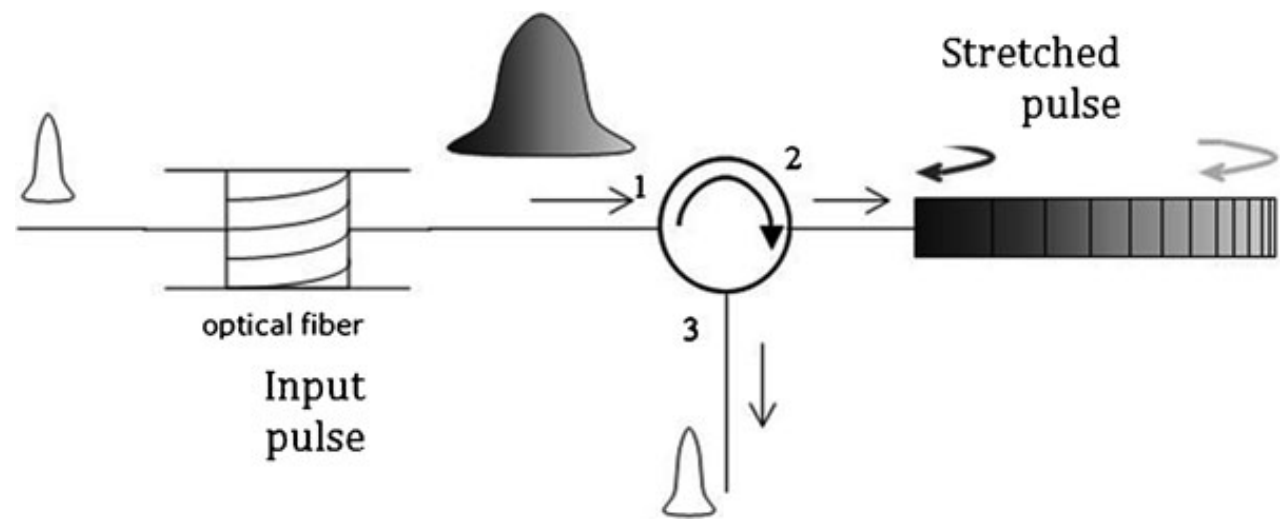

Fig. 2.34 Operating principle of a CFBG pulse stretcher

traverse an optical fibre. As a consequence, this distortion of the signal leads to inter-symbol interferences and consequently, must be compensated. One traditional mean used to overcome the issue of dispersion is to incorporate bundles of DCFs throughout the network. This is a quite straightforward technique that is based on optical fibres having a dispersion coefficient of opposite sign in comparison to standard single-mode fibres used in the network. DCFs have a typical dispersion coefficient four to eight times that of standard single-mode fibres. This level of dispersion is achieved at the expense of a fibre core diameter reduction, which in turn increases the optical loss and limits the levels of optical power that can be sustained without inducing non-linear effects.

Fibre chromatic dispersion compensation using highly reflective CFBGs is based on the introduction of wavelength-specific time delays through the use of precisely designed CFBGs (Ouelette 1987). By combining such a CFBG with a three-port optical circulator, a compact and effective dispersion compensation module can be readily realised. Figure 2.34 illustrates pulse compression by a CFBG with a period that decreases away from the entrance point. Longer wavelengths (dark grey) are reflected early along the grating, while shorter wavelengths (light grey) are reflected later near the back. The optical pulses launched into the CFBG are dispersed temporally after propagation in a bundle of optical fibre. The CFBG placed in the set-up is designed to recompress them to their original shape.

In practice, more elaborate period profiles can be realised to generate complex spectral dispersions. Moreover, the modulation amplitude of the CFBG can be tailored longitudinally in order to shape spectrally the grating reflectance.

The potential of CFBGs for dispersion compensation was demonstrated during the $1990 \mathrm{~s}$ in several transmission experiments. In 1995, chromatic dispersion compensation over $160 \mathrm{~km}$ of standard single-mode fibre at 10 and $20 \mathrm{Gbit} / \mathrm{s}$ was realised (Kashyap et al. 1995). One year later, a 12-cm-long CFBG was used to compensate the dispersion accumulated over $270 \mathrm{~km}$ of fibre at $10 \mathrm{Gbit} / \mathrm{s}$ (Laming et al. 1996). Since then, the transmission distances have been increased up to a few hundreds of kilometres using a few centimetres long apodised CFBGs, which is really impressive with so compact devices. 


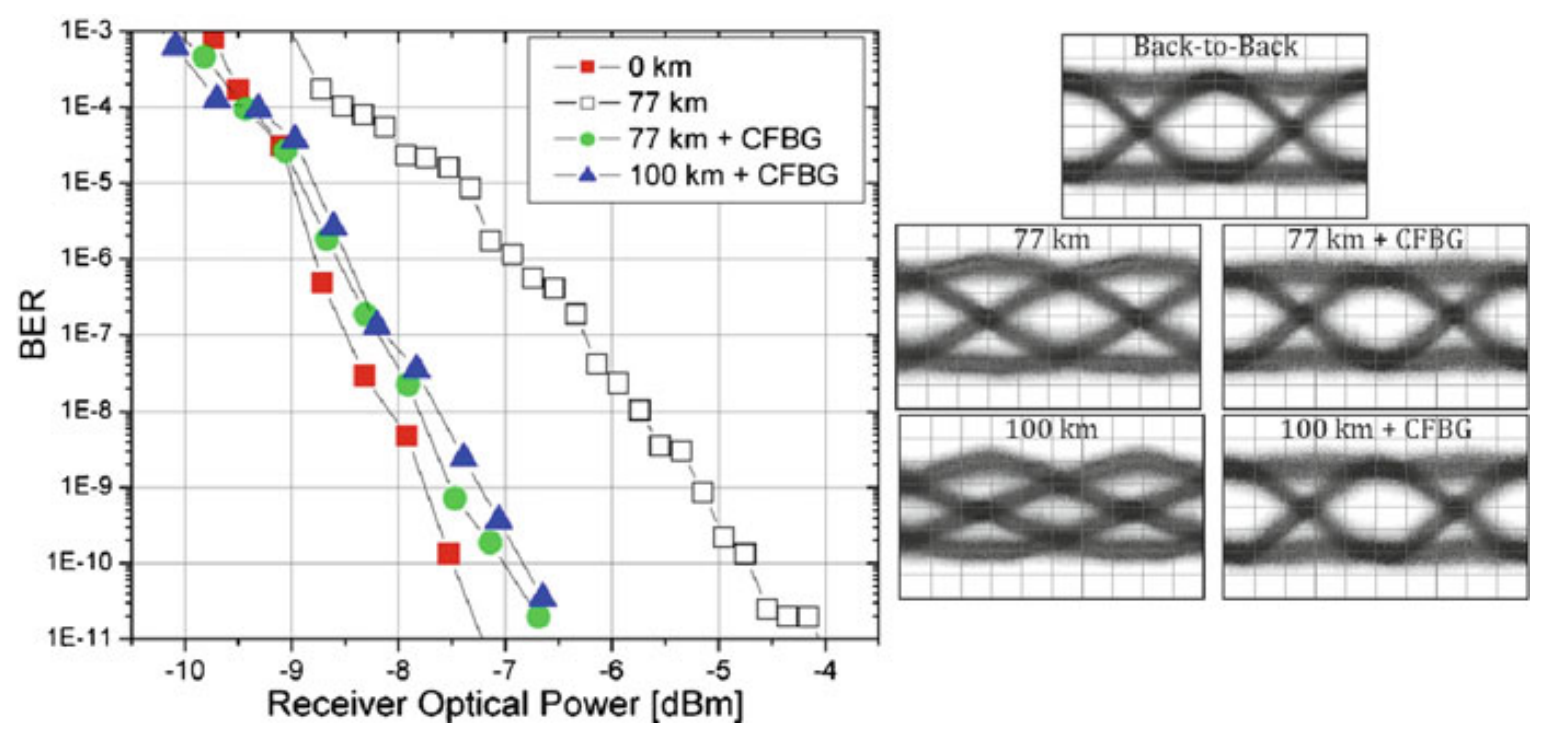

Fig. 2.35 BER measurement as a function of the received optical power and corresponding eye diagrams (scale: time $20 \mathrm{ps} / \mathrm{div}$ and amplitude $25 \mathrm{mV} / \mathrm{div}$ - power level at the receiver: $-5 \mathrm{dBm}$ ) illustrating the dispersion compensation by a CFBG

To illustrate this behaviour, measurements were done at $10 \mathrm{Gbit} / \mathrm{s}$ using an $\sim 8-\mathrm{cm}$ long linearly chirped FBG characterised by a chromatic dispersion of about $1,450 \mathrm{ps} /$ $\mathrm{nm}$. An optical attenuator was placed in the set-up to record the performances as a function of the received optical power. Figure 2.35 presents the evolution of the BER for different tested configurations ( 77 and $100 \mathrm{~km}$ of optical fibre). One can see the effect of the CFBG dispersion compensation that decreases the BER by several decades for a given received power. This effect can also be evaluated on the corresponding eye diagram measurements. Due to the chromatic dispersion induced by the bundle of optical fibre, the eye aperture strongly decreases. With the CFBG compensator, the eye retrieves its original shape, as in the back-to-back measurement.

Two main types of FBG-based dispersion compensators are commercially available nowadays: multichannel (or channelised) and broadband. The channelised version provides specific compensation for the different wavelength channels used in an optical network. The broadband type provides, in much of the same manner as a DCF, continuous compensation through the $\mathrm{C}$ or $\mathrm{L}$ band. In addition, the dynamic compensation of the chromatic dispersion has also been demonstrated through thermal or mechanical actuation of CFBGs.

As CFBGs are dispersive elements in which the group delay is a function of wavelength, they can also be used to manipulate the amplitude and phase of light and consequently, to shape pulses as the amplitude interacts with the reflectivity and the phase with the dispersion. In the Born approximation regime, this interaction maybe separated by manipulating the amplitude separately from the phase. Pulse shaping by CFBGs was first investigated by Rotwitt et al. in 1994 (Rotwitt et al. 1991). Since then, a number of different schemes have been reported, including the use of cascaded gratings with different dispersion characteristics. In such a scheme, a first CFBG is used as a pulse shaper, while the second acts as a dispersion compensator, 


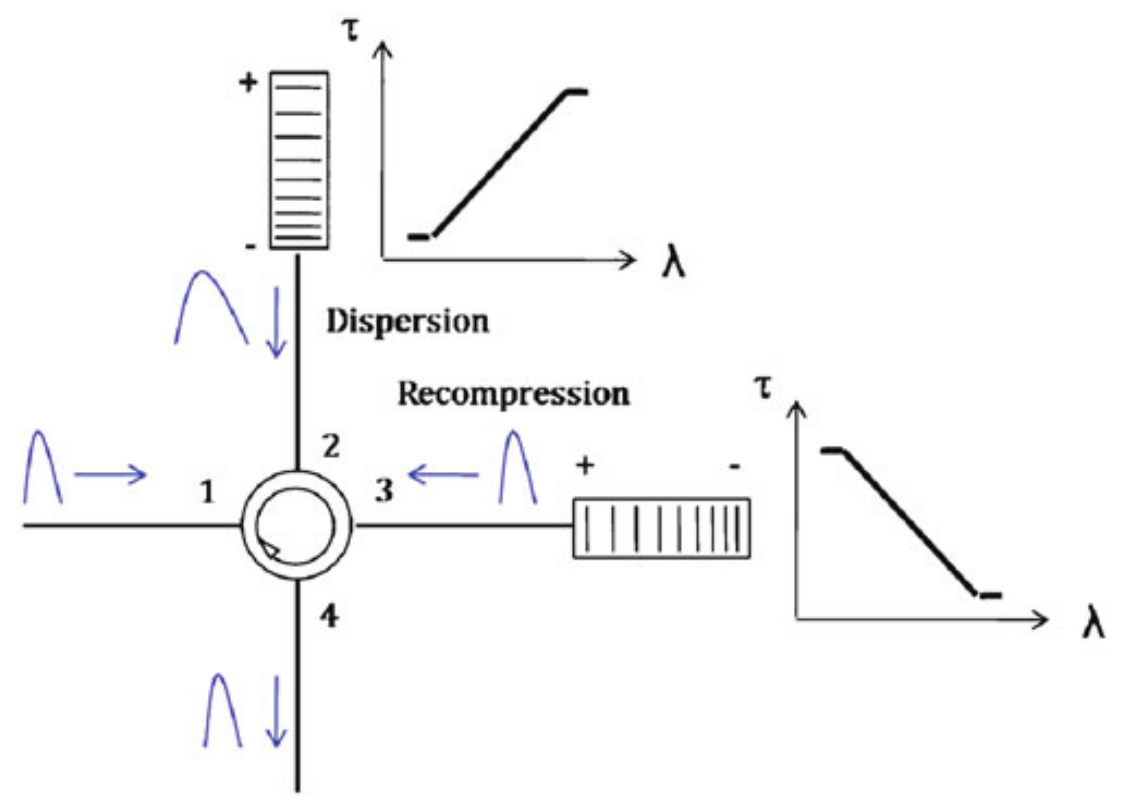

Fig. 2.36 Schematic of a twin CFBG delay line

in a similar way as the scheme presented in Fig. 2.34. With this principle, it is possible to generate triangular or square pulses. CFBGs can also be used to multiply the repetition rate of a stream of pulses, via the application of the temporal Talbot effect.

CFBGs have also been widely used for the realisation of tunable optical delay lines. When a CFBG is stretched, the point at which the incoming pulses are reflected changes while the pulses are simultaneously dispersed (Choi et al. 2005). This dispersion can be practically cancelled by using a second identical grating placed in the opposite direction. In other words, the pulse is recompressed providing that two gratings are used in succession with the sign of the chirp reversed for the second grating, as depicted in Fig. 2.36. By stretching one of the gratings, the physical delay between them is altered, and a time delay is introduced. In practice, the time delay is generally tuned by shifting the CFBG resonance band through thermal or mechanical actuation. Because wavelength shifts are induced, optical pulses with a linewidth comparable to that of the CFBG reflection bandwidth cannot be variously delayed without undergoing important distortions. Moreover, there is an increasing mismatch between the overlap of the reflection spectra of the two gratings with increasing strain. This can be detrimental, especially in the framework of high-speed transmissions with bit rates higher than a few tens of Gbit/s.

To alleviate these drawbacks, a novel set-up has been proposed to generate a tunable delay with a single CFBG (Caucheteur et al. 2010). Instead of using directly the CFBG group delay curve, this solution exploits the DGD evolution. In practice, orthogonally polarised pulses are sent through both ports of the CFBG while its local birefringence is controlled. This leads to a dynamic evolution of the DGD inside the CFBG reflected bandwidth so that tunable delays can be achieved. Two significant advantages are therefore obtained. This set-up does not require any wavelength shift. 


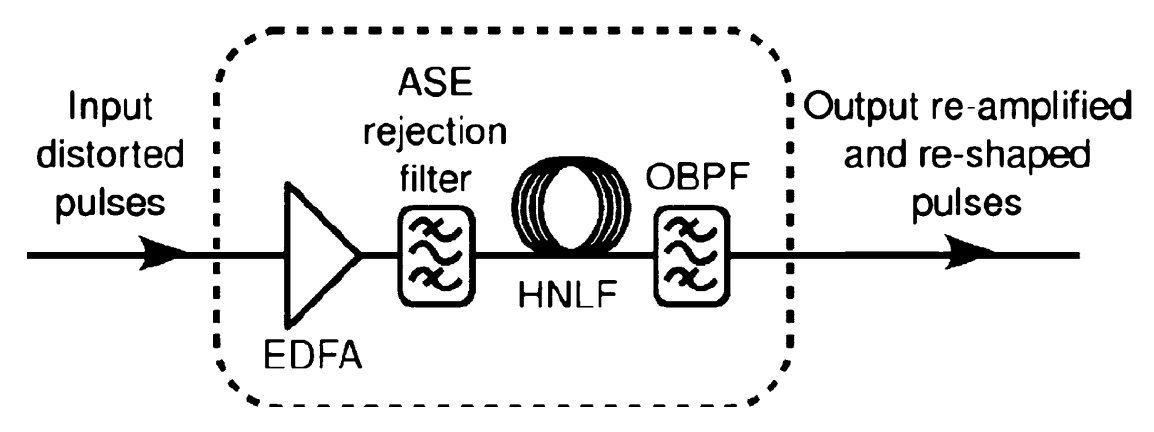

Fig. 2.37 Schematic of the single-cell SBF regenerator. $E D F A$ erbium-doped fibre amplifier; $A S E$ amplified spontaneous emission; $H N L F$ highly non-linear fibre; $O B P F$ optical band-pass filter

Consequently, the full CFBG bandwidth can be used to delay optical pulses nearly as broad as the grating reflection band. The double pass of light inside the same CFBG (in both directions) allows to compensate the chromatic dispersion, without the need for a second CFBG perfectly matched with the first one.

In conclusion, CFBGs present spectral characteristics (amplitude and phase) well suited for nowadays optical telecommunication applications, not only for chromatic dispersion compensation but also for pulse shaping and the realisation of tunable optical delay lines.

\subsubsection{All-Optical Regeneration/Buffer}

It is an important requirement for an optical network, comprised of multiple pointto-point links, that the signals propagating throughout be of sufficient quality to detect. Historically, this has been achieved by the use of electrical repeaters. These convert the incoming optical signal into an electrical signal from which the base data is recovered before being used to transmit a new optical signal. This OEO conversion is undesirable when striving for high-bit-rate systems in which the conversion becomes a limiting factor.

Optical amplifiers have removed the OEO conversion but added ASE noise to the optical signal. An optical regenerator adds additional processing functions, such as amplitude equalisation (reshaping) and temporal repositioning (retiming) of the optical pulses.

The purpose of an optical regenerator is to process input distorted pulses, minimising signal degradation through the impact of noise, and contribute an output in which a receiver is better able to extract the original data.

Although there are a selection of optical regeneration schemes, one particularly interesting version, proposed by Mamyshev et al. (1998) is based on the principle of self-phase-modulation-induced spectral broadening followed by offset filtering, which will henceforth be referred to as a SBF regenerator.

Figure 2.37 shows a single SBF cell which consists of a high-power optical amplifier (EDFA), a HNLF and an OBPF. It is useful to include an additional filter after the EDFA to suppress out-of-band ASE noise. 

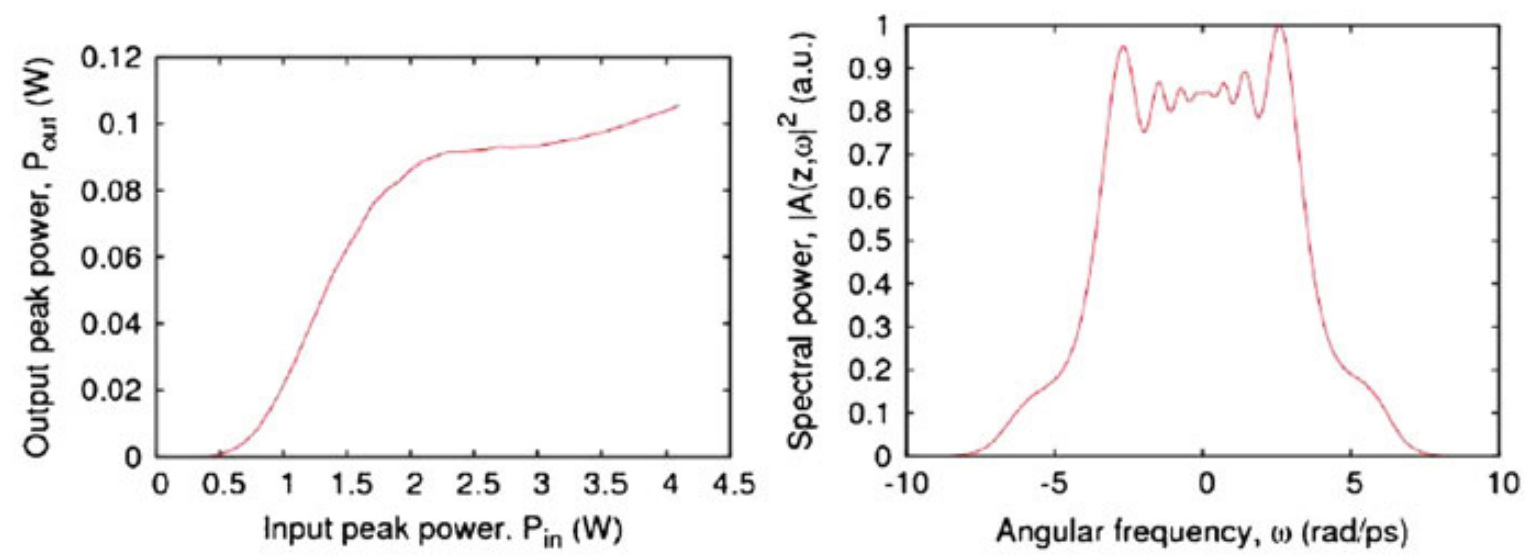

Fig. 2.38 Power transfer function for single pulse passing through one SBF cell (left). Spectral plot at the output of HNLF for an input peak power of $2.6 \mathrm{~W}$ (right); spectral power is normalised (arbitrary units)

The EDFA amplifies the peak power of input pulses to a level where considerable spectral broadening can take place within the HNLF due to self-phase modulation. After propagating through the fibre, the spectrum is sliced by the OBPF at a frequency offset to that of the input centre frequency of the pulses.

It is possible to add a second SBF cell after the OBPF of the first cell, allowing the recovery of the original centre frequency of the pulses. This is achieved by using an equal and opposite offset frequency for the OBPF in the second cell.

A plot of PTF for a regenerator shows the peak power exiting the SBF cell (after the offset filter) as a function of the peak power entering the SBF cell (before the amplifier). The input data to the regenerator are represented by RZ pulses, for which zero bits correspond to pulses with (ideally) no power and one bit correspond to pulses with power greater than a fixed threshold level. An ideal PTF for a regenerator is a step function, in which the output peak power is zero for input pulses with peak power below the threshold level and a constant output peak power for input pulses above the threshold.

Generation of the PTF is the first step for regenerator parameter optimisation. The operating region is located at the plateau after the first maxima of the function, and it is here that amplitude jitter (the variation in peak power from that of the average for the pulses) may be reduced. Amplifier gain is adjusted so that input pulses have the required peak power to enter this region. The PTF also provides information on the suppression of ghost pulses, which are low-power pulses located within a "zero" bit.

Figure 2.38 shows a typical PTF in which input pulses with peak power less than $0.5 \mathrm{~W}$ are output with almost zero output power, while input pulses with peak power in the range 2.2 to $3.3 \mathrm{~W}$ are output at the almost constant peak power of $0.09 \mathrm{~W}$. The SBF regenerator parameters are given in Table 2.2 for a carrier wavelength of $1.550 \mathrm{~nm}$.

Figure 2.38 also shows a spectral plot at the exit of the HNLF for a peak input power of $2.6 \mathrm{~W}$. 
Table 2.2 SBF

regenerator parameters

\begin{tabular}{ll}
\hline Attenuation coefficient, $\alpha$ & $2.13 \mathrm{~dB} / \mathrm{km}$ \\
\hline Dispersion, $D$ & $-1.7 \mathrm{ps} /(\mathrm{nm} \cdot \mathrm{km})$ \\
Dispersion slope, $S$ & $0.023 \mathrm{ps} /(\mathrm{nm} 2 \cdot \mathrm{km})$ \\
Fibre length, $L$ & $1 \mathrm{~km}$ \\
Non-linear parameter, $\gamma$ & $18(\mathrm{~W} \cdot \mathrm{km})-1$ \\
Filter offset & $375 \mathrm{GHz}$ \\
Filter bandwidth & $71.25 \mathrm{GHz}$ \\
\hline
\end{tabular}

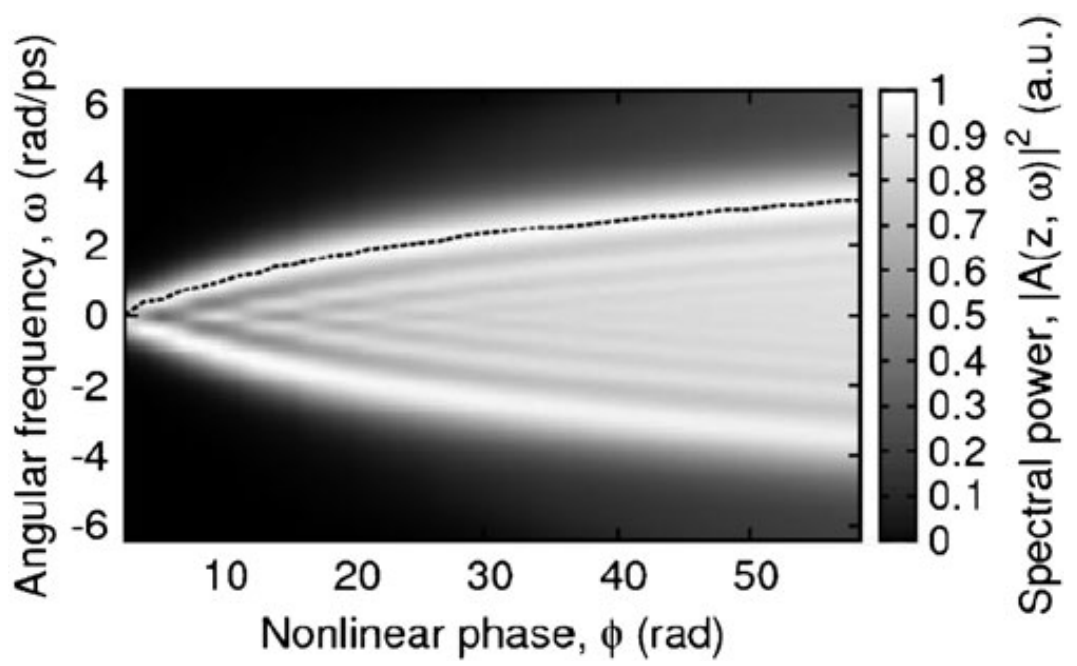

Fig. 2.39 Spectral power map showing broadening of pulse with increasing non-linear phase. Dotted line marks the position of the boundary spectral maximum

It is useful to view the spectral evolution of the input pulses as they propagate through the HNLF. As the pulse propagates through the HNLF, it acquires a nonlinear phase shift and ultimately starts to broaden. The broadened spectra will consist of multiple peaks, though it is the rightmost or leftmost peak which is of interest. Locating these spectral peaks is useful since the results of (Striegler 2006) indicate that these provide the optimised frequency for the offset filter, while the filter bandwidth should be adjusted to the spectral width of the peak.

Spectral broadening is symmetrical when there is no dispersion or second-order dispersion, while third- or higher-order dispersion causes the spectrum to become asymmetrical. When such asymmetry occurs, the higher-frequency spectral peak should be tracked in preference to the lower-frequency peak. Figure 2.39 shows an example of spectral broadening when 2nd- and 3rd-order dispersion is included with high non-linearity. The spectral peak is traced by the dotted line and indicates the ideal filter frequency in terms of non-linear phase.

Design scaling rules have been proposed (Provost et al. 2007a; Provost et al. 2007b) in which the regenerator is classified according to the shape of its PTF, then optimised according to soliton number. An optimal fibre length and filter offset frequency to bandwidth ratio may then be calculated. 
An alternative design rule considers the ratio of dispersion to non-linearity (Baveja et al. 2009). This is useful in categorising a range of SBF regenerators since those that share the same ratio are likely to display the same regeneration capabilities.

When considering noise introduced by amplifiers in an optical link, it has been shown that the inclusion of the ASE-rejection filter within the SBF regenerator is vital (Nguyen et al. 2006), with no improvement in Q-factor unless a filter is included after the amplifier. The filter serves to reduce the noise power over the pass-band of the offset filter, leading to an increase in the optical signal to noise ratio.

In the general case, where higher-order dispersion and non-linear terms are included, there is no analytical solution to the NLSE. Instead, the solution is approximated using numerical techniques.

A common strategy is to use the symmetrised split-step Fourier method (Agrawal 2007). This proceeds by splitting the NLSE into linear and non-linear terms, then applying them over a short segment of the total fibre length in each step. Initially, the field is propagated half a step using linear terms only, followed by including the non-linear terms at the midpoint of the segment, then completing the step using the linear terms over the remaining half step.

Numerical integration is required for an accurate approximation of the fibre segment non-linearity to be applied at the segment midpoint. A popular choice is the (globally) 4th-order Runge-Kutta method.

The NLSE is generally stiff, which can lead to the numerical calculation diverg-ing rapidly to infinity, even when a high precision is used during the calculation. This stiffness may be reduced by transforming to the interaction picture (Hult 2007).

Additional improvements may be included through adaptive step-size control. A particular implementation of this is step-size doubling. A fine estimate using two sub-steps of half the step size is compared to a course estimate which covers the fibre segment in one step. The absolute difference in the two estimates is compared to a user-defined tolerance. If the error estimate is less than or equal to the tolerance, then the simulation continues with the next step (and may increase the step size). Conversely, if the value is greater than the tolerance, then the current step is repeated with a reduced step size.

Alternatives to the explicit step-size doubling method include Fehlberg, CashKarp and Dormand-Prince methods. These are implicit methods in which each step is taken once only; the error estimation is calculated internally by the algorithm (normally by using a 4 th- and 5 th-order comparison).

These high-precision methods are essential when simulating pulse propagation through highly non-linear fibre. Chalcogenide glass fibres have been proposed as the HNLF within the SBF regenerator (Fu et al. 2005), which may have a nonlinear parameter of greater than $1,000(\mathrm{~W} \cdot \mathrm{km})^{-1}$, which is greater than that of standard silica fibres by more than a factor of 500 .

The SBF regenerator has received a relatively broad investigation into its range of operation and optimisation. Most of this research has been applied to a single cell version, usually in the absence of noise and attenuation. Additionally, the 
regenerator is of $2 \mathrm{R}$ classification, that of reamplifying and reshaping of input pulses. A retiming stage is therefore an interesting area of additional investigation. Since the amplifier within each SBF cell adds additional noise to the incoming bit stream, it would be useful to investigate whether such amplifiers can be removed or be reduced in number. One option is to use a fibre with such high non-linear parameter that the peak power of input pulses is adequate for regeneration without need of additional gain.

Other arrangements allow the reuse of components, typically bidirectional propa. gation in fibre (Provost et al. 2008).

\section{Impairment Control and QoT-Constrained Routing}

The trend towards service dependent QoS, the demand for guaranteed capacity to integrate telecom services in data communications, the generalisation towards more flexible meshed network topologies and the availability of highly efficient dynamic optical switching architectures bypassing digital signal regeneration leads to novel constraints on routing. The implementation of lightpath routing in a meshed optically switched WDM network is not straightforward as each new request accepted can affect the quality of other previously established circuits. To achieve a requested end-to-end QoS for a specific service, the underlying end-to-end lightpath, sequence of lightpaths in the multi-hop transmission network case, needs to offer a certain minimum quality of transmission (QoT).

The interoperability among network layers based on the introduction of autonomous control planes per layer that share information vertically must cope with the increasing complexity inherent to the deployment of reliable multilayer transport networks. The need to achieve differentiated QoS and to preserve or even enhance network reconfiguration and protection capability and autonomy is thus spreading from network layers towards the physical layer potentially comprising of a multitude of technologies (Saleh 2006).

Following a short introduction outlining the problem, we discuss schemes and approaches for QoT control related to the physical (analogue) layer zero, considering abstract optical signals and the electrical counterparts (prior $\mathrm{E} / \mathrm{O}$ and post $\mathrm{O} / \mathrm{E}$ conversion), before we return to the related impairment constraint routing problem.

\subsection{Impairment Control}

In contrast to QoS, QoT refers to the physical properties of lightpaths. QoT is a complex metric embracing the physical parameters of the different network components along a lightpath as well as the optical signal transmitted (modulation format, pulse rate, optical filtering). In addition, some parameters depend on the multiplex of signals sharing a resource and, therefore, are load-dependent. This 


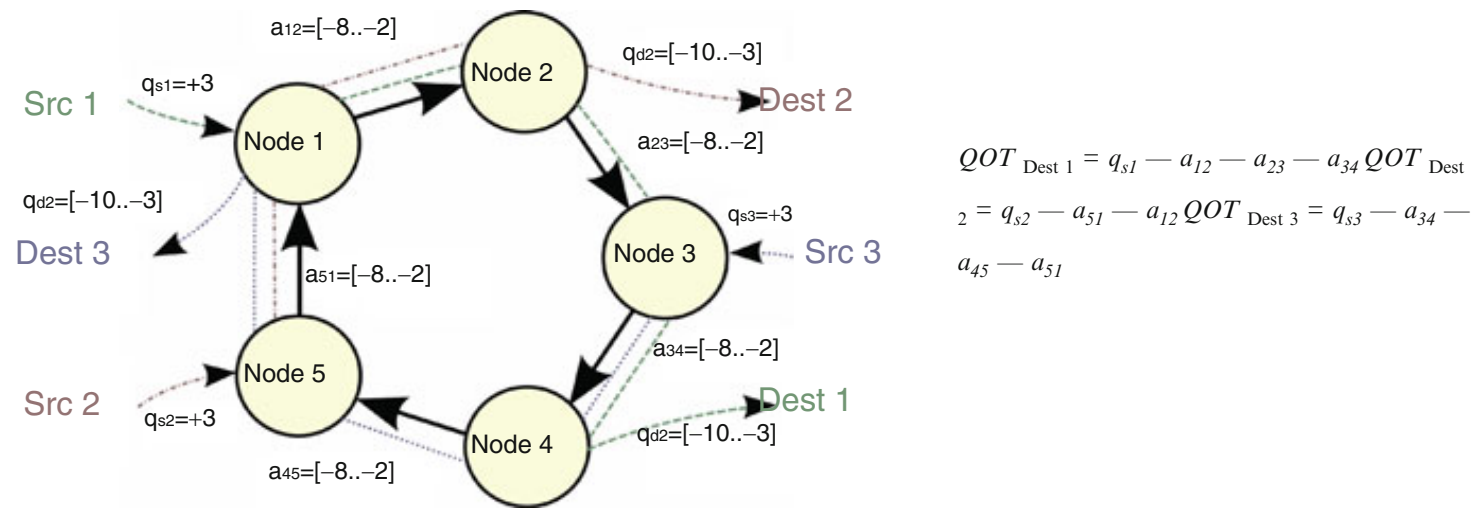

Fig. 2.40 Example illustrating interdependent adjustments

increases the difficulty in maintaining a uniform and acceptable quality for any lightpath across a transparent optical transmission network comprising a multitude of different network components and signals, being generally not feasible (Chen et al. 2004).

Proposed physical impairment compensation and mitigation possibilities yield increased optical reach; however, in contrast to regeneration of bits and bytes, it does not generally provide perfect signal regeneration. It is therefore mandatory to maintain and provide the remaining level of impairment per network element via the responsible control plane. Note that here, the term network element is used for any logically identifiable network resource from any layer, while the term network component shall be used if referring to the subset of physically identifiable network resources.

Physical impairment mitigation schemes based on processing of electrical signals after $\mathrm{O} / \mathrm{E}$ conversion (and/or before) are applicable at the ends of lightpaths only. Thus, to achieve scalable transparent transmission networks, the multi-hop transmission scenario is essential to integrate electrical impairment compensation and mitigation at intermediate nodes. Optimal (meaning cost-efficient) placement of impairment mitigation features is a hard challenge for network design. However perfectly done, it still relies on impairment-aware routing and monitoring of the remaining physical impairments to grant a QoT that supports the QoS required for offering certain transport services.

Any adjustment that influences QoT must comply with all demands raised by other lightpaths sharing a resource. For instance, optimally adjusting the gain of alloptical amplifiers being part of links carrying many lightpaths may already pose a fundamental mathematical problem. This is illustrated in Fig. 2.40 where an abstract artificial dimensionless additive QoT is used to show the mathematical problem. Demand on received QoT is -10 to -3 , degradation per link is -5 , input QoT is +3 and the compensation can be adjusted between -3 a $\mathrm{n} \mathrm{d}+3$, i.e. degradation cannot be completely compensated but deliberately worsened, as it is typical for physical impairments.

To solve the simple dimensioning problem depicted in Fig. 2.40, we have three equations for five variables, a multidimensional continuum of feasible solutions and 
thus it might be expected that an optimum solution providing equal connection quality among all three connections could be found. However, there is a cyclic dependence and solving the equation system for feasible real numbers yields no such solution. Further, as cycles like the one discussed are likely to exist in every meshed topology, we derive that, in general, solutions that grant equal quality per connection do not exist if impairments cannot be entirely removed hop-by-hop.

We must consequently drop the equal performance requirement and instead aim at obtaining stable performance with least effort, meaning minimal added equipment cost. Therefore, parameters which can be efficiently stabilised per link shall be managed by per link control loops. All other parameters should be controlled per lightpath, utilising impairment control mechanisms integrated in sender and receiver circuitry. This division between link and end-to-end functions is actually not new; it equals the link layer management and the transport layer management defined by the OSI model (ITUX200). This clearly does not grant global optimisation. Nonetheless, stable and reliable physical impairments are more important than squeezing out the least quantum of optical span, especially in the multi-hop transmission network case.

\subsubsection{Physical Impairments, QoT and Impact on QoS}

Customers demand QoS derived from SLAs, which typically consist of statistical metrics on BER, blocking/loss rate and latency among other non-QoS-related issues. Loss-rate and latency are partly related to BER, i.e. loss and repetitions of payload in case of bit errors. Response to buffer overflow and control-plane-related issues are in general not related to physical impairments. Therefore, we need to consider BER as the only QoS requirement dependent on the control of physical impairments. BER is directly related to QoT, although, the relation depends on modulation format and receiver technology. The contribution of the considered physical impairments to QoT degradation is expressed in Eq. 2.6 according to (Cugini et al. 2008).

$$
Q o T_{[\mathrm{dB}]}=Q_{\mathrm{OSNR}}-Q_{\mathrm{GVD}}-Q_{\mathrm{DGD}}-Q_{\mathrm{FWM}}-\ldots
$$

where $Q_{\mathrm{OSNR}}=P_{\text {signal }} / P_{\text {noise }}$ reflects the eye-opening at the receiver after consideration of ASE noise. The other $Q$-factors are eye-closures caused by other linear and non-linear, potentially signal or technology dependent, effects (i.e. GVD, DGD, FWM).

To establish a common parameter for control loops, it is initially assumed that the average QoT per network element is met by design. Consequently, every network element contributes a certain amount of impairments. Considering that BER is related to QoT, a virtual BER contributed per network element can be defined and, comparable to the noise factor $\mathrm{F}$ assigned to components along RF connections, used to calculate end-to-end BER. Reducing and stabilising this virtual BER per network element is the target of impairments control, and the virtual BER can be used to identify feasible lightpaths. Next, impairment compensation control loops 
Table 2.3 Assumed parameter contribution to QoT (Teixeira et al. 2009)

\begin{tabular}{llll}
\hline Parameter & Sensitivity & Impact & Speed \\
\hline$Q_{\text {OSNR }}$ & Low & Linear & Moderate \\
$Q_{\text {GVD }}$ & Low & Exponential & Slow \\
$Q_{\text {DGD }}$ & Moderate & Exponential & Fast \\
$Q_{\text {FWM }}$ & High & Non-linear & n/a \\
\hline
\end{tabular}

and their influence on actual QoT are discussed before returning to the routing problem and sketching common approaches.

\subsubsection{Impairment Control and Stabilising of Network Elements}

Traditionally, stable transmission performance is gained by controlling the environment, e.g. adding dummy channels to keep optical power constant when not all wavelengths are in use. More dynamic approaches are required since traditional ones are efficient only for entirely opaque network architectures. Reduction and stabilisation of physical parameters is the prime demand to realise reliable dynamically switched optical transmission networks. For OBS and OPS, the problem of keeping the performance of physical components constant, meaning independent of dynamically changing traffic assignments, needs to be addressed.

For stabilisation, we need to consider the dependence of the contributing components on changes of traffic load (sensitivity), their relative impact on QoT (impact) and how fast they possibly could be compensated (speed), alike the assumptions shown in Table 2.3.

Not all contributions can be considered hop-by-hop. Either can they not be monitored all-optically or there are no means to mitigate their impact all-optically. In addition, relevance of several components is dependent on the traffic load. There-fore, for dynamic environments, efficient control strategies need to be deployed.

Assuming that a virtual target BER per network element is achieved by design, the dynamic end-to-end BER is then the sum of these plus the deviations from the targets. The end-to-end deviation thus depends on the number of hops $n$ where adjustments, originated temporarily, increased BER (BER transient $)$ as follows:

$$
\begin{aligned}
B E R_{\text {transient }}\left(\text { link }_{\mathrm{i}}\right)= & p_{\text {adj }}(i) E\left[\text { biterror } \mid \mathrm{QoT}(t)<Q o T_{\text {target }}\right] \\
B E R_{\text {transient }}(\text { path })< & \sum_{i=0}^{n} B E R_{\text {transient }}\left(\operatorname{link}_{\mathrm{i}}\right)+p_{\text {adj }}(l p) \\
& \times E\left[\text { biterror } \mid Q o T(t)<Q o T_{\text {target }}\right]
\end{aligned}
$$

where $p_{\text {adj }}(i)$ is the probability to be in an adjustment state on the $i$-th link in the path, $p_{\text {adj }}(l p)$ the probability for lightpath adjustment and $\mathrm{E}\left[\right.$ biterror|QoT $\left.(t)<\mathrm{QoT}_{\text {target }}\right]$ is the likelihood of bit errors in the case that current QoT is below target QoT, meaning current BER contribution is above the planned. 
To get an upper bound, let us assume a worst case: No end-to-end head-room and complete detector malfunction for $\mathrm{QoT}(l p)<\mathrm{QoT}_{\text {target, }}$ i . e . E[biterror QoT $\left.(t)<\mathrm{QoT}_{\text {target }}\right]=1 / 2$. If we additionally simplify and assume the probability for adjustments to be equal for all links and lightpaths, Eq. 2.7 reduces to the following:

$$
B E R_{\text {transient }}<\frac{n+1}{2} p_{a d j}
$$

To minimise the transient impact, we need to minimise $p_{\text {adj }}$. A s $p_{\text {adj }}$ is calculated by occurring number of adjustments per time unit multiplied by the time units, these adjustments cause QoT $(t)<\mathrm{QoT}_{\text {target}}$; only the latter can be addressed by design of compensation mechanisms. The common problem of control loop design, precision vs. stabilisation time, arises. Along with the acknowledgement of cascaded potentially interdependent control loops, this yields the compensation performance and optimization demand.

Commonly asymptotic control loop behaviour is favoured. However, if there is headroom for sufficient detection, slight overcompensation is acceptable and thus faster operation and reduced number of biterrors per adjustment is possible. To cope with the cascades and interdependencies, a problem, as depicted in Fig. 2.40, arises. This can be handled offline using common methods since control loop performance characteristics are independent of instantaneous traffic load. Nevertheless, the results of such optimisation will depend on actual traffic statistics (distribution and characteristic) and the actual network topology that defines the cascades and interdependencies to consider.

\subsection{Current IA-RWA Approaches}

Routing, a term from graph theory, in communication networks generally performs the identification of a path (route), per connection request, between a source and a destination node across the network. In optical networks, the particular wavelength(s) along the path also need to be determined. The combined problem is in literature often called RWA problem (Zang et al. 2000). Most RWA proposals can be classified into two main categories: (a) considering the effects of physical layer impairments via iterative path testing until a feasible lightpath is found and (b) integrating physical impairments in local routing decisions. Both approaches provide IA-RWA. The latter also represents a specific variant of CBR problems with NP-hard computational complexity, in principle not scalable. However, the limited size of today's transparent networks, conditioned by the limited optical reach, alleviates this scalability issue.

Prior to discussing exemplary approaches, a principal decision is addressed: whether to implement routing as centralised or distributed. Any routing scheme can be implemented centralised, but only a few fit the demands for an efficient distributed implementation. The key demand for decentralised routing is that a 
correct decision can be made locally based on local information. If every node needs to know about everything going on in the entire network to do routing decisions, this is not economical, and a centralised approach may be considered. However, routing algorithms can be of polynomial complexity if, and only if, no prior knowledge about the reminder of a path is required for a correct routing decision. This is the case if, and only if, the Bellman (2003) or the Hamilton-Jacobi-Bellman (Bertsekas 2005) equations for dynamic programming hold.

\subsubsection{Centralised Vs. Distributed Routing}

Lightpath routing may be performed distributed, on-demand or continuously, endto-end or hop-by-hop, within the physical layer's control plane, or might be outsourced to be performed in a more centralised way by employing dedicated PCEs that provide explicit routes on request. The latter demands timely flooding of the physical parameters to all PCEs, while the first relies on sufficient information available at each node. Whichever is more efficient depends on the network size, the meshing degree and load variability. However, centralised routing may consider QoT correlation among lightpaths more easily than distributed routing. In any case, considering QoT correlation raises complexity considerably. Thus, if we may assume that the optical routing changes rarely, meaning requests for not already routed all-optical connections and therefore resource assignment changes occur from time to time only and can be scheduled, the centralised approach is, for the optical layer, expedient. This is commonly the case with multilayer networks where lower layers are optimised to statically provide maximal capacity to the layer above independent of the current capacity usage. For such situations, offline routing using ILP-based optimisation and rearranging of assignments scheduled night time is commonly applied. We need to note that (a) static virtual topologies evidently cannot utilise the full potential of statistical cross-layer multiplexing and (b) the intention to reduce the number of layers towards IP over WDM annuls the essential precondition that resource assignment changes occur only rarely.

\subsubsection{Common Sequential IA-RWA}

Most IA-RWA approaches recently proposed consider the QoT problem separately from the RWA problem (Tomkos et al. 2004a; Markidis et al. 2007). If the QoTindependently found route is feasible, this is the fastest approach; however, if it does not fit, it is necessary to find a different path. As every single resource along a path per se commonly provides sufficient QoT, it is not clear which link to exclude for subsequent path searches. Common approaches to this problem are to (a) leave out one by one or (b) find several paths in the first step ( $k$-shortest paths) and then check one candidate after the other. The second approach is more efficient only if the time to find $k$ paths in one step is less than the sum of $k$ subsequent 
path searches, weighted each with the likelihood $p(k)$ of finding in $k$ subsequent routings only unfeasible paths. Consequently, for well-designed physical layers and not too degradation sensitive signal formats, the single-path routing with occasional subsequent path searches is economic. To decrease the probability of not finding a feasible path in the first routing attempt, we might adjust the weights used by the routing algorithm to reflect the physical layer's state. This is discussed next.

\subsubsection{Q-Factor-Based Integration of Impairments in Routing Decisions}

A straightforward strategy employed to include physical layer impairments in the routing decisions is to incorporate impairments into the cost function. However, a cost function correctly considering linear and non-linear impairments is still an open question. Different analytical models have been developed to describe reference links (Markidis et al. 2007; Brandt-Pearce et al. 2007). Only few studies consider the simultaneous impact of GVD, DGD, ASE and non-linear phase shift (Ezzahdi et al. 2006). Other more universal metrics have been proposed, including the average measured Q (Deng et al. 2005) and noise variance (Brandt-Pearce et al. 2007). In any case, accurate $\mathrm{Q}$ estimation is a heavy computational task requiring offline calculation. An approach to derive $\mathrm{Q}$ estimates for any path from some end-to-end monitored QoT metrics is outlined next.

\subsubsection{Network Kriging}

In (Chua et al. 2006), a method to predict the performance of paths based on collected information on other paths is presented. It relates to the principle known from applied probability and statistics (Cressie 1993) and is correspondingly called network kriging. In (Sambo et al. 2009a); (b), it is shown that the approach can be applied to predict end-to-end QoT based on monitored end-to-end QoT of other paths. This is achieved by setting up and solving an equation system considering how different paths share resources. The more already monitored paths share each resource of the path to be evaluated, the better the prediction approximates the unknown end-to-end QoT. As only paths that share the same resources contribute to the prediction, it is sufficient to inform only nodes participating in a path on the monitored end-to-end performance via the control plane. Flooding of monitored QoT per path is not required, making the approach scalable. However, to predict the QoT the entire path needs to be known in advance. Therefore, the approach fits best to a scheme where a limited set of candidate paths per ingress-egress node pair is a priori defined. Other than that, the candidate paths are not considered. Seemingly a restriction, this complies with the trend to reduce routing freedom, which can be widely observed in recent proposals and standardisation attempts targeting at more connection oriented IP networks for better traffic engineering options. 


\subsubsection{Ant Colony Optimisation}

Over many years now, ant colony has proven to be a heuristic approach that can be applied successfully for network control and other issues that need to consider a multitude of obviously heterogeneous criteria. Ant colony optimisation is based on ant-like mobile agents that cooperate with each other while randomly exploring possible paths. Based on information collected by each agent and the information left behind at every node by all agents, iteratively decentralised routing information is created. In (Lee 2005; Pavani et al. 2008), a distributed IA-RWA scheme based on ant colony optimisation paradigm is presented. The next hop (outgoing optical channel) is determined based on a preference value (pheromone-level) derived from the information left behind by ants that previously passed. To not end up in a static routing, the preference values fade over time (alike pheromones evaporate) and therefore need to be refreshed from time to time. The rate at which preferences fade determines how many agents are required to keep a consistent routing information available and how fast the routing information adopts to abrupt changes like fibre breaks. This approach is rock solid, but being heuristic, it is difficult to optimise and hard to trust in, even though ants are one of the most successful species on earth.

A similar heuristic exploiting routing history instead of agents is presented in (Marin et al. 2007). Other heuristics applicable to perform IA-RWA include the genetic algorithm as proposed in (Ali et al. 1999; Lima et al. 2003), stimulated annealing (Mukherjee et al. 1996) and taboo search (Yan et al. 2001; Yang et al. 2005). These cannot be implemented or distributed and are computationally exhaustive, but are capable to accurately approach the global optimum with increasing computation effort.

\subsubsection{Multi-Constrained Wavelength Routing (mCBR)}

The major disadvantage of most integrated IA-RWA approaches is that the physical layer impairments need to be reduced to a single scalar cost value. Thereby, the information on individual parameters is lost, and it is necessary to define different cost functions that consider specific sensitivities of different signal formats correctly (also known as reference link models). Considering each physical layer impairment/ constraint and how it changes hop-by-hop individually would be more convenient. Firstly, this allows finding paths that fit constraint by constraint, and secondly, this allows identification of specific weaknesses of certain paths. Especially in case of multi-hop paths, the latter would enable to profitably combine segments that equalise each other.

DWP provides exactly that by replacing the cost value by a vector of constraints and applying lattice algebra (Jukan 2004). Being a multi-constraint approach (mCBR), dynamic programming rules do not fit and therefore, the approach is not scalable and thus is limited to small networks in terms of node, link and wavelength-conversion counts. The DWP scheme suggests maximal spreading of the computing 
effort to (a) split the computation effort to as many processing units as possible and (b) to have real-time access to locally monitored parameters. With DWP, all relevant parameters are summed-up along potential paths by distributing path messages; dropping messages where constraints are not met (branch and bound). Being vectorbased, any number and type of constraint can be considered in parallel; for example, QoT/BER, delay and reliability, as typically specified within SLAs. The fulfilment of constraints is guaranteed by DWP, as long as the parameters applied during path evaluation do not change. To cope with parameter changes, the selection strategy should be to select the path with sufficient headroom for constraints dependent on dynamic parameters. A strategy how to do so, however, is not presented, neither are the implications evaluated.

\subsubsection{ILP-Based IA-RWA}

Today, ILP is commonly applied to perform offline IA-RWA because of its heavy computational complexity. The challenges with ILP are to (a) define an adequate linear optimisation function and (b) formulate all other demands as side constraints. Once done, all these equations are handed over to a software tool and the result pops out. Seemingly, this is simple; however, results also pop out if some side constraints were not considered or incorrectly defined. There is no way to formally check problem sanity and completeness; that needs to be done by the engineer manually. Many proposals to solve IA-RWA using ILP have been published (Yang et al. 2005; Tomkos et al. 2004b; Cugini et al. 2007). They differ in the type and number of constraints considered, the optimisation target, considered routing demands and network scenario. However, ILP appears inadequate for dynamic environments and is unsuited for real-time control. The inevitable offline operation demands synchronised and, more restrictive, considerably delayed changes in compensation adjustments and traffic assignments, a clear contradiction to any dynamic network/traffic management.

Note that to calculate for a given topology, the control loop specifications and the QoT/BER target per network element should be as outlined in Sect. 2.4.1.2. With impairment control and stabilising of network elements, ILP perfectly fits. The Table 2.4 summarizes typical properties of different approaches.

The properties considered in the table are: complexity - meaning the effort required to determine a feasible path; optimality - is the found path optimal in terms of QoT; providence - does the routing metric consider potential future changes of parameters (respectively history); and robustness - how good does the approach per se respond to spontaneous events (e.g. a link failure). Note that some properties depend on implementation and that some shortcomings can be addressed via addon mechanisms (i.e. link failures can be signalled if they do not affect the routing metric) (Table 2.4). 
Table 2.4 Summary of IA-RWA approach's typical properties

\begin{tabular}{lllll}
\hline Approach & Complexity & Optimality & Providence & Robustness \\
\hline Sequ. IA-RWA & Moderate & Poor (first-fit) & No & High \\
Static Q-estim. & Low & Limited & Yes & Low \\
On-line Q-estim. & Moderate & Good & No & High \\
Krieging Q-estim. & High & Limited & Partly & Low \\
Ant colony & Low & Adjustable & Partly & Adjustable \\
mCBR & High & Perfect & No & High \\
ILP & Exhaustive & Perfect & Possibly & High \\
\hline
\end{tabular}

\subsection{Other Demands and Considerations Related to IA-RWA}

For an IA-RWA strategy to be actually implemented, one needs to consider also fundamental aspects like enabling OIM for indirect evaluation of signal quality or enabling direct OPM (Kilper et al. 2004b). In 2004, ITU-T defined a list of OPM parameters that might be used for impairment-aware RWA (ITUG697): (a) residual GVD, (b) total EDFA input and output powers, (c) a channel's optical power budget, (d) OSNR and (e) Q-factor - as an estimator of the overall optical performance. An effective OIM/OPM strategy shall also support the control plane in performing lightpath establishment and rerouting. Proposals on how to integrate physical layer impairments in GMPLS can be found in (Cugini et al. 2008; Cugini et al. 2004). Monitoring of a path's end-to-end QoT can, in addition to providing the basis for improved routing, also contribute to the control of SLA fulfilment and may trigger autonomous rerouting whenever the headroom diminishes below a constraint specific threshold. If rerouting is done obeying the make-before-break strategy, seamless rerouting can support SLA levels else inaccessible.

Per se, the outlined approaches to IA-RWA are not applicable for packet switching due to the complexity that finding feasible paths introduces. Yet, some experts recently proposed to control the network dynamics by limiting routing freedom. For instance, MPLS exactly performs that per flow (per ingress/egress pair). The routing underlying a LSP typically does not change, and thus the effective effort introduced by finding feasible paths to transport a certain flow type inversely depends on the LSP lifetime. Complex set-up procedures for long living LSPs thus actually introduce less effort if thereby frequent rerouting can be evaded.

It should be highlighted that the control loop performance targets for the deployment of physical impairments compensation mechanisms in meshed transmission networks with dynamic traffic assignment cannot be specified independent of traffic characteristic (holding time distribution), traffic matrix (likelihood of flows per node pair) and network topology (average and maximum path length). Even for network architectures comprising restriction to a certain topology and stable traffic distribution, the specs need to be individually derived. Only if QoT stabilisation is reached within a fraction of a single optical pulse or if adjustments have per se no effect on QoT $\left(\mathrm{BER}_{\text {transient }}=0\right)$, traffic assignment dynamics independent QoT could be achieved. Assuming that this is not possible today, the demand on 
stabilisation speed is directly related to traffic assignment dynamics. To achieve the same BER as for non-dynamic optical connections (lines), the target QoT needs to provide sufficient extra headroom. The slower the stabilisation, the more hops, the more dynamic traffic changes actually cause adjustments, the more QoT headroom is effectively required and that needs to be granted by constraint-based RWA.

Finally, a dynamically switched Layer 0 (analogous physical layer) lacking perfect transparent signal regeneration within every hop can, in general, not grant constant QoT and thus shall not be made responsible for end-to-end BER liability.

\section{Conclusions}

Advanced signal processing, management and monitoring techniques are being developed for the next generation of optical networks, which are quickly evolving towards dynamically reconfigurable optical transport networks in which impairment-aware control planes are mandatory in order to grant the required endto-end QoT and QoS.

Simultaneously, a convergence between wide area and access optical networks is taking place. This actual trend in access and metro networks demands, significantly enhance the requirements of signal management and monitoring while maintaining the targets for low cost and passiveness of the network as much as possible. This is especially relevant as signal impairments link losses, Rayleigh Backscattering are significantly increased demanding the development of strategic deployments, monitoring systems and Extender Boxes, as proposed in new recommendations, as G.984.6.

Effective preventive maintenance methods are needed to help network operators reduce the operation-and-maintenance expenses. Most of the monitoring solutions have been based on the well-known OTDR technique. In the framework of the SARDANA project, an elaborated monitoring approach was studied for a converged metro/access network which is a combination of WDM bidirectional rings and TDM access trees. Alternative monitoring approaches based on OFDR and OCDM techniques have also appeared in the scientific literature. However, among many approaches proposed so far, there are no standardised and mature infrastructure monitoring methods that fulfil the operators' needs.

The need for performance monitoring tools (other than a simple BER measurement at line extremity) is reinforced by the evolution of optical transport networks towards larger transmission reach and flexible wavelength paths.

Several optical monitoring techniques have been used for more than one decade. Spectral techniques are well suited to the monitoring of basic parameters (e.g. wavelength value, out-of-band OSNR) of an entire WDM multiplex. Improvements are still required to achieve in-band OSNR measurement of a set of channels with suitable trade-off between measurement accuracy and speed.

Single channel techniques provide a deeper insight into the various optical impairments. Among them, single-tap asynchronous sampling offers many ad- 
vantages, including simplicity, rapidity, high sensitivity to low impairment levels and bit rate independence. Two-tap sampling has been recently proposed and has attractive performances in presence of multiple impairments and complex modulation schemes. These techniques will have to be cost-effective compared to optical channel estimation which becomes available with advanced digital signal processing in the receivers.

$\mathrm{CD}$ monitoring is an important issue to be addressed in reconfigurable WDM optical networks. All-optical CD compensation using RR-based integrated optical filters can be an effective solution for WDM transmission in comparison to other approaches, such as FBG or dispersion-compensating fibres. Recently proposed optical RR filters with internal reflections using Sagnac loops are a promising architecture to achieve periodic and tunable dispersion-compensating modules for DWDM applications in compact and lightweight devices.

The prime targets of impairment control and compensation shall be improving the end-to-end QoT and stabilising fluctuating QoT caused by dynamic resource assignments. With dynamic transmission networks, impairment-aware routing becomes responsible for the provision of optical paths that end-to-end assure a signal-format-dependent QoT sufficient for the QoS requirements posted by the connection requesting services.

\section{References}

10Gb/s Ethernet Passive Optical Network standard, IEEE 802.3av (http://www.ieee802.org/3/av/) Agrawal, G.P.: Nonlinear Fiber Optics, 4th edn. Academic, Boston (2007)

Ahmed, J., Monti, P., Wosinska, L.: Concurrent processing of multiple LSP request bundles on a PCE in a WDM network. In: Proceedings of OSA/IEEE Optical Fiber Communication/National Fiber Optic Engineers Conference OFC/NFOEC 2010, San Diego, Mar 2010

Ali, M., Ramamurthy, B., Deogun, J.S.: Routing algorithms for all-optical networks with power consideration: the unicast case. In: Proceedings of the 8th IEEE ICCCN 1999, pp. 335-340, (1999)

Anderson, T.B., Dods, S.D., Wong, E., Farrell, P.M.: Asynchronous measurement of chromatic dispersion from waveform distortion. Paper OWN4, OFC 2006

Arbab, V.R., Wu, X., Willner, A.E., Weber, C.L.: Optical performance monitoring of data degradation by evaluating the deformation of an asynchronously generated I/Q data constellation. Paper P3.23, ECOC 2009

ITU.: Architecture for the Automatically Switched Optical Network (ASON), ITUTRec.G8090/Y.1304, Nov 2001

IETF.: A Path Computation Element (PCE)-Based Architecture, IETF RFC 4655, Aug 2006

Banerjee, A., Drake, J., Lang, J., et al. Generalized multiprotocol label switching: An overview of signaling enhancements and recovery techniques. IEEE Commun. Mag. 144-151. (2001) (citeseer.ist.psu.edu/banerjee01generalized.html)

Baveja, P.P., Maywar, D.N., Agrawal, G.P.: Optimization of all-optical 2R regenerators operating at $40 \mathrm{~Gb} / \mathrm{s}$ : role of dispersion. J. Lightwave Technol. 27, 3831-3836 (2009)

Bellman, R.E.: Dynamic Programming. Princeton University Press. Republished 2003, Dover, ISBN 978-0486428093 (1957) 
Bendelli, G., Cavazzoni, C., Girardi, R., Lano, R.: Optical performance monitoring techniques. In: Proceedings of 26th European Conference on Optical Communication (ECOC), vol. 4, 3-7 September, Munich, Germany, pp. 113-116 (2000)

Bertsekas, D.P.: Dynamic Programming and Optimal Control, 3rd edn. Athena Scientific, Belmont (2005). ISBN 978-1886529083

Bocci, M., Bryant, S., Frost, D., Levrau, L., Berger, L.: A framework for MPLS in transport networks, draft-ietf-mpls-tp-framework-12. IETF MPLS Working Group, Nov 2010

Bock, C., Lazaro, J.A., Prat, J.: Extension of TDM-PON standards to a single-fiber ring access network featuring resilience and service overlay. IEEE/OSA J. Lightwave Technol. 2007, 14161421 (2007)

Brandt-Pearce, J.H., Pointurier, M., Subramaniam, Y.: QoT-aware routing in impairmentconstrained optical networks. Proc. GLOBECOM 2007, 26-30 (2007)

Cahill, M., Bartolini, G., Lourie, M., Domash, L.: Tunable thin film filters for intelligent WDM networks. In: Ellison, M.J. (ed.) Proceedings of SPIE, vol. 6286, Advances in Thin Film Coatings for Optical Applications III, Aug 2006

Castoldi, P., Cugini, F., Valcarenghi, L., Sambo, N., Le Rouzic, E., Poirrier, M.J., Adriolli, N., Paolucci, F., Giorgetti, A.: Centralized vs. distributed approaches for encompassing physical impairments in transparent optical networks, Lecture Notes in Computer Science Optical Network Design and Modeling 4534/2007 (2007) 68-77

Caucheteur, C., Mussot, A., Bette, S., Kudlinski, A., Douay, M., Louvergneaux, E., Mégret, P., Taki, M., Gonzalez-Herraez, M.: All-fiber tunable optical delay line. Opt. Express 18, 3093$3100(2010)$

Chen, L.K., Cheung, M.H., Chan, C.K.: From optical performance monitoring to optical network management: research progress and challenges. Proceedings of ICOCN 2004, (2004)

Chen, W., Tucker, R.S., Yi, X., Shieh, W., Evans, J.S.: Optical signal-to-noise ratio monitoring using uncorrelated beat noise. IEEE Photon. Technol. Lett. 17, 2484-2486 (2005)

Chlamtac, I., Ganz, A., Karmi, G.: Lightpath communications: an approach to high-bandwidth optical WAN's. IEEE Trans. Commun. 40, 1171-1182 (1992)

Choi, E., Na, J., Ryu, S., Mudhana, G., Lee, B.: All-fiber variable optical delay line for applications in optical coherence tomography: feasibility study for a novel delay line. Opt. Express 13, 1334-1345 (2005)

Chua, D.B., Kolaczyk, E.D., Crovella, M.: Network kriging. IEEE J. Sel. Areas Commun. 24(12), 2263-2272 (2006)

Chung, Y.C.: Optical performance monitoring techniques; current status and future challenges, ECOC-2008, invited paper. Bruxselles, 21-25 Sept 2008

Cressie, N.A.C.: Statistics for Spatial Data Wiley Series in Probability and Mathematical Statistics: Applied Probability and Statistics. Wiley, New York (1993)

Cugini, F., Andriolli, N., Valcarenghi, L., Castoldi, P.: A novel signaling approach to encompass physical impairments in GMPLS networks. In: Proceedings of IEEE GLOBECOM 2004 Workshops, Dallas, November 29 - December 3 Dallas, Texas, USA, pp. 369-373 (2004)

Cugini, F., Andriolli, N., Valcarenghi, L., Castoldi, P.: Physical impairment aware signaling for dynamic lightpath set up. In: Proceedings of ECOC2005, Glasgow, vol. 4, pp. 979-980. Sept 2005

Cugini, F., Paolucci, F., Valcarenghi, L., Castoldi, P.: Implementing a Path Computation Element (PCE) to encompass physical impairments in transparent networks. In: Proceedings of OFC/NFOEC 2007, 25-29 March, Anaheim, California, USA, pp. 1-3 (2007)

Cugini, F., Sambo, N., Andriolli, N., Giorgetti, A., Valcarenghi, L., Castoldi, P., Le Rouzic, E., Poirrier, J.: Enhancing GMPLS signaling protocol for encompassing quality of transmission (QoT) in all-optical networks. J. Lightwave Technol. 26(19), 3318-3328 (2008)

Deng, T., Subramaniam, S.: Adaptive QoS routing in dynamic wavelength-routed optical networks. Proceedings of BROADNETS 2005, vol. 1, pp. 184-193, (2005)

Dilwali, S., Soundra Pandian, G.: Pulse response of a fiber dispersion equalizing scheme based on an optical resonator. IEEE Photon. Technol. Lett. 4(8), 942-944 (1992) 
Dods, S.D., Anderson, T.B.: Optical performance monitoring technique using delay tap asynchronous waveform sampling. Optical Fiber Communication Conference, Paper OthP5, OFC 2006

Effenberger, F., Meng, S.: In-band optical frequency domain reflectometry in pons. In: OFC/NFOEC, pp. 1-3. (2008)

Ezzahdi, M., Zahr, S., Koubaa, M., Puech, N., Gagnaire, M.: LERP: a quality of transmission dependent heuristic for routing and wavelength assignment in hybrid WDM networks. Proc. ICCCN 2006, 125-136 (2006)

Frigo, N.J., et al.: Centralized in-service OTDR testing in a CWDM business access network. IEEE J. Lightwave Technol. 22(11), 2641-2652 (2004)

$\mathrm{Fu}$, L.B., Rochette, M., Ta'eed, V.G., Moss, D.J., Eggleton, B.J.: Investigation of self-phase modulation based optical regeneration in single mode As $2 \mathrm{Se} 3$ chalcogenide glass fiber. Opt. Express 13, 7637-7644 (2005)

G.7713.1/Y.1704.1, DCM signalling mechanism using PNNI/Q.2931

G.7713.2/Y.1704.2, DCM signalling mechanism using GMPLS RSVP-TE

G.8080/Y.1304, Architecture for the automatically switched optical network

Generalized Multi-Protocol Label Switching (GMPLS) Architecture, IETF RFC 3945, Oct 2004

Gnauck, A.H., Cimini Jr., L.J., Stone, J., et al.: Optical equalization of fiber chromatic dispersion in a 5-Gb/s transmission system. IEEE Photon. Technol. Lett. 2(8), 585-587 (1990)

Halabi, S.: Metro Ethernet. Cisco Press, Indianapolis (2003)

Huang, Y., Heritage, J.P., Mukherjee, B.: Connection provisioning with transmission impairment consideration in optical WDM networks with high-speed channels. J. Lightwave Technol. 23(3), 982-983 (2005)

Hult, J.: A fourth-order Runge-kutta in the interaction picture method for simulating supercontinuum generation in optical fibers. J. Lightwave Technol. 25, 3770-3775 (2007)

IEEE 802.1Qay: Provider Backbone Bridging Traffic Engineering, work in progress, http://www. ieee $802.0 \mathrm{rg} / 1 /$ pages/802.1ay.html.

ITU-T G.697 Optical Monitoring for DWDM Systems, Nov 2009 - Prepublished

ITU-T Recommendation X.200: Information Technology - Opens Systems Interconnection Basic Reference Model: The Basic Model, (1994)

ITU-T G984.6, GPON Optical Reach extension

Jargon, J.A., Wu, X., Willner, A.E.: Optical performance monitoring by use of artificial neural networks trained with parameters derived from delay-tap asynchronous sampling. Paper OThH1, OFC 2009

Jukan, A., Franzl, G.: Path selection methods with multiple constraints in service-guaranteed WDM networks. IEEE/ACM Trans. Netw. 12(1), 59-72 (2004)

Kashyap, R., Chernikov, S.V., McKee, P.F., Williams, D.L., Taylor, J.R.: Demonstration of dispersion compensation in all-fibre photoinduced chirped gratings. Pure Appl. Opt. 4, 425429 (1995)

Kilper, D.C., Bach, R., Blumenthal, D., Einstein, D., Landolsi, T., Ostar, L., Preiss, M., Willner, A.E.: Optical performance monitoring. J. Lightwave Technol. 22, 294-304 (2004a)

Kilper, D.C., Bach, R., Blumenthal, D.J., Einstein, D., Landolsi, T., Ostar, L., Preiss, M., Willner, A.E.: Optical performance monitoring. IEEE/OSA J. Lightwave Technol. 22(1), 294304 (2004b)

Kilper, D.C., Fergunson, A., O'Sullovan, B., Korotky, S.K.: Impact of topology and traffic on physical layer monitoring in transparent networks, OFC, invited paper, (2009)

Kirstaedter, A., Wrage, M., Goeger, G., Fishler, W., Splinner, B.: Current aspects of optical performance monitoring and failure root cause analysis in optical WDM networks. Proceeding SPIE, vol. 5625, 362-373, (2005)

Kompella, K., Rekhter, Y.: Label Switched Paths (LSP) Hierarchy with Generalized MultiProtocol Label Switching (GMPLS) Traffic Engineering (TE) (proposed standard track). IETF RFC4206. (2005) 
Laming, R.I., Robinson, N., Scrivener, P.L., Zervas, M.N., Barcelos, S., Reekie, L.: Dispersion tunable grating in a 10-Gb/s 100-220 km step-index fiber link. IEEE Photon. Technol. Lett. 8, 428-430 (1996)

Lazaro, J.A., Bock, C., Polo, V., Martinez, R.I., Prat, J.: Remotely amplified combined ring-tree dense access network architecture using reflective RSOA-based ONU. OSA J. Opt. Netw. 6(6), 801-807 (2007a)

Lazaro, J.A., Arellano, C., Polo, V., Prat, J.: Rayleigh scattering reduction by means of optical frequency dithering in passive optical networks with remotely seeded ONUs. IEEE Photon. Technol. Lett. 19, 64-66 (2007b)

Lazaro, J.A., Prat, J., Chanclou, P., Tosi Beleffi, G.M., Teixeira, A., Tomkos, I., Soila, R., Koratzinos, V.: Scalable Extended Reach PON, OFC/NFOEC 2008, invited paper OThL2 (2008)

Lee, K.I., Shayman, M.: Optical network design with optical constraints in multi-hop WDM mesh networks. IEICE Transactions on Communications, (2005)

Lee, J.H., Choi, H.Y., Shin, S.K., Chung, Y.C.: A review of the polarization-nulling technique for monitoring optical-signal-to-noise ratio in dynamic WDM networks. IEEE/OSA J. Lightwave Technol. 24(11), 4162-4171 (2006)

Lenz, G., Madsen, C.K.: General optical all-pass filter structures for dispersion control in WDM systems. J. Lightwave Technol. 17(7), 1249-1254 (1999)

Lima, M.A.C., Cesar, A.C., Araujo, A.F.R.: Optical network optimization with transmission impairments based on genetic algorithm. In: Proceedings of the SBMO/IEEE IMOC, vol. 1, pp. 361-365, (2003)

Liu, X., Kao, Y.-H.: A simple OSNR monitoring technique independent of PMD and chromatic dispersion based on a 1-bit delay interferometer. In: Proceedings of European Conference on Optical Communication, PaperMo4.4.5, Cannes, (2006)

Lizé, Y.K., Yang, J.-Y., Christen, L.C., Wu, X.-X., Nuccio, S., Wu, T., Willner, A.E., Kashyap, R., Séguin, F.: Simultaneous and independent monitoring of OSNR, chromatic and polarization mode dispersion for NRZ-OOK, DPSK and Duobinary. In: Proceedings of Optical Fiber Communication Conference, Paper OThN2, Anaheim, Mar 2007

Lopez, E.T., Lazaro, J.A., Arellano, C., Polo, V., Prat, J.: Optimization of rayleigh-limited WDMPONs with reflective ONU by MUX positioning and optimal ONU gain. IEEE Photon. Technol. Lett. 22, 97-99 (2010)

Luis, R., Andre, P., Teixeira, A., Monteiro, P.: Performance monitoring in optical networks using asynchronously acquired samples with non ideal sampling systems and intersymbol interference. J. Lightwave Technol. 2(11), 2452-2459 (2004)

Madsen, C.K., Lenz, G.: Optical all-pass filters for phase response design with applications for dispersion compensation. IEEE Photon. Technol. Lett. 10, 994-996 (1998)

Madsen, C.K., Zhao, J.H.: A general planar waveguide autoregressive optical filter. J. Lightwave Technol. 14(3), 437-447 (1996)

Madsen, C.K., Lenz, G., Bruce, A.J., et al.: Multistage dispersion compensator using ring resonators. Opt. Lett. 24(22), 1555-1557 (1999)

Madsen, C.K., Walker, J.A., Ford, J.E., et al.: A tunable dispersion-compensating MEMS all-pass filter. IEEE Photon. Technol. Lett. 12(6), 651-653 (2000)

Mamyshev, P.V.: All-optical data regeneration based on self-phase modulation effect. In: Proceedings of ECOC, 475-476 (1998)

Mannie, E., Papadimitriou, D.: Generalized Multi-Protocol Label Switching (GMPLS) Extensions for Synchronous Optical Network (SONET) and Synchronous Digital Hierarchy (SDH) Control (standards track), IETF RFC3946. (2004)

Marin, E., Sánchez, S., Masip, X., Solé, J., Maier, G., Erangoli, W., Santoni, S., Quagliotti, M.: Applying prediction concepts to routing on semi transparent optical transport networks. In: Proceedings of ICTON 2007, pp. 32-36, (2007)

Markidis, G., Sygletos, S., Tzanakaki, A., Tomkos, I.: Impairment aware based routing and wavelength assignment in transparent long Haul networks. Optical network design and 
monitoring. In: Optical Network Design and Modeling. Lecture Notes in Computer Science, pp. 48-57. Springer, Berlin/Heidelberg (2007)

Martinez, R., Pinart, C., Cugini, F., Andriolli, N., Valcarenghi, L., Castoldi, P., Wosinska, L., Comellas, J., Junyent, G.: Challenges and requirements for introducing impairment-awareness into the management and control planes of ASON-GMPLS WDM networks. IEEE Commun. Mag. 44(12), 76-85 (2006)

Militello, M., et al.: Optical dynamic monitoring in next generation networks. IEEE CONTEL Conference, vol. ISBN 978-953-184-130-6, pp. 289-291, Zagreb, June 2009

Montalvo, J.: Applications of ring resonators and fiber delay lines for sensors and WDM networks. Ph.D. thesis, Univ. Carlos III Madrid, 2008

Mueller, K., Hanik, N., Gladish, A., Foisel, H-M., Caspar, C.: Application of amplitude histograms for quality of service measurements of optical channels and fault identification, ECOC 98, pp. 707-708

Mukherjee, B., Ramamurthy, S., Banerjee, D., Mukherjee, A.: Some principles for designing a wide-area WDM optical network. IEEE/ACM Trans. Netw. 4(5), 684-696 (1996)

Nguyen, T.N., Gay, M., Bramerie, L., Chartier, T., Simon, J.-C.: Noise reduction in 2R-regeneration technique utilizing self-phase modulation and filtering. Opt. Express 5, 1737-1747 (2006)

Omella, M., Lazaro, J.A., Polo, V., Prat, J.: Driving requirements for wavelength shifting in colorless ONU with dual-arm modulator. J. Lightwave Technol. 27(17), 3912-3918 (2009a). ISSN: 0733-8724

Omella, M., Papagiannakis, I., Schrenk, B., Klonidis, D., Lazaro, J.A., Birbas, A.N., Kikidis, J., Prat, J., Tomkos, I.: $10 \mathrm{~Gb} / \mathrm{s}$ full-duplex bidirectional transmission with RSOA-based ONU using detuned optical filtering and decision feedback equalization. Opt. Express 17(7), 50085013 (2009b)

Orta, R., Savi, P., Tascone, R., Trinchero, D.: Synthesis of multiple-ring-resonator filters for optical systems. IEEE Photon. Technol. Lett. 7(12), 1447-1449 (1995)

Othonos, A., Kalli, K.: Fiber Bragg Gratings: Fundamentals and Applications in Telecommunications and Sensing. Artech House, Norwood (1999)

Ouelette, F.: Dispersion cancellation using linearly chirped Bragg grating filters in optical waveguides. Opt. Lett. 12, 847-849 (1987)

Pachnicke, S., Luck, N., Krummrich, P.M.: Online Physical-Layer Impairment-Aware Routing with Quality of Transmission Constraints in Translucent Optical Networks, ICTON 2009 Tu.A3.5

Pan, Z., Yu, C., Willner, A.E.: Optical performance monitoring for the next optical communication network. Opt. Fiber Technol. 16, 20-45 (2010)

Park, K.J., Youn, C.J., Lee, J.H., et al.: Performance comparisons of chromatic dispersionmonitoring techniques using pilot tones. IEEE Photon. Technol. Lett. 15, 873-875 (2003)

Pavani, G.S., Zuliani, L.G., Waldman, H., Magalhães, M.F.: Distributed approaches for impairment-aware routing and wavelength assignment algorithms in GMPLS networks. Comput. Netw. 52(10), 1905-1915 (2008)

Petersen, M.N., Pan, Z., Lee, S., et al.: Online chromatic dispersion monitoring and compensation using a single inband subcarrier tone. IEEE Photon. Technol. Lett. 14, 570-572 (2002)

Pinart, C., Amrani, A., Junyent, G.: Design and experimental implementation of a hybrid optical performance monitoring system for in-service SLA guarantee. 9th IFIP/IEEE International Symposium on Integrated Network Management (IM 2005), Nice, 16-19 May 2005

Prat, J., Lazaro, J.A., Chanclou, P., Cascelli, S.: Passive OADM Network Element for Hybrid RingTree WDM/TDM-PON, ECOC'09, Proceedings, paper: P6.23, Vienna, Sept 20-24, 2009

Provost, L., Finot, C., Mukasa, K., Petropoulos, P., Richardson, D.J.: Design scaling rules for 2Roptical self-phase modulation-based regenerators. Opt. Express 15, 5100-5112 (2007a)

Provost, L., Finot, C., Mukasa, K., Petropoulos, P., Richardson, D.J.: Generalisation and experimental validation of design rules for self-phase modulation-based 2R-regenerators. In: OFC/NFOEC (2007b)

Provost, L., Parmigiani, F., Petropoulos, P., Richardson, D.J.: Investigation of timing jitter reduction in a bidirectional 2R all-optical Mamyshev regenerator. In: OFC/NFOEC (2008) 
RFC 4202 - Routing Extensions for GMPLS. Oct 2005 (Standards Track)

RFC 4204 - Link Management Protocol (Standards Track)

RFC 4974 - Generalized MPLS (GMPLS) RSVP-TE Signaling Extensions in Support of Calls

Rossi, G., Dimmick, T.E., Blumenthal, D.J.: Optical performance monitoring in reconfigurable WDM optical networks using subcarrier multiplexing. IEEE/OSA J. Lightwave Technol. 18, 1639-1648 (2000)

Rotwitt, R., Guy, M.J., Boscovik, A., Noske, D.U., Taylor, J.R., Kashyap, R.: Interaction of uniform phase picoseconds pulses with chirped and unchirped photosensitive fibre Bragg gratings. Electron. Lett. 30, 995-996 (1991)

Saleh, M., Simmons, J.M.: Evolution toward the next-generation core optical network. IEEE/OSA J. Lightwave Technol. 24(9), 3303-3321 (2006)

Salvadori, E., Ye, Y., Zanardi, A., Woesner, H., Carcagni, M., Galimberti, G., Martinelli, G., Tanzi, A., LaFauci, D.: A study of connection management approaches for an impairmentaware optical control plane. In: Proceedings of IFIP ONDM2007. Lecture Notes in Computer Science, vol. 4534, pp. 229-238. Springer, Athens (2007a)

Salvadori, E., Ye, Y., Zanardi, A., Woesner, H., Carcagni, M., Galimberti, G., Martinelli, G., Tanzi, A., LaFauci, D.: Signalling-based architectures for impairment-aware lightpath set-up in GMPLS networks. In: Proceedings of IEEE GLOBECOM 2007, Washington, pp. 2263-2268. Nov $2007 \mathrm{~b}$

Sambo, N., Giorgetti, A., Andriolli, N., Cugini, F., Valcarenghi, L., Castoldi, P.: GMPLS signaling feedback for encompassing physical impairments in transparent optical networks. In: Proceedings of IEEE GLOBECOM2006, Sanfrancisco, pp. 1-5. Nov 2006

Sambo, N., Pointurier, Y., Cugini, F., Castoldi, P., Tomkos, I.: Lightpath establishment in PCEbased dynamic transparent optical networks assisted by end-to-end quality of transmission estimation. In: Proceedings of ICTON 2009, Mo.D3.1, (2009a)

Sambo, N., Pointurier, Y., Cugini, F., Valcarenghi, L., Castoldi, P., Tomkos, I.: Lightpath establishment in distributed transparent dynamic optical networks using network kriging. ECOC 2009 proceedings, paper 1.5.3, (2009b)

Shake, I., Takara, H.: Averaged Q-factor method using amplitude histogram evaluation for transparent monitoring of optical signal-to-noise ratio degradation in optical transmission system. J. Lightwave Technol. 20, 1367-1373 (2002)

Shake, I., Takara, H.: Chromatic dispersion dependence of asynchronous amplitude histogram evaluation of NRZ signal. J. Lightwave Technol. 21(10), 2154-2161 (2003)

Shake, I., Takara, W., Kawanishi, S., Yamabayashi, Y.: Optical signal quality monitoring method based on optical sampling. Electron. Lett. 34(22), 2152-2154 (1998)

Shake, I., Takara, H., Kawanishi, S.: Simple measurement of eye diagram and BER using highspeed asynchronous sampling. J. Lightwave Technol. 22, 1296-1302 (2004)

Shieh, W., Tucker, R.S., Chen, W., Yi, X., Pendock, G.: Optical performance monitoring in coherent optical OFDM systems. Opt. Express 15(2), 350-356 (2007)

Strand, J., Chiu, A.L., Tkach, R.: Issues for routing in the optical layer. IEEE Commun. Mag. 39(2), 81-87 (2001)

Striegler, A.G., Schmauss, B.: Analysis and optimisation of SPM-Based 2R signal regeneration at 40 Gb/s. J. Lightwave Technol. 24, 2835-2842 (2006)

Takiguchi, K., Okamoto, K., Moriwaki, K.: Dispersion compensation using a planar lightwave circuit optical equalizer. IEEE Photon. Technol. Lett. 6(4), 561-564 (1994)

Teixeira, A., Costa, L., Frantzl, G., Azodolmolky, S., Tomkos, I., Vlachos, K., Zsigmond, S., Cinkler, T., Tosi Beleffi, G., Gravey, P., Loukina, T., Lázaro, J.A., Vazquez, C., Montalvo, J., Le Rouzic, E.: An integrated view on monitoring and compensation for dynamic optical networks from management to physical layer. Photon. Netw. Commun. (2009). doi:DOI: 10.1007/s11107-008-0183-5

Tomkos, I.: Transport performance of WDM metropolitan area transparent optical networks. In: Proceedings of OFC, Mar 2002, pp. 350-352 
Tomkos, I., Vogiatzis, D., Mas, C., Zacharopoulos, I., Tzanakaki, A., Varvarigos, E.: Performance engineering of Metropolitan area optical networks through impairment constraint routing. IEEE Optical Communications Magazine, pp. 40-47, (2004a)

Tomkos, I., Vogiatzis, D., Mas, C., Zacharopoulos, I., Tzanakaki, A., Varvarigos, E.: Performance engineering of metropolitan area optical networks through impairment constraint routing. IEEE Commun. Mag. 42(8), S40-S47 (2004b)

Tsuritani, T., Miyazawa, M., Kashihara, S., Otani, T.: Optical path computation element interworking with network management system for transparent mesh networks. In: Proceedings of OFC/NFOEC 2008. pp. 1-10. San Diego, Mar (2006)

Valenti, A., Bolletta, P., Pompei, S., Matera, F.: Experimental investigations on restoration techniques in a wide area Gigabit ethernet optical test-bed based on Virtual Private LAN Service. In: Proceedings of ICTON 09, Ponta Delgada, (2009)

Vargas, S., Vázquez, C.: Synthesis of optical filters using Sagnac interferometer in ring resonator. IEEE Photon. Technol. Lett. 19, 1877-1879 (2007)

Vargas, S., Vázquez, C., Pena, J.M.S.: Novel tunable optical filter employing a fiber loop mirror for synthesis applications in WDM. 14th Annual meeting LEOS, 2:899-900, 2001

Vargas, S., Vázquez, C.: Synthesis of optical filters using microring resonators with ultra-large FSR. Opt. Express 18, 25936-25949 (2010)

Wu, X., Jargon, J.A., Jia, Z., Paraschis, L., Skoog, R.A., Willner, A.E.: Optical performance monitoring of PSK data channels using artificial neural networks trained with parameters derived from delay-tap asynchronous diagrams via balanced detection. Paper P3.04, ECOC 2009

Wuilmart, L., et al.: A PC-based Method for the localisation and quantisation of faults in passive tree-structured optical networks using OTDR technique. In: Proceedings of IEEE LEOS'96, Boston, pp. 122-123, Nov 1996

Yan, S., Ali, M., Deogun, J.: Route optimization of multicast sessions in sparse light-splitting optical networks. IEEE GLOBECOM 2001, vol. 4, pp. 2134-2138, (2001)

Yang, X., Shen, L., Ramamurthy, B.: Survivable lightpath provisioning in WDM mesh networks under shared path protection and signal quality constraints. IEEE/OSA J. Lightwave Technol. 23(4) (2005). pp. 1556

Yüksel, K., Moeyaert, V., Wuilpart, M., Mégret, P.: Optical layer monitoring in Passive Optical Networks (PONs): a review, International Conference on Transparent Optical Networks (ICTON), paper Tu.B1.1, Athens, 22/06-26/06, 2008

Yuksel, K., Wuilpart, M., Moeyaert, V., Mégret, P.: A novel monitoring technique for passive optical networks based on optical frequency domain reflectometry and fiber Bragg gratings, ICTON 2010, paper ThA2.2, 27 June - 1 July, Munich

Zang, H., Jue, J.P., Mukherjee, B.: A review of routing and wavelength assignment approaches for wavelength-routed optical WDM networks. SPIE/Baltzer Opt. Netw. Mag. 1(1), 47-60 (2000)

Zou, N., Namihira, Y., Ndiaye, C., Ito, H.: Fault location for branched optical fiber networks based on ofdr technique using fsf laser as light source OFC/NFOEC, p. NWC2, (2007) 